\title{
ZUSES
}

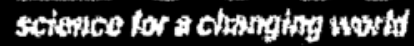

\section{CHEMICAL COMPOSITION OF WEATHERED AND LESS WEATHERED STRATA OF THE MEADE PEAK PHOSPHATIC SHALE MEMBER OF THE PERMIAN PHOSPHORIA FORMATION B. Measured Sections C and D, Dry Valley, Caribou County, Idaho}

by J. R. Herring ${ }^{1}$, S. A. Wilson ${ }^{1}$, L. A. Stillings ${ }^{2}$, A. C. Knudsen ${ }^{3}$ M. E. Gunter ${ }^{3}$, R. G. Tysdal ${ }^{1}$, R. I. Grauch ${ }^{1}$, G. A. Desborough ${ }^{1}$, and R.A. Zielinski ${ }^{1}$

Open-File Report 99-147-B

paper edition

2000

Prepared in Cooperation With

U.S. Bureau of Land Management

U.S. Forest Service

Agrium U.S. Inc.

FMC Corporation

J.R. Simplot Company

Rhodia Inc.

Solutia Inc.

This report is preliminary and has not been reviewed for conformity with U.S. Geological Survey editorial standards or with the North American Stratigraphic Code. Any use of trade, firm, or product names is for descriptive purposes only and does not imply endorsement by the U.S. Government.

\section{U. S. DEPARTMENT OF THE INTERIOR \\ U. S. GEOLOGICAL SURVEY}

${ }^{1}$ U.S. Geological Survey, DFC, Box 25046, MS 973, Denver, CO 80225

${ }^{2}$ U.S. Geological Survey, MacKay School Of Mines, MS 176, Reno, NV 89557-004

${ }^{3}$ Univ. of Idaho, Moscow, ID 838443022 
$\begin{array}{lr}\text { ABSTRACT } & 3\end{array}$

INTRODUCTION 4

Background 4

Location and General Geology 4

$\begin{array}{lc}\text { Correlation with Measured Sections } & 6\end{array}$

$\begin{array}{lr}\text { METHODS } & 6\end{array}$

Field Sampling $\quad 6$

$\begin{array}{ll}\text { Rock Sample Preparation } & 7\end{array}$

$\begin{array}{ll}\text { Analysis } & 7\end{array}$

$\begin{array}{lr}\text { RESULTS } & 10\end{array}$

$\begin{array}{ll}\text { ACKNOWLEDGMENTS } & 11\end{array}$

REFERENCES CITED 12

FIGURES

Figure 1. Index map of southeastern Idaho showing location of measured sections from which samples were collected.

Figure 2. Comparison of measured uranium concentrations by delayed neutron analysis in channel samples with gamma-ray spectroscopy measurements taken at 1-foot true-thickness stations through the same intervals and arithmetically averaged.

Figure 3. Concentrations of various elements in Section C and D. The concentrations of some trace elements are scaled logarithmically to enhance visible differences at their lower concentrations. Major lithologic units (lower ore, middle waste, upper ore, and upper waste) are shown as zones set off by dashes.

\section{TABLES}

Table 1. Concentrations of major, minor, and trace elements for individual samples and replicated samples for Measured Section C.

Table 2. Concentrations of major, minor, and trace elements for individual samples and replicated samples for Measured Section D.

Table 3. Accuracy and precision-concentrations of major, minor, and trace elements in Standard Reference Materials and in duplicate samples accompanying analysis of samples from Measured Section C and D. 


\begin{abstract}
This study reports bulk chemical composition of rocks collected from two exposed, measured stratigraphic sections at a phosphate mine in southeastern Idaho. The samples constitute a set of channel-sampled intervals across the entire thickness of the Meade Peak Phosphatic Shale Member of the Phosphoria Formation at two locations exposed during mining. The samples characterize the lower phosphate ore, interlayered middle waste rock, upper ore, and upper waste units of the member. The rocks from measured section $C$ lie closer to the original, pre-mined ground surface than those of measured section D and, hence, are more oxidized and weathered than those of Section D. Section C includes a channel sample of the overlying Rex Chert Member, and Section D includes a channel sample of the underlying Grandeur dolomite, with both samples taken immediately adjacent to the Meade Peak Member.
\end{abstract}




\section{INTRODUCTION}

\section{Background}

The U.S. Geological Survey (USGS) has studied the Permian Phosphoria Formation in southeastern Idaho and the Western U.S. Phosphate Field throughout much of the twentieth century. In response to a request by the U.S. Bureau of Land Management (BLM), a new series of resource and geoenvironmental studies was initiated by the USGS in 1998. Present studies consist of (1) integrated, multidisciplinary research directed toward resource and reserve estimations of phosphate in selected 7.5-minute quadrangles; (2) elemental residence, mineralogical and petrochemical characteristics; (3) mobilization and reaction pathways, transport, and disposition of potentially toxic elements associated with the occurrence, development, and use of phosphate; (4) geophysical signatures; and (5) improving the understanding of depositional origin. To carry out these studies, the USGS has formed cooperative research relationships with two Federal agencies, BLM and the U.S. Forest Service (USFS), which are responsible for land management and resource conservation on public lands; and with five private companies currently leasing or developing phosphate resources in southeastern Idaho. The companies are Agrium U.S. Inc. (Rasmussen Ridge mine), FMC Corporation (Dry Valley mine), Rhodia Inc. (Wooley Valley mine-inactive), J.R. Simplot Company (Smoky Canyon mine), and Solutia Inc. (Enoch Valley mine). Because raw data acquired during the project will require time to interpret, the data are released in open-file reports for prompt availability to other workers. The open-file reports associated with this series of resource and geoenvironmental studies are submitted to each of the Federal and industry cooperators for technical review; however, the USGS is solely responsible for the data contained in the reports.

\section{Location and General Geology}

The location of the measured sections is shown in figure 1 . The sections lie approximately $25 \mathrm{~km}$ northeast of Soda Springs, Idaho, in an area of southeastern Idaho that has had extensive phosphate mining over the past several decades and currently has four active phosphate mines. Service (1966) provides an evaluation of the western phosphate industry in Idaho and a brief description of the mining history, ore occurrence, and geology. More detailed discussion of the Phosphoria Formation in the Western Phosphate Field is given by McKelvey and others (1959). Cressman and Swanson (1964) discuss detailed stratigraphy and petrology of these same rock units in nearby southwestern Montana. Gulbrandsen and Krier (1980) discuss general aspects of the large and rich phosphorus resources in the Phosphoria Formation in the vicinity of the Soda Springs, Idaho. Gulbrandsen (1966, 1975, and 1979) summarizes bulk chemical compositional data for various lithologies of the phosphatic intervals in the Phosphoria Formation. 


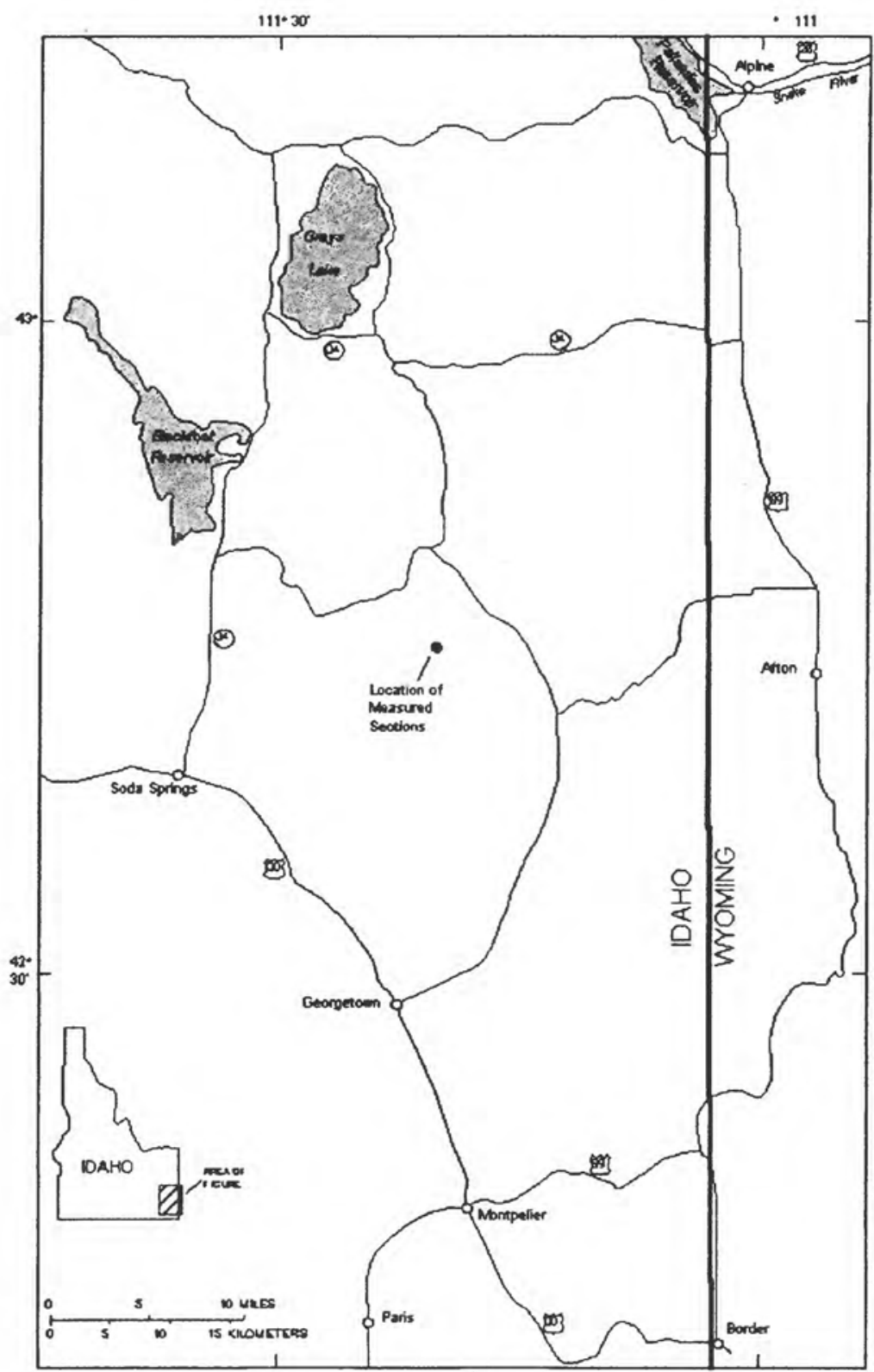

Figure 1. Index map of southeastern Idaho showing location of measured sections from which samples were collected. 


\section{Correlation with Measured Sections}

Stratigraphic sections of the Phosphoria Formation were measured and sampled by the USGS at the Dry Valley mine in southeastern Idaho. Brief descriptions of the stratigraphic sections from which the samples discussed in this report have been taken are already published (Tysdal and others, 2000), although no thin section, X-ray, or analytical technique other than gamma-ray spectrometry has been used to augment the field descriptions of the rock units of that report. This report lists the analytical information for rock sequences described from those sections. The two reports are best used together in a complementary fashion to obtain both descriptive and analytical information about the rock sections. Informal bed names - introduced by Hale (1967, p. 152) and used generally throughout southeastern Idaho-are included in a column of each of the data tables in this report and in accompanying figures that graphically display the analytical data. Informal bed names used only within a specific mine are not presented here. English units of measurement are used throughout this report to facilitate direct correspondence with units in the extensive historical literature on the Phosphoria and with current industry usage. Thicknesses and interval boundary footages listed here are true thickness of the strata at the sample site; these thicknesses are corrected for apparent thickening due to dip of the strata at the exposed sections at the mine benches.

The Phosphoria Formation in the vicinity of the measured sections consists of three members, which in ascending order are the Meade Peak Phosphatic Shale, the Rex Chert, and the informally named cherty shale (McKelvey and others, 1959; Hale, 1967; Rioux and others, 1975). The measured sections of this report focus on the Meade Peak Phosphatic Shale Member. The Meade Peak unconformably overlies the Grandeur Tongue of the Permian Park City Formation, and the cherty shale member is overlain by the Triassic Dinwoody Formation.

Both sections were measured at essentially the same geographic position, but section wpsC (western phosphate section $\mathrm{C}$ ) was of rock about $150 \mathrm{ft}$ higher in elevation than that of section wpsD, and much closer to the land surface that existed just prior to mining. Measuring a pair of sections close together, but at different depths below this land surface, permits evaluation of important effects of weathering on rock geochemistry. Except for upper waste strata of wpsD, the two sections were measured on horizontal surfaces exposed by mining equipment. The two sections are of similar thickness, as expected. The upper ore zone of section wpsD was not exposed on the floor of the open-pit mine because it was mostly covered by talus and waste-pile debris at the base of the pit wall. Waste above the upper ore zone of wpsD was measured on the pit wall.

\section{METHODS}

\section{Field Sampling}

The samples within the measured sections that were obtained for geochemical and petrological analysis were taken as channeled samples across the entire thickness of the interval, as noted in the data tables. The choice of sampling intervals is intended to characterize strata of more or less uniform lithology and of a broad thickness that can be handled by typical mine equipment should the results of our analyses suggest that separate handling of such zones would be advantageous. Within these broad intervals, we have sampled finer-scale strata, sometimes as little as one foot thick, where we have noted a lithology different or distinctive from the broad interval as a whole. 
Approximately $500 \mathrm{~g}$ to $1 \mathrm{~kg}$ of rock were collected for each sample interval. Rock samples were scraped or chiseled in a consistent manner across each interval of uniform lithology in order to obtain a representative single sample of the entire interval. The bulk samples were shipped to the laboratories of the USGS in Denver, Colorado, for sample preparation.

\section{Rock Sample Preparation}

Rock samples were dried in air at ambient temperature. The coarse-fraction samples were disaggregated in a mechanical jaw crusher and a split was then ground in a ceramic plate grinder to $<100$ mesh $(<0.15 \mathrm{~mm})$. Splits of the latter material were provided to various collaborators and to the contract laboratory for analysis. All splits were obtained with a riffle splitter to ensure similarity with the whole sample. A set of splits for all samples was archived. Splits of about $\sim 50 \mathrm{~g}$ were sent to the contract laboratory where they were prepared for analysis.

\section{Analysis}

Samples were analyzed for 40 major, minor, and trace elements using acid digestion in conjunction with inductively coupled plasma-atomic emission spectrometry (ICP-AES). For 40-element analysis (referred to as ICP-40), a split was dissolved using a lowtemperature $\left(<150^{\circ} \mathrm{C}\right)$ digestion with concentrated hydrochloric, hydrofluoric, nitric, and perchloric acids (Crock and others, 1983). The analytical contractor has modified this procedure to shorten the digestion time (P. Lamothe, USGS, oral communication). The acidic sample solution was taken to dryness and the residue was dissolved with $1 \mathrm{ml}$ of aqua regia and then diluted to $10.0 \mathrm{~g}$ with $1 \%$ (volume/volume) nitric acid. This technique also provides analysis of $\mathrm{Bi}$ and $\mathrm{Sn}$. However, an inconsistent bias in the $\mathrm{Bi}$ and $\mathrm{Sn}$ data exists presently for the analytical contractor (P. Lamothe, USGS, oral communication). Consequently, the concentration data for these two elements have been eliminated from the original analytical data set. Sr concentrations are determined in both the ICP-40 and ICP16 (see below) techniques. However, some detection errors occurred in the data set analyzed for Sr by ICP-40 for Section D. Consequently, that set was eliminated from the data files. The ICP-40 technique measures Au above 8 ppm, and Ta above 40 ppm; however, no samples from either of the two sections had concentrations above these detection limits. Consequently, those data were eliminated from the data files.

Another split of the sample was fused in lithium metaborate then analyzed by ICPAES after acid dissolution of the fusion mixture. This technique (referred to as ICP-16) provides a separate analysis of $\mathrm{Si}$, all other major rock-forming elements, and a few trace elements. Most importantly, this is the only analytical technique of those used that measures $\mathrm{Si}$ in these siliceous, phosphatic shale samples. Although the Meade Peak Phosphatic Shale Member is known mostly for its phosphatic content, it also contains minor to important amounts of siliceous components, which result from aluminosilicate minerals, quartz, or biogenic silica. Si measurement is not possible using the 4-acid digestion ICP-40 technique because the $\mathrm{Si}$ is lost as a volatile fluoride compound during digestion. Analysis of major elements using the fusion technique also provides a compositional check on the concentrations of these same elements as measured by acid digestion. $\mathrm{Ti}$ and $\mathrm{Cr}$ were analyzed using both ICP techniques. However, the fusion technique is superior to acid digestion for analysis of resistant minerals containing those elements. Consequently, the analytical data for these two elements using the ICP-40 technique have been eliminated from the data set and only those concentration data for the fusion technique are reported. The ICP-16 technique also measures $\mathrm{Nb}$ above $8 \mathrm{ppm}$; however, no samples from Section D had concentrations above these detection limits. Consequently, those data were eliminated from the data file. 
Se analysis was performed using hydride generation followed by atomic absorption (AA) spectroscopy. The hydride and AA technique also is used for the analysis of As, $\mathrm{Sb}$, and Tl. For the analysis of Se and As, the hydride analytical technique is superior to other analytical techniques. Consequently, the analytical data for Se by energy-dispersive X-ray fluorescence and for As using acid digestion ICP-AES have been eliminated from the data set and only those for the hydride technique are reported.

$\mathrm{Te}$ is measured using AA graphite furnace spectroscopy. Total $\mathrm{S}$ and total $\mathrm{C}$ are measured using combustion in a LECO furnace followed by gas chromatographic measurement. For the other forms of carbon, carbonate carbon is measured as evolved $\mathrm{CO}_{2}$ after acidification of the sample, and organic carbon is calculated as the difference between total and carbonate carbon. Crock and Lichte (1982) and Jackson and others (1988) discuss additional analytical methodology.

$\mathrm{X}$-ray diffraction (XRD) was used to provide a semi-quantitative estimate of apatite and other mineral abundance. In the case of phosphate, this estimate is obtained from the relative peak heights on the $\mathrm{X}$-ray diffractogram of the 21.1 lattice-plane diffraction peak of the apatite. This technique measures only the phosphate associated with the mineral carbonate-fluorapatite, which is the common sedimentary form of apatite in these rocks. In theory, the relative peak height is directly proportional to the concentration of the carbonatefluorapatite. This technique provides a minimum estimate of total phosphate because it is possible that small amounts of phosphate occur in other forms that are not detected by this method. For example, phosphate in organic compounds, amorphous forms, or in minerals other than carbonate-fluorapatite would be not be detected using this $\mathrm{x}$-ray analysis.

Each of the two sections is accompanied by a profile of the equivalent uranium (eU) measurements taken with a gamma-ray spectrometer. Concentrations of eU are given in parts per million (ppm). Section wpsC was measured with an Exploranium GR-320 and section wpsD was measured with a GAD-6 spectrometer. These instruments measure gross gamma-ray flux (including cosmic rays) and provide a quantitative measure of $\mathrm{K}, \mathrm{U}$, and Th. Determination of the abundance of $U$ and $T h$ occurs via detection and counting of gamma rays of specific energy associated with a particular daughter radionuclide for each element, ${ }^{214} \mathrm{Bi}$ with a $1.76 \mathrm{MeV}$ (million electron volt) gamma-ray in the case of uranium. Calculation of total abundance of $U$ and $T h$ assumes secular equilibrium between the measured daughter nuclide and the parent isotope and all intermediate daughter nuclides for each individual element. Potassium abundance is determined from the measurement of gamma rays associated with the decay of ${ }^{40} \mathrm{~K}$. The spectrometer integrates detection over a $2 \pi$ geometry of approximately $1 / 2 \mathrm{~m}^{3}$ and has proportionally higher detection sensitivity to those gamma rays that are emitted closer to the detector. The calibration equations for the two spectrometers assume this geometry on a planar surface and are based on analysis of concrete pads of known composition of the three elements. The calibration coefficients, as well as the constants for subtracted background counts, are a function of latitude, altitude, rock density, and moisture. The coefficients become less reliable as location and rock conditions change from those of the calibration.

In Tysdal and others (1999), we plotted eU concentration data after normalization of the highest eU concentration of section wpsA, $373 \mathrm{ppm}$, to $200 \mathrm{ppm}$ and of section wpsB from a high of $468 \mathrm{ppm}$ to a scaled high value of $282 \mathrm{ppm}$. For the eU data graphed in Tysdal and others (1999) and tabulated in Herring and others (1999) the original eU measurement can be extracted from the plotted values by multiplying by scaling factors of 1.87 for section spsA and 1.66 for section wpsB. This scaling was done because published reports from the 1970's and earlier on uranium and eU concentrations in the Meade Peak Phosphatic Shale Member state that few uranium concentrations from this member exceed 200 ppm (see Swanson, 1970, and references therein) and we had little independent check on accuracy of the spectrometer data. However, new analytical data as part of our study question these past published relationships. 
Recently, we re-analyzed a subset of samples using delayed neutron (DN) analysis, which has a precision of better than 3 percent and an accuracy of generally better than 5 percent (McKown and Millard, 1987). The relationship between the two measurement techniques is shown in figure 2 for 70 samples. The DN analysis can be used to assess the uranium concentration data in Herring and others (1999), which were obtained using ICP40 measurements with a lower detection limit of $100 \mathrm{ppm}$. For a common set of 12 samples where ICP-AES measurements for uranium concentrations are greater than the detection limit of $100 \mathrm{ppm}$, this technique shows that ICP-40 measurements average 12 percent greater than those of DN and have a relative standard deviation of 12 percent. Given this relative credibility in the ICP- 40 technique as verified by $D N$ analysis, the frequency of uranium concentrations $>100 \mathrm{ppm}$ among the set of all composited stratigraphic samples of the Meade Peak Phosphatic Shale Member consequently can be estimated. For 182 channel samples of sections wpsA, wpsB, wpsC, and wpsD as measured by ICP-40, 18 percent of the uranium concentrations are $>100 \mathrm{ppm}$, with 16 percent between 100 and 200 $\mathrm{ppm}$ and 2 percent $>200 \mathrm{ppm}$. These channel samples average over intervals from 1 to 15 feet of true stratigraphic thickness. Clearly, each channel sample will have some uranium concentrations that are indeed higher, perhaps considerably so, than the interval average. Consequently, we believe that uranium concentrations in excess of $200 \mathrm{ppm}$ are not as scarce as reported by Swanson (1970 and references therein) and that uranium concentration measurements from the gamma-ray spectrometers are reasonably correct and should be reported as measured rather than scaling them against an assumed upper limit value.

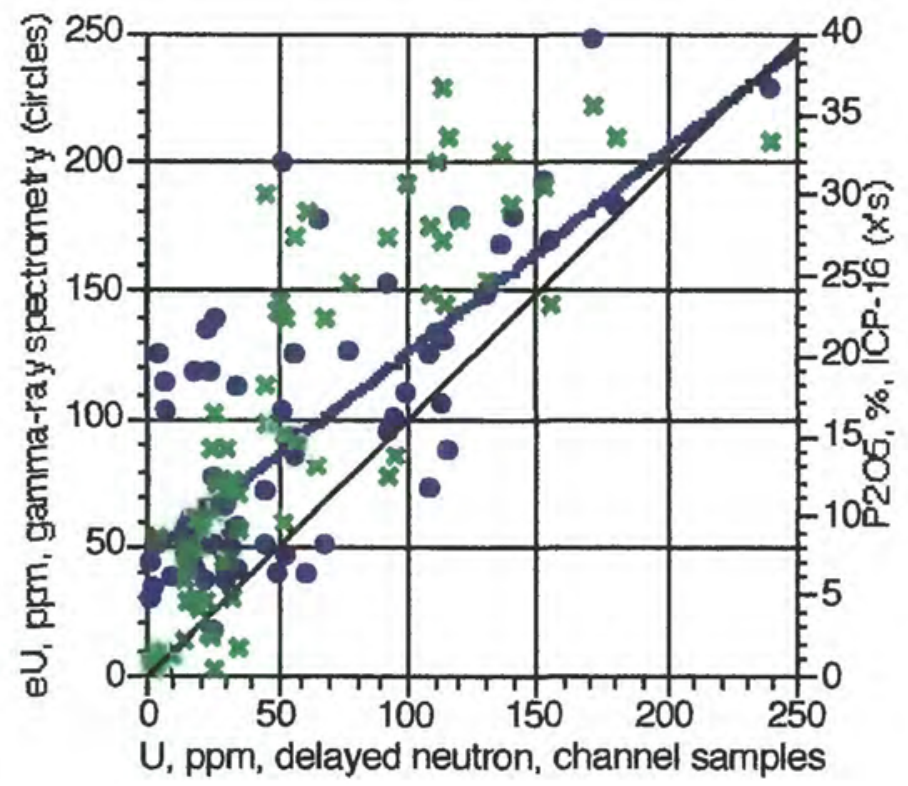

Figure 2. Comparison of measured uranium concentration by delayed neutron analysis in channel samples with gamma-ray spectroscopy measurements taken at 1-foot true-thickness stations through the same intervals and arithmetically averaged (circles). The $1: 1$ and least-squares regression (heavier line; $R^{2}=0.55$ ) lines are shown. Concentrations of $\mathrm{P}_{2} \mathrm{O}_{5}$ in percent are shown for the same samples ( $\mathrm{x}$ 's).

Previous studies of the Phosphoria Formation maintain that there is a consistent relationship between $\mathrm{eU}$ and total uranium contents and between total uranium and phosphate contents (McKelvey, 1956). Our measurements indicate considerable scatter in 
both relationships (fig. 2; Herring and others, 1999; Herring, unpub. data). Measured eU concentrations, even between adjoining 1 foot intervals of consistent lithologic character, often exhibit considerable variability. We expect that this results from: (1) fine-scale variability in the concentration of uranium; (2) the effect of the geometry of the dipping rocks; or (3) from lack of secular equilibrium. Scatter in the $U$ to $\mathrm{P}_{2} \mathrm{O}_{5}$ relationship results from uranium removal or addition by syndepositional effects and (or) by post-depositional alteration, especially weathering. The uranium is mostly located in the phosphate mineral lattice as a substitute for $\mathrm{Ca}$; location of the decay (daughter) products is uncertain. For the phosphatic rocks of the Phosphoria Formation, total gamma-ray counts are dominated by decay of uranium and its various daughter products. $\mathrm{K}_{2} \mathrm{O}$ is generally $<1$ percent in the phosphorite and $<3$ percent in the middle waste shale; Th concentrations are generally $<15$ ppm in ore and waste shale (Altschuler and others, 1958; Swanson, 1970; Herring and others, 1999; Herring, unpub. data).

The measurements for eU were obtained on high-resolution, 1-foot (true-thickness) spacing across both of the sampled sections. These concentration data are graphed in the preliminary report on the stratigraphic descriptions of sections C and D (Tysdal and others, 2000). The eU concentrations reported for the channel-sampled intervals in the data tables are obtained by averaging the 1 -foot measurements over the spacing that corresponds to each channel-sampled interval.

\section{RESULTS}

Analytical results of the rock analyses for the more-weathered stratigraphic section $\mathrm{C}$ and less-weathered section $\mathrm{D}$ (wpsC and wpsD) are listed in data tables 1 and 2 , respectively. The tables include listings of the concentrations of the major rock-forming elements as oxides as well as elements. The oxide concentrations are calculated from the elemental concentrations using standard stoichiometric conversions. Table 1 also includes analytical data for a previously un-analyzed channel sample from section B (wpsB) that extends from the upper waste unit into the overlying Rex Chert Member. Interval base and top footages are specified relative to the stratigraphic base of the Meade Peak Phosphatic Shale Member. This base is defined specifically as the base of the Fish-scale marker stratum, a bioclastic phosphorite unit. Measured footage numbers increase upward through the sections. The concentration data in tables 1 and 2 are listed as reported by the contract laboratory and other collaborators. There has been no statistical manipulation of the data or consideration of qualified values. Qualified values of concentration result from detection of elements that are present but at concentrations less than their lower detection limits (LDL). They are listed in the data table with " $<$ " preceding the LDL. No replacement values for these qualified concentrations, typically done with most traditional data summarization and analysis (for example, see Cohen, 1959), are included.

As a measure of analytical accuracy, a phosphatic rock analytical standard accompanied the rock samples that were submitted to the contract laboratory. The reported analysis and best ongoing average values of this standard is given in table 3 . Analyses of the standards SARM and SARL that are routinely included as a part of the quality control monitoring of the contract laboratory also are included in table 3. Finally, we introduce three carefully prepared standards to be used as ongoing monitors of analytical accuracy for this project (Wilson and others, in preparation). These standards are finely ground splits of composite channel samples of two sections of middle waste rock and one of ore from Section B. This section was described by Tysdal and others (1999) and its analytical data were reported by Herring and others (1999). The preparation and use of these standards are intended to provide better analytical quality control for the project, especially because the standards have similar mineralogy and composition to the typical rocks being analyzed within the project. The first reported set of analyses for these standards is listed in table 3 . 
As a measure of analytical precision, the analytical sample set includes 6 replicated sample pairs for section $C$ and 9 pairs for section $D$. These samples are identified in the data tables. Table 3 includes a listing of the average relative standard difference and average relative standard deviation for each element for all replicated samples that did not have qualified data in their concentrations.

The samples were submitted to the contract laboratory in a randomized sequence. This eliminates systematic errors from sources such as, for example, instrumental drift. The abbreviations for analytical techniques in the column headings of tables 1,2 , and 3 for analytical methodology are defined as follows:

XRD: X-ray diffraction

Hydr. AA: hydride generation followed by atomic absorption

CVAA: cold vapor atomic absorption

ICP-16: inductively-coupled plasma spectrometry, fusion digestion

ICP-40: inductively-coupled plasma spectrometry, acid digestion.

Concentrations of various elements in the channel samples of the two sections are graphed in figure 3. The few "less-than" concentrations reported for some of these elements have been replaced with their lower detection limits for graphing.

\section{ACKNOWLEDGMENTS}

The sections were measured within the Dry Valley mine, operated by the FMC Corporation. We thank FMC Corporation for providing access and we thank company personnel who freely discussed the geology of the area. P. Lamothe provided helpful insights into the quality of the analytical data. We appreciate help in sample preparation by D. Firewick, B. Nigol, N. Nigol, and S. Herring. M. Fallin assisted with data table preparation. 


\section{REFERENCES CITED}

Altschuler, Z.S., Clarke, R.S. and Young, E.J., 1958, Geochemistry of uranium in apatite and phosphorite: U.S. Geological Survey Professional Paper 314D, p. 45-90.

Cohen, A.C., Jr., 1959, Simplified estimators for the normal distribution when samples are singly censored or truncated: Technometrics, vol. 1, p. 217-237.

Cressman, E.R., and Swanson, R.W., 1964, Stratigraphy and petrology of the Permian rocks of southwestern Montana: U.S. Geological Survey Prof essional Paper 313-C, p. 275-569.

Crock, J.G. and Lichte, F.E., 1982, An improved method for the determination of trace levels of arsenic and antimony in geologic materials by automated hydride generationatomic absorption spectroscopy: Analytica Chimica Acta, vol. 144, p. 223-233.

Crock, J.G., Lichte, F.E., and Briggs, P.E., 1983, Determination of elements in National Bureau of Standards Geologic Reference Materials SRM 278 obsidian and SRM 688 basalt by inductively coupled argon plasma-atomic emission spectrometry: Geostandards Newsletter, vol. 7, p. 335-340.

Gulbrandsen, R.A., 1966, Chemical composition of phosphorites of the Phosphoria Formation: Geochimica et Cosmochimica Acta, v. 30, no. 8, p. 769-778.

Gulbrandsen, R.A., 1975, Analytical data on the Phosphoria Formation, western United States: U.S. Geological Survey Open-File Report 75-554, 45 p.

Gulbrandsen, R.A., 1979, Preliminary analytical data on the Meade Peak member of the Phosphoria Formation at Hot Springs underground mine, Trail Canyon trench, and Conda underground mine, southeastern Idaho: U.S. Geological Survey Open-File Report 79-369, 35 p.

Gulbrandsen, R.A., and Krier, D.J., 1980, Large and rich phosphorus resources in the Phosphoria Formation in the Soda Springs area southeastern Idaho: U.S. Geological Survey Bulletin 1496, 25 p.

Hale, L.A., 1967, Phosphate exploration using gamma radiation logs, Dry Valley, Idaho, in Hale, L.A., ed., Anatomy of the western phosphate field: Salt Lake City, Intermountain Association of Field Geologists, 15th Annual Field Conference Guidebook, p. 147-159.

Herring, J.R., Desborough, G.A., Wilson, S.A., Tysdal, R.G., Grauch, R.I., and Gunter, M.E., 1999, Chemical composition of weathered and unweathered strata of the Meade Peak Phosphatic Shale Member of the Permian Phosphoria Formation. A. Measured sections A and B, central part of Rasmussen Ridge, Caribou County, Idaho: U.S. Geological Survey Open-File Report 99-147-A, 24 p.

Jackson, L.L., Brown, F.W., and Neil, S.T., 1988, Major and minor elements requiring individual determinations, classical whole rock analysis, and rapid rock analysis, p. G1-G23, in Baedecker, P.A., (ed.), Geochemical Methods of Analysis: U.S. Geological Survey Bulletin 1770. 
McKelvey, V.E., 1956, Uranium in phosphate rock, in Page, L.R., Stocking, H.E., and Smith, H.B., compilers, Contribution to the geology of uranium and thorium by the United States Geological Survey and Atomic Energy Commission for the United Nations International Conference on Peaceful Uses of Atomic Energy, Geneva, Switzerland, 1955: U.S. Geological Survey Professional Paper 300, p. 477-481.

McKelvey, V.E., Williams, J.S., Sheldon, R.P., Cressman, E.R., Cheney, T.M., and Swanson, R.W., 1959, The Phosphoria, Park City, and Shedhorn Formations in the Western Phosphate Field: U.S. Geological Survey Professional Paper 313-A, 47 p.

McKown, D.M., and Millard, H.T., Jr., 1987, Determination of uranium and thorium by delayed neutron counting, in Baedecker, P.A., ed., Methods for geochemical analysis: U.S. Geological Survey Bulletin B1770, p. I1-I12.

Rioux, R.L., Hite, R.J., Dyni, J.R., and Gere, W.C., 1975, Geologic map of the Upper Valley quadrangle, Caribou County, Idaho: U.S. Geological Survey Geologic Quadrangle Map GQ-1194, scale 1:24,000.

Service, A.L., 1966, An evaluation of the western phosphate industry and its resources, Part 3. Idaho: U.S. Bureau of Mines Report of Investigations 6801, 201 p.

Swanson, R.G., 1970, Mineral resources in Permian rocks of southwest Montana: U.S. Geological Survey Professional Paper 313-E, p. 661-777.

Tysdal, R.R, Johnson, E.A., Herring, J.R., and Desborough, G.A., 1999, Stratigraphic sections and equivalent uranium (eU), Meade Peak Phosphatic Shale Member of Permian Phosphoria Formation, central part of Rasmussen Ridge, Caribou County, Idaho: U.S. Geological Survey Open-file Report 99-20, 1 plate.

Tysdal, R.G., Desborough, G.A., Herring, J.R., Grauch, R.I., and Stillings, L.A., 2000, Stratigraphic sections and equivalent uranium (eU), Meade Peak Phosphatic Shale Member of Permian Phosphoria Formation, Dry Valley, Caribou County, Idaho: U.S. Geological Survey Open-file Report 99-20-B, 1 plate.

Wilson, S.A., Herring, J.R. and others, in preparation, New phosphatic rock standards from middle waste rock and ore sections of the Meade Peak Member of the Phosphoria Formation. 
Section C
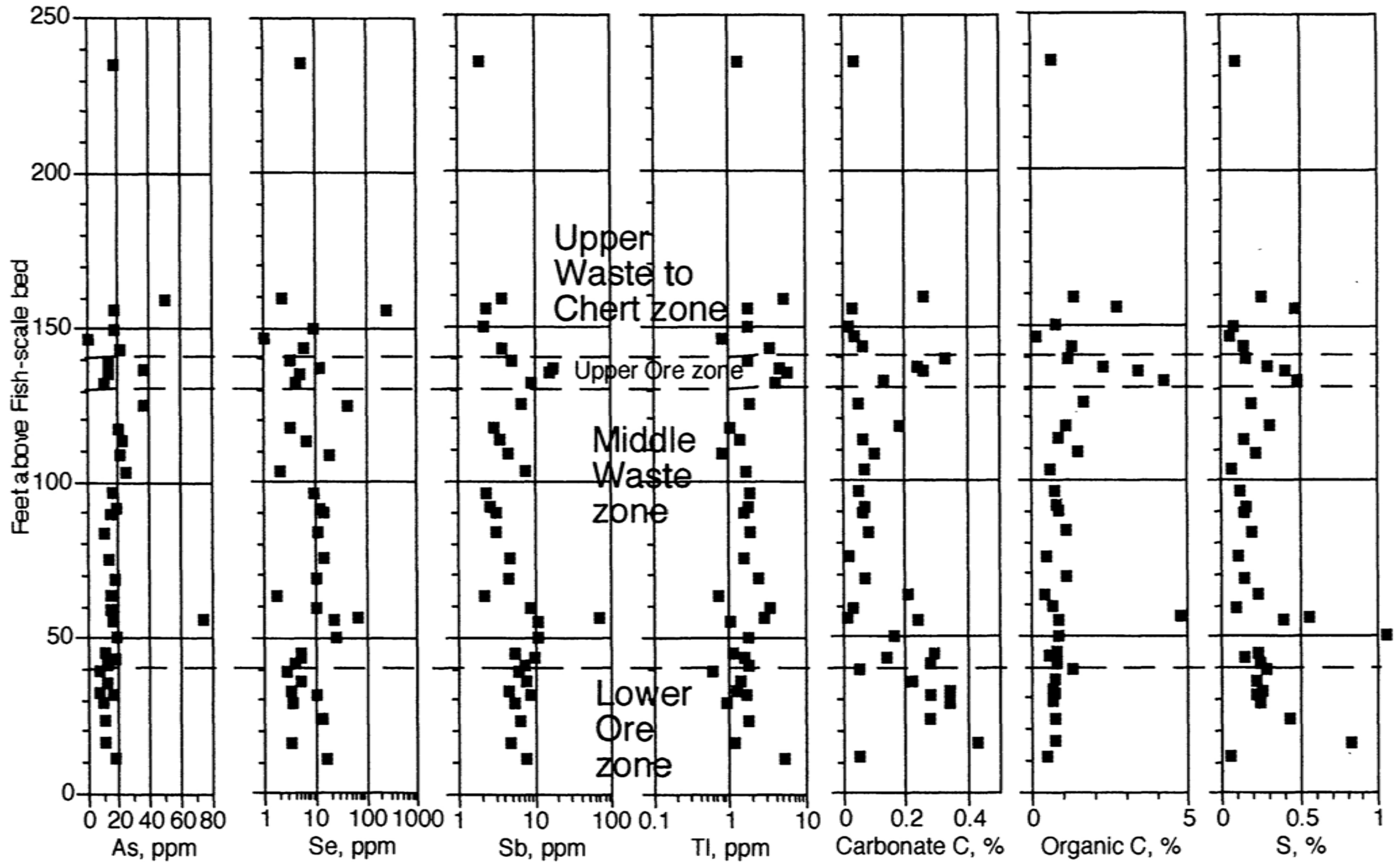
Section C

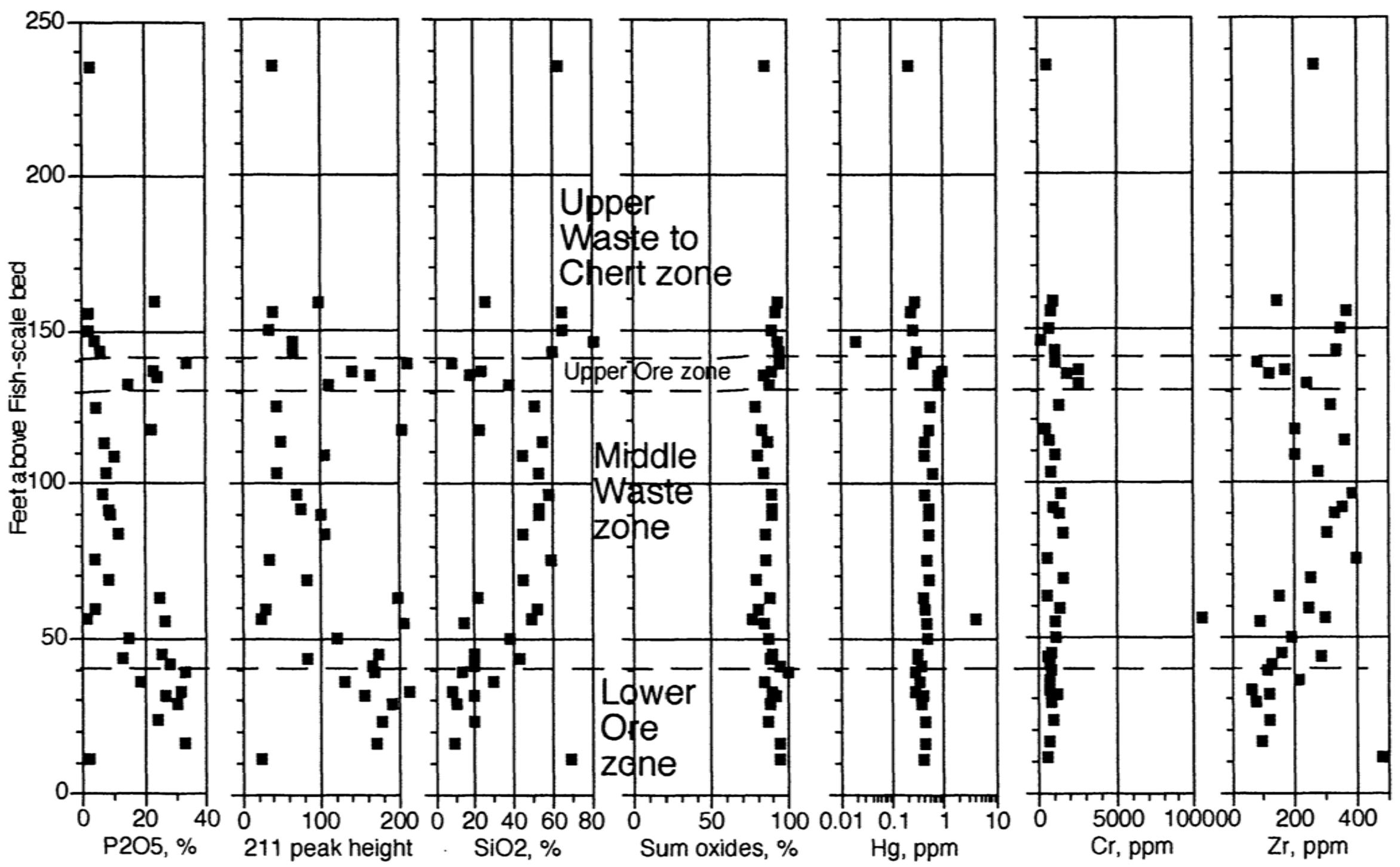


Section C

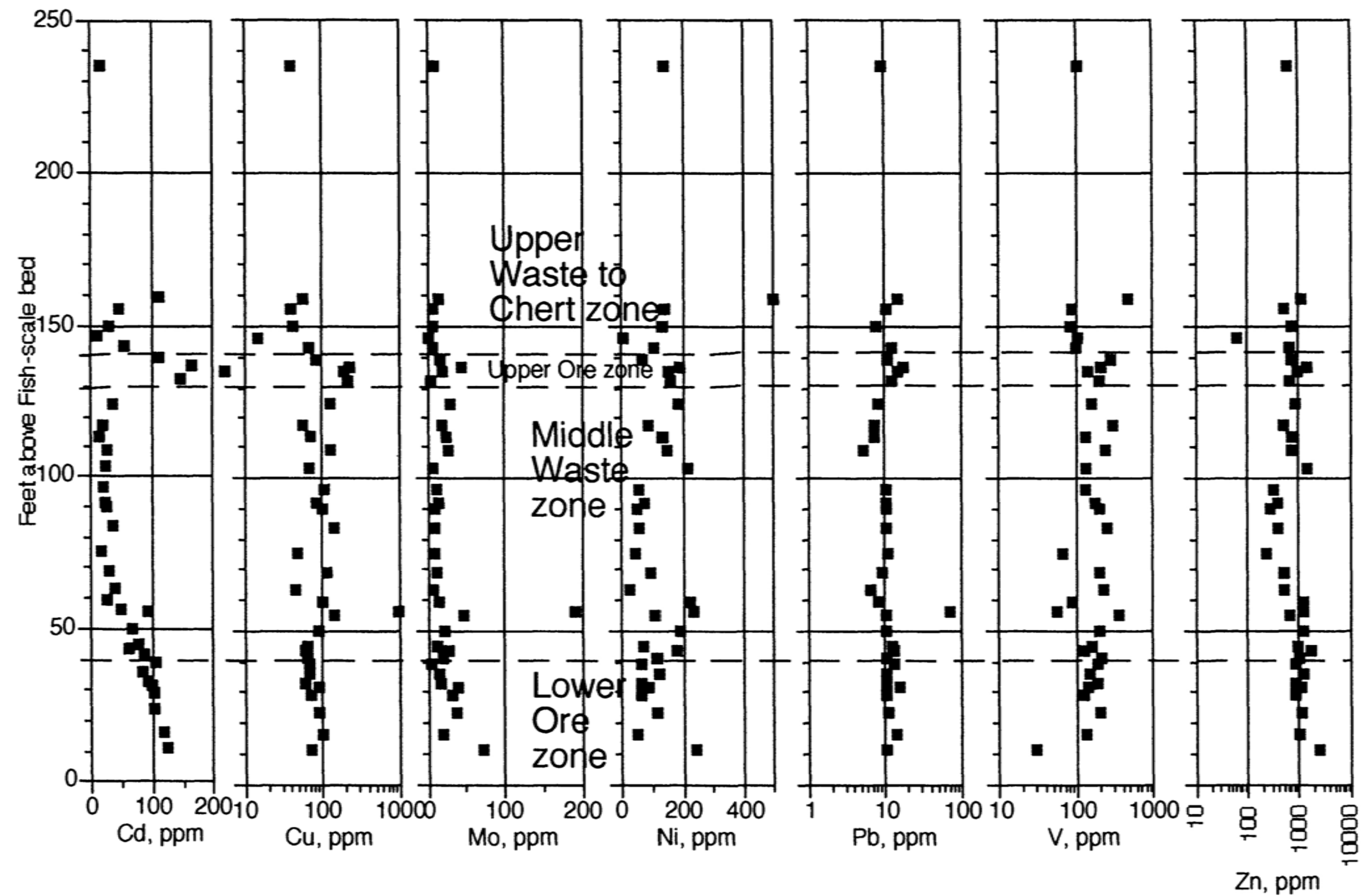


Section D
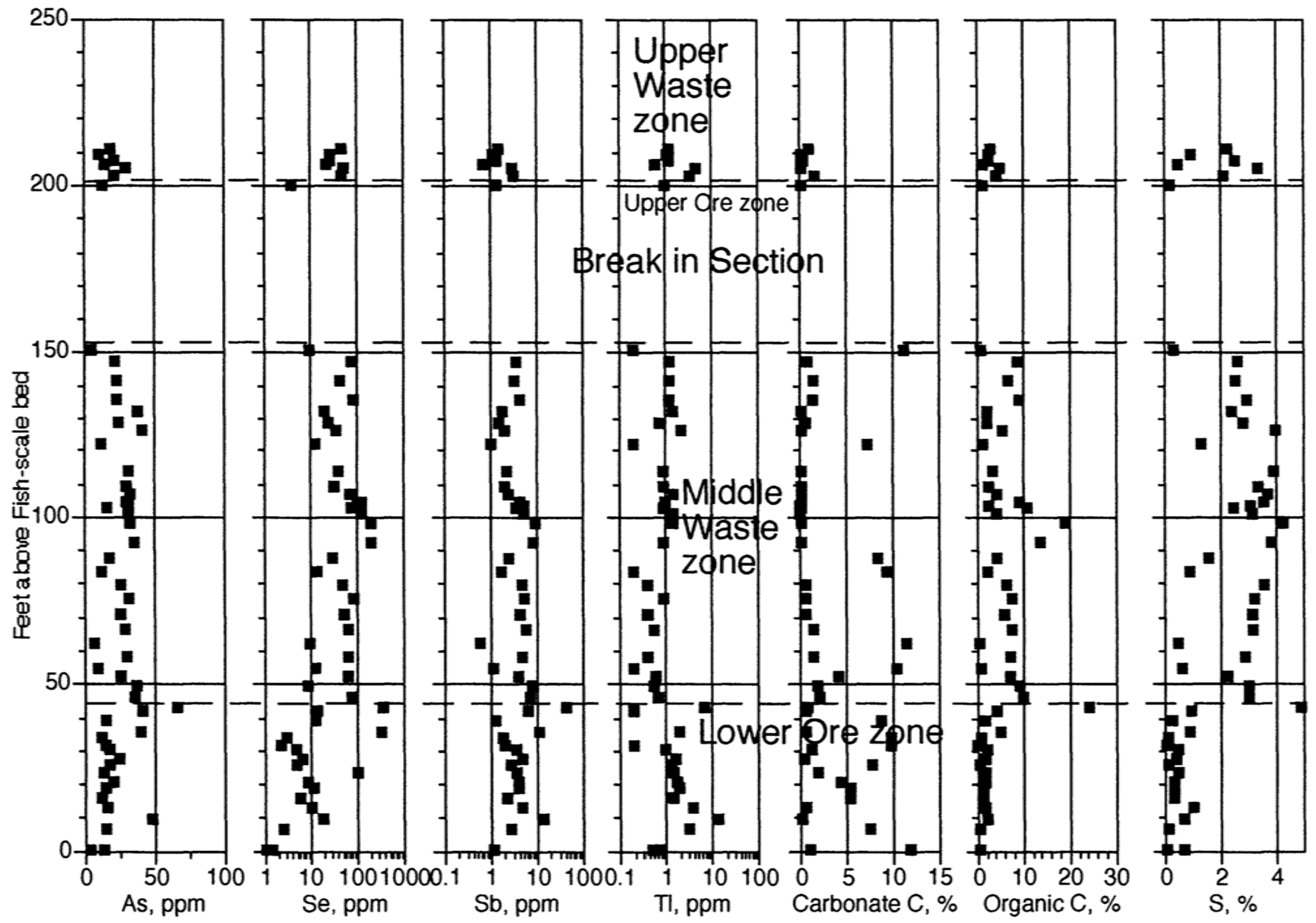

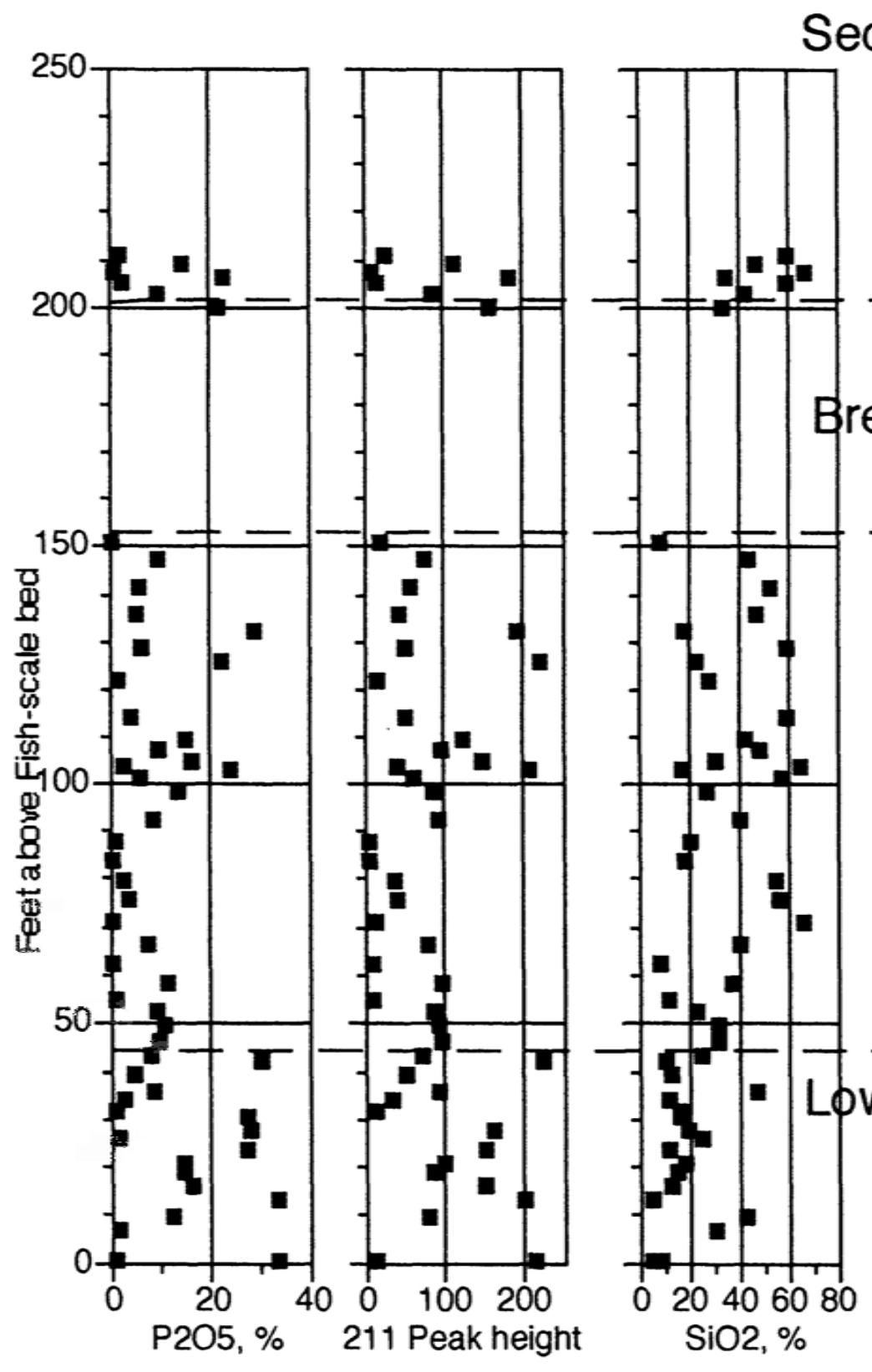

Section D
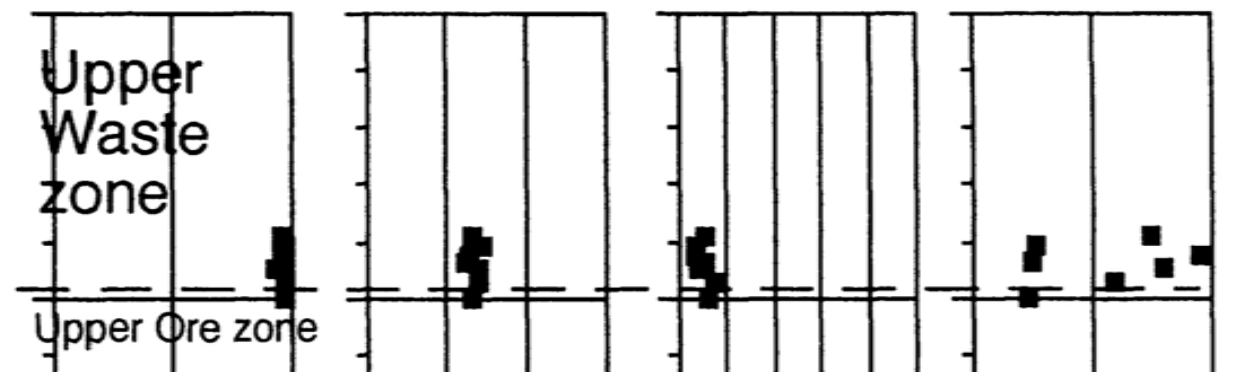

Break in Section
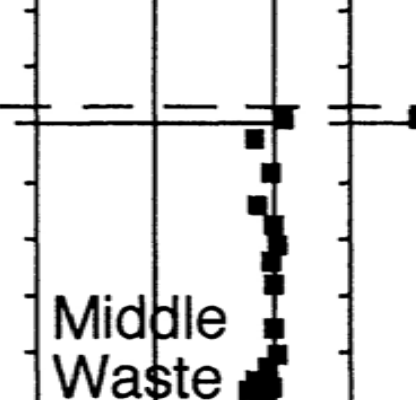


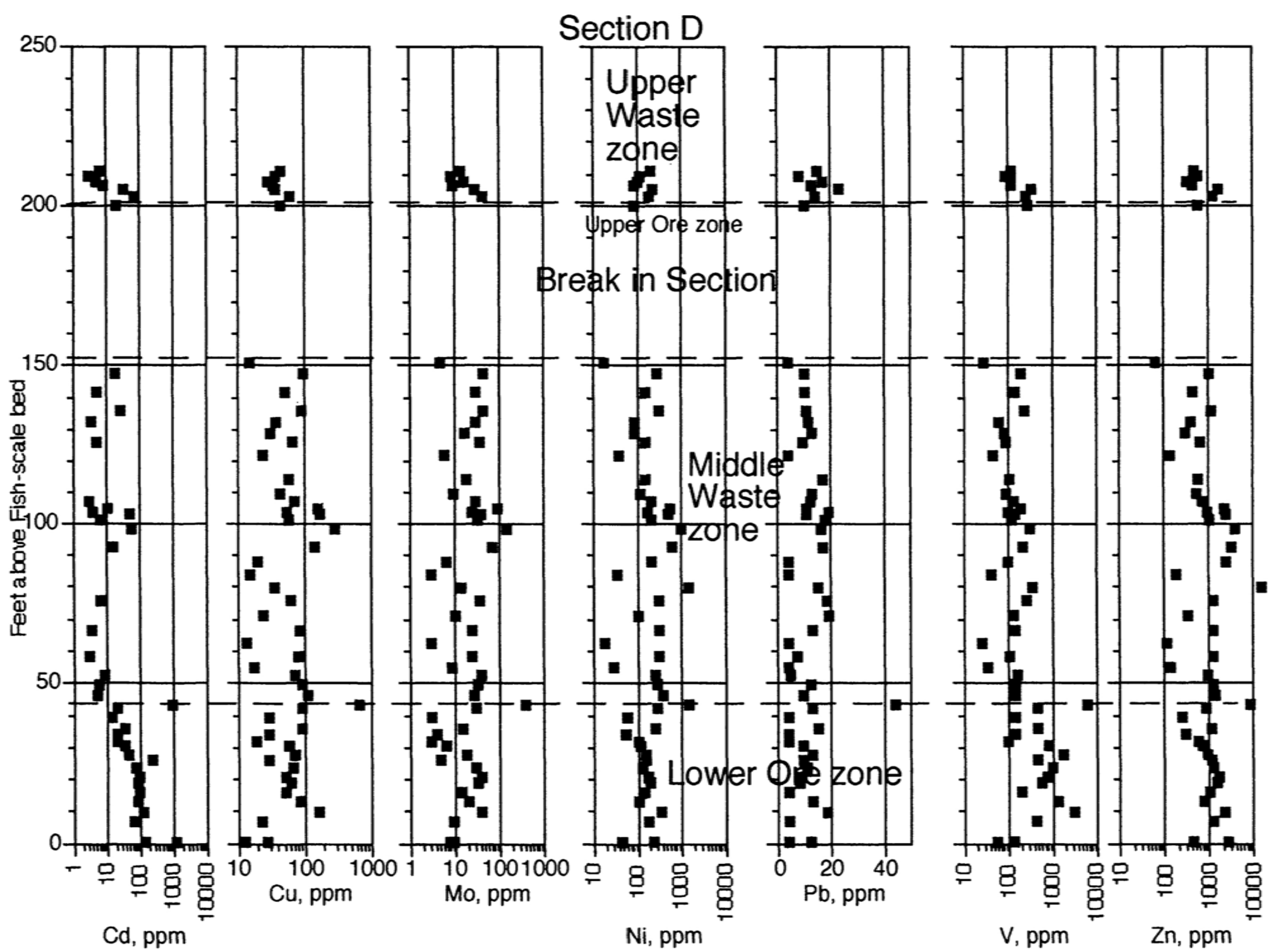




\begin{tabular}{|c|c|c|c|c|c|c|c|c|c|c|c|c|c|}
\hline $\begin{array}{l}\text { Section C } \\
\text { Field No. }\end{array}$ & Lab No. & $\begin{array}{c}\text { Unit within Meade Peak } \\
\text { Member }\end{array}$ & Lithology & $\begin{array}{l}\text { Interval } \\
\text { base, ft }\end{array}$ & $\begin{array}{c}\text { Interval } \\
\text { top, } \mathrm{ft}\end{array}$ & $\begin{array}{c}\text { Interval } \\
\text { thickness, ft }\end{array}$ & $\begin{array}{l}\text { Interval } \\
\text { midpoint, } \mathrm{ft}\end{array}$ & $\begin{array}{l}\text { Se, ppm, } \\
\text { Hyd. }\end{array}$ & $\begin{array}{l}\text { As, ppm, } \\
\text { hydride }\end{array}$ & $\begin{array}{l}\mathrm{Hg}, \mathrm{ppm}, \\
\text { CVAA }\end{array}$ & $\begin{array}{l}\text { Sb, ppm, } \\
\text { Hyd. }\end{array}$ & $\begin{array}{l}\text { Te, ppm, } \\
\text { FAA }\end{array}$ & $\begin{array}{l}\text { TI, ppm, } \\
\text { ICP-MS }\end{array}$ \\
\hline WPSCO11C & $C-123865$ & Footwall Mudstone & mudstone & 10 & 12 & 2 & 11.0 & 17.2 & 17.9 & 0.4 & 7.8 & 0.3 & 5.2 \\
\hline WPSCO15C & $C-123849$ & Lower Ore zone, A-Bed Ore & phosphorite & 12 & 20 & 8 & 16.0 & 3.2 & 11.1 & 0.44 & 4.5 & $<0.1$ & 0.9 \\
\hline WPSCO15X & $C-123871$ & & \multicolumn{3}{|c|}{ duplicate of previous sample } & & 16.0 & 3.2 & 11.4 & 0.43 & 5.1 & $<0.1$ & 1.4 \\
\hline WPSCO2OC & $C-123877$ & Lower Ore zone, A-Cap & mudstone & 20 & 26.3 & 6.3 & 23.2 & 14.3 & 11.8 & 0.42 & 6.4 & $<0.1$ & 1.7 \\
\hline WPSCO28C & $C-123856$ & Lower Ore zone, Lower B Ore & phosphorite & 26.3 & 31 & 4.7 & 28.7 & 3.5 & 10.6 & 0.34 & 5.5 & 0.2 & 0.9 \\
\hline WPSCO31C & $C-123876$ & Lower Ore zone, B-Mudstone & phosphorite & 31 & 31.5 & 0.5 & 31.3 & 10.2 & 16 & 0.39 & 8.6 & 0.3 & 1.6 \\
\hline WPSC032C & $C-123867$ & Lower Ore zone, Upper B Ore & phosphorite & 31.5 & 33 & 1.5 & 32.3 & 3.3 & 7.1 & 0.27 & 4.5 & 0.3 & 1.2 \\
\hline WPSCO35C & $C-123857$ & Lower Ore zone, False Cap & mudstone & 33 & 38 & 5 & 35.5 & 5.3 & 12.6 & 0.32 & 7.9 & 0.1 & 1.4 \\
\hline WPSCO39C & $C-123866$ & Lower Ore zone, C-Bed & phosphorite & 38 & 40 & 2 & 39.0 & 2.9 & 8.1 & 0.28 & 6 & $<0.1$ & 0.6 \\
\hline WPSCO41C & $C-123873$ & Middle Waste & mudstone & 40 & 42 & 2 & 41.0 & 3.8 & 12.5 & 0.35 & 7.3 & 0.3 & 1.7 \\
\hline WPSCO $43 \mathrm{C}$ & C- 123842 & Middle Waste & mudstone & 42 & 44 & 2 & 43.0 & 5.4 & 17.3 & 0.29 & 9.6 & 0.2 & 1.5 \\
\hline WPSCO45C & $C-123852$ & Middie Waste & phosphorite & 44 & 45 & 1 & 44.5 & 5.4 & 11.8 & 0.29 & 5.5 & $<0.1$ & 1.1 \\
\hline WPSCOSOC & $C-123875$ & Middle Waste & \begin{tabular}{|l|l} 
mudstone & \\
\end{tabular} & 45 & 54.5 & 9.5 . & 49.8 & 24.7 & 20.3 & 0.46 & 11.5 & 0.2 & 1.6 \\
\hline WPSCOSOX & $C-123885$ & & \multicolumn{3}{|c|}{ duplicate of previous sample } & & 49.8 & 27 & 17.9 & 0.46 & 10.7 & 0.2 & 1.8 \\
\hline WPSCOSSC & $\mathrm{C}-123882$ & Middle Waste & phosphorite & 54.5 & 55.5 & 1 & 55.0 & 24.4 & 16.1 & 0.46 & 11.2 & $<0.1$ & 1 \\
\hline WPSC056C & $C-123881$ & Middle Waste & carbon seam & 55.5 & 55.8 & 0.3 & 55.7 & 70.3 & 74.1 & 4.22 & 71 & 0.2 & 2.8 \\
\hline WPSCO6OC & $C-123884$ & Middle Waste & mudstone & 55.8 & 62 & 6.2 & 58.9 & 10.5 & 15.6 & 0.41 & 8.8 & 0.3 & 3.4 \\
\hline WPSCO63C & $C-123861$ & Middle Waste & \begin{tabular}{|l|l} 
chert & \\
\end{tabular} & 62 & 64 & 2 & 63.0 & 1.9 & 16.5 & 0.39 & 2.3 & $<0.1$ & 0.7 \\
\hline WPSC063X & C-123864 & & \multicolumn{3}{|c|}{ duplicate of previous sample } & & 63.0 & 1.8 & 14.7 & 0.41 & 2.1 & 0.3 & 0.7 \\
\hline WPSCO7OC & $C-123854$ & Middle Waste & \begin{tabular}{|l|} 
mudstone \\
\end{tabular} & 64 & 73 & 9 & 68.5 & 10.7 & 17.6 & 0.52 & 4.6 & 0.1 & 2.4 \\
\hline WPSCO75C & $\mathrm{C}-123843$ & Middle Waste & \begin{tabular}{|l|l} 
dolomite \\
\end{tabular} & 73 & 77 & 4 & 75.0 & 16.1 & 12.8 & 0.45 & 4 & $<0.1$ & 1.4 \\
\hline WPSC075X & $C-123855$ & & \multicolumn{3}{|c|}{ duplicate of previous sample } & & 75.0 & 14.8 & 15 & 0.43 & 5.4 & 0.1 & 1.6 \\
\hline WPSCO80C & $C-123863$ & Middle Waste & mudstone & 77 & 89 & 12 & 83.0 & 12.1 & 11.5 & 0.52 & 3.1 & 0.6 & 1.8 \\
\hline WPSCO9OC & $C-123850$ & Middle Waste & nodular phosp & 89 & 90 & 1 & 89.5 & 15.2 & 15.4 & 0.49 & 3.2 & $<0.1$ & 1.5 \\
\hline WPSC091C & $C-123845$ & Middle Waste & mudstone & 90 & 92.25 & 2.25 & 91.1 & 12.3 & 19.4 & 0.5 & 2.7 & 0.1 & 1.7 \\
\hline WPSCO96C & $C-123880$ & Middle Waste & mudstone, dol & 92.25 & 100 & 7.75 & 96.1 & 9.5 & 16.4 & 0.42 & 2.4 & $<0.1$ & 1.8 \\
\hline WPSC104C & $C-123879$ & Middle Waste & \begin{tabular}{|l|l} 
mudstone \\
\end{tabular} & 100 & 106 & 6 & 103.0 & 2.1 & 24.6 & 0.62 & 7.7 & $<0.1$ & 1.6 \\
\hline WPSC108C & $C-123859$ & Middle Waste & mudstone & 106 & 110.5 & 4.5 & 108.3 & 19.7 & 21.6 & 0.41 & 4.5 & $<0.1$ & 0.8 \\
\hline WPSC112C & C-123878 & Middle Waste & mudstone, pho & 110.5 & 115.2 & 4.7 & 112.9 & 6.7 & 23.2 & 0.43 & 3.5 & $<0.1$ & 1.4 \\
\hline WPSC116C & $C-123860$ & Middle Waste & Phosphorite & 115.25 & 118.5 & 3.25 & 116.9 & 3.3 & 20.7 & 0.49 & 3 & $<0.1$ & 1 \\
\hline WPSC122C & $C-123858$ & Middle Waste & mudstone, pho & 118.5 & 130 & 11.5 & 124.3 & 43.2 & 36 & 0.55 & 6.8 & $<0.1$ & 1.9 \\
\hline WPSC133C & $C-123851$ & Upper Ore zone, D-1 Ore & phosphorite & 130 & 134 & 4 & 132.0 & 4.5 & 11.1 & 0.76 & 9 & 0.2 & 3.7 \\
\hline WPSC133X & C- 123848 & & \multicolumn{3}{|c|}{ duplicate of previous sample } & & 132.0 & 4.3 & 11.6 & 0.75 & 9.6 & $<0.1$ & 4.3 \\
\hline WPSC135C & $C-123853$ & Upper Ore zone, D-2 Ore & phosphorite & 134 & 136.3 & 2.3 & 135.2 & 5.1 & 13.8 & 0.78 & 15.8 & 0.2 & 5.8 \\
\hline WPSC137C & $C-123870$ & Upper Ore zone, D-Mudstone & mudstone & 136.3 & 137 & 0.7 & 136.7 & 12.7 & 36.6 & 0.91 & 17.8 & 0.4 & 4.4 \\
\hline WPSC138C & $C-123868$ & Upper Ore zone, D-3 Ore & phosphorite & 137 & 141 & 4 & 139.0 & 3.3 & 14.4 & 0.25 & 5.2 & 0.2 & 1.7 \\
\hline WPSC1 $144 C$ & $C-123847$ & Upper Waste & mudstone & 141 & 145 & 4 & 143.0 & 6.3 & 21.8 & 0.31 & 3.9 & 0.1 & 3.3 \\
\hline WPSC146C & C-123862 & Upper Waste & \begin{tabular}{|l|l} 
chert \\
\end{tabular} & 145 & 147 & 2 & 146.0 & 1 & 1.3 & 0.02 & $<0.6$ & $<0.1$ & 0.8 \\
\hline WPSC15OC & $C-123869$ & Upper Waste & mudstone, phe & 147 & 152 & 5 & 149.5 & 9.6 & 17.8 & 0.26 & 2.2 & 0.3 & 1.9 \\
\hline WPSC150X & $C-123886$ & & \multicolumn{3}{|c|}{ duplicate of previous sample } & & 149.5 & 10.2 & 17 & 0.25 & 2.2 & 0.2 & 1.6 \\
\hline WPSC15SC & $C-123846$ & Upper Waste & mudstone, silt & 152 & 158.5 & 6.5 & 155.3 & 259 & 17.4 & 0.22 & 2.3 & 0.1 & 1.7 \\
\hline WPSC159C & $C-123872$ & F-Bed & phosphorite & 158.5 & 159 & 0.5 & 158.8 & 2.3 & 50 & 0.27 & 3.9 & $<0.1$ & 5.1 \\
\hline WPSC17OC & C-123844 & Upper Waste to chert & & 159 & 310 & 151 & 234.5 & 5.6 & 17.3 & 0.21 & 2 & 0.2 & 1.3 \\
\hline Section B & & & & & & & & & & & & & \\
\hline WPSB $170 C$ & $C-123883$ & Upper Waste to chert & mudstone & 163 & 190 & 27 & 176.5 & 47.9 & 9.7 & 0.2 & 1.7 & 0.5 & 2.1 \\
\hline
\end{tabular}

Table 1, Page 20 


\begin{tabular}{|c|c|c|c|c|c|c|c|c|c|c|c|c|c|c|c|c|}
\hline $\begin{array}{l}\text { Section C } \\
\text { Field No. }\end{array}$ & $\begin{array}{c}\text { C, \%, } \\
\text { Combustion }\end{array}$ & $\begin{array}{c}\mathrm{CO} 2, \%, \\
\text { Acidification }\end{array}$ & $\begin{array}{c}\text { Carbonate C, } \\
\%, \\
\text { Acidification }\end{array}$ & $\begin{array}{c}\text { Organic } \mathrm{C}, \%, \\
\text { difference }\end{array}$ & $\begin{array}{c}\mathrm{S}, \%, \\
\text { Combustion }\end{array}$ & $\begin{array}{c}\text { Al, \%, ICP- } \\
16\end{array}$ & $\begin{array}{c}\mathrm{Ca}, \%, 10 \mathrm{P} \\
16\end{array}$ & Fe, $\%$, ICP. & $\begin{array}{c}\mathrm{K}, \%, 1 C \mathbf{}- \\
16\end{array}$ & $\begin{array}{c}\mathrm{Mg}, \%, I C P \\
16\end{array}$ & $\begin{array}{c}\mathrm{Na}, \%, 1 C \mathrm{P} \\
16\end{array}$ & $\begin{array}{c}P, \%, 1 C P- \\
16\end{array}$ & $\begin{array}{c}\mathrm{Si}, \%, 1 \mathrm{CP}- \\
16\end{array}$ & $\begin{array}{c}\text { Ti, \%, ICP- } \\
16\end{array}$ & $\begin{array}{c}\text { AlOx, \%, } \\
1 C P-16\end{array}$ & $\begin{array}{c}\mathrm{CaOx}, \%, \\
\mathrm{ICP}-16\end{array}$ \\
\hline WPSCO11C & 0.5 & \begin{tabular}{|l|}
0.17 \\
\end{tabular} & \begin{tabular}{|l|}
0.05 \\
\end{tabular} & 0.45 & 0.05 & 5.85 & 2.43 & 2.48 & \begin{tabular}{|l|}
2.15 \\
\end{tabular} & 0.46 & \begin{tabular}{l|} 
\\
\end{tabular} & 0.96 & 32.3 & 0.46 & 11.1 & 3.4 \\
\hline WPSCO15C & 1.13 & 1.62 & 0.44 & 0.69 & 0.83 & 1.09 & 33.2 & 0.55 & 0.32 & 0.16 & 0.68 & 14.2 & 4.64 & 0.06 & 2.1 & 46.4 \\
\hline WPSC015X & 1.15 & 1.53 & 0.42 & 0.73 & 0.81 & 1.05 & 33.3 & 0.55 & 0.31 & 0.16 & 0.65 & 14.6 & 4.43 & 0.06 & 2.0 & 46.6 \\
\hline WPSCO2OC & 1.01 & 1.02 & 0.28 & 0.73 & 0.44 & 2.01 & 24.5 & 0.92 & 0.78 & 0.21 & 0.32 & 10.6 & 9.42 & 0.12 & 3.8 & 34.3 \\
\hline WPSCO28C & 1 & 1.26 & 0.34 & 0.66 & 0.24 & 1.13 & 30.4 & 0.57 & 0.4 & 0.14 & 0.19 & 13.4 & 5.1 & 0.06 & 2.1 & 42.5 \\
\hline WPSCO31C & 1.02 & 1.04 & 0.28 & 0.74 & 0.22 & 1.93 & 26.9 & 0.97 & 0.91 & 0.22 & 0.19 & 11.8 & 9.42 & 0.13 & 3.6 & 37.6 \\
\hline WPSCO32C & 1.02 & 1.26 & 0.34 & 0.68 & 0.26 & 0.93 & 31.9 & 0.51 & 0.33 & 0.11 & 0.19 & 13.9 & 4.02 & 0.05 & 1.8 & 44.6 \\
\hline WPSCO35C & 0.96 & 0.79 & 0.22 & 0.74 & 0.22 & 2.69 & 18.5 & 1.27 & 1.2 & 0.21 & 0.2 & 8.08 & 14.3 & 0.22 & 5.1 & 25.9 \\
\hline WPSCO39C & 1.34 & 0.17 & 0.05 & 1.29 & 0.28 & 1.26 & 33 & 0.71 & 0.57 & 0.14 & 0.21 & 14.5 & 6.61 & 0.09 & 2.4 & 46.2 \\
\hline WPSCO41C & 1.04 & 1.01 & 0.28 & 0.76 & 0.24 & 1.93 & 27.7 & 1 & 0.84 & 0.16 & 0.2 & 12.3 & 9.24 & 0.13 & 3.6 & 38.8 \\
\hline WPSCO43C & 0.69 & 0.51 & 0.14 & 0.55 & 0.14 & 3.82 & 13.1 & 1.78 & 1.68 & 0.22 & 0.16 & 5.62 & 20.4 & 0.32 & 7.2 & 18.3 \\
\hline WPSCO45C & 1.08 & 1.07 & 0.29 & 0.79 & 0.23 & 1.84 & 25.7 & 0.91 & 0.8 & 0.17 & 0.2 & 11.2 & 9.49 & 0.14 & 3.5 & 36.0 \\
\hline WPSCOSOC & 1.05 & 0.6 & 0.16 & 0.89 & 1.89 & 4.07 & 15 & 1.75 & 1.97 & 0.22 & 0.22 & 6.51 & 18.5 & 0.26 & 7.7 & 21.0 \\
\hline WPSCOSOX & 1.03 & 0.63 & 0.17 & 0.86 & 0.21 & 3.73 & 14.2 & 1.65 & 1.86 & 0.21 & 0.22 & 6.32 & 17.4 & 0.24 & 7.0 & 19.9 \\
\hline WPSCOS5C & 1.08 & 0.89 & 0.24 & 0.84 & 0.4 & 1.61 & 26.2 & 1.13 & 0.73 & 0.12 & 0.26 & 11.7 & 6.86 & 0.09 & 3.0 & 36.7 \\
\hline WPSCO56C & 4.74 & 0.05 & 0.01 & 4.73 & 0.56 & 5.79 & 2.46 & 3.35 & 2.52 & 0.85 & 0.12 & 0.71 & 23.3 & 0.38 & 10.9 & 3.4 \\
\hline WPSCO60C & 0.69 & 0.12 & 0.03 & 0.66 & 0.09 & 6.22 & 3.3 & 2.42 & 1.88 & 0.21 & 0.24 & 1.65 & 24.6 & 0.36 & 11.7 & 4.6 \\
\hline WPSCO63C & 0.63 & 0.78 & 0.21 & 0.42 & 0.23 & 2.1 & 20.9 & 2.13 & 0.76 & 0.09 & 0.21 & 9.87 & 10.1 & 0.16 & 4.0 & 29.2 \\
\hline WPSC063X & 0.64 & 0.75 & 0.2 & 0.44 & 0.23 & 2.31 & 25 & 2.32 & 0.84 & 0.11 & 0.24 & 11.8 & 10.7 & 0.17 & 4.4 & 35.0 \\
\hline WPSCO7OC & 1.14 & 0.24 & 0.07 & 1.07 & 0.15 & 4.45 & 7.35 & 2.16 & 1.5 & 0.2 & 0.31 & 3.59 & 21.3 & 0.31 & 8.4 & 10.3 \\
\hline WPSCO75C & 0.49 & 0.07 & 0.02 & 0.47 & 0.12 & 5.15 & 3.05 & 2.19 & 2.15 & 0.16 & 0.27 & 1.78 & 26.7 & 0.44 & 9.7 & 4.3 \\
\hline WPSCO75X & 0.47 & 0.08 & 0.02 & 0.45 & 0.08 & 5.34 & 3.27 & 2.51 & 2.3 & 0.17 & 0.25 & 1.94 & 28.5 & 0.46 & 10.1 & 4.6 \\
\hline WPSCO $80 \mathrm{C}$ & 1.21 & 0.29 & 0.08 & 1.13 & 0.19 & 4.07 & 10.8 & 2.11 & 1.47 & 0.25 & 0.21 & 4.99 & 21.2 & 0.31 & 7.7 & 15.1 \\
\hline WPSCO9OC & 0.88 & 0.21 & 0.06 & 0.82 & 0.14 & 4.55 & 8.26 & 2.11 & 1.78 & 0.19 & 0.3 & 3.86 & 25.1 & 0.38 & 8.6 & 11.6 \\
\hline WPSCO91C & 0.88 & 0.24 & 0.07 & 0.81 & 0.16 & 4.43 & 8.19 & 2.43 & 1.66 & 0.14 & 0.52 & 3.84 & 25.1 & 0.37 & 8.4 & 11.5 \\
\hline WPSC096C & 0.78 & 0.18 & 0.05 & 0.73 & 0.12 & 4.58 & 6.3 & 2.06 & 1.82 & 0.18 & 0.47 & 2.97 & 27.3 & 0.4 & 8.7 & 8.8 \\
\hline WPSC $104 C$ & 0.65 & 0.24 & 0.07 & 0.58 & 0.07 & 6.05 & 4.99 & 1.68 & 1.27 & 0.12 & 0.25 & 3.43 & 24.8 & 0.28 & 11.4 & 7.0 \\
\hline WPSC108C & 1.55 & 0.38 & 0.1 & 1.45 & 0.22 & 3.06 & 10.4 & 1.32 & 1.14 & 0.11 & 0.43 & 4.48 & 21.1 & 0.23 & 5.8 & 14.5 \\
\hline WPSC112C & 0.92 & 0.22 & 0.06 & 0.86 & 0.14 & 4 & 7.37 & 1.75 & 1.52 & 0.08 & 0.87 & 3.21 & 25.7 & 0.35 & 7.6 & 10.3 \\
\hline WPSC116C & 1.28 & 0.66 & 0.18 & 1.10 & 0.31 & 1.69 & 22 & 1.03 & 0.6 & 0.05 & 0.49 & 9.64 & 10.8 & 0.14 & 3.2 & 30.8 \\
\hline WPSC122C & 1.7 & 0.18 & 0.05 & 1.65 & 0.2 & 4.47 & 4.99 & 2.1 & 1.78 & 0.16 & 0.74 & 2.07 & 24.1 & 0.36 & 8.4 & 7.0 \\
\hline WPSC133C & 4.33 & 0.53 & 0.14 & 4.19 & 0.5 & 3.44 & 15.8 & 1.39 & 1.39 & 0.21 & 0.2 & 6.71 & 16.3 & 0.25 & 6.5 & 22.1 \\
\hline WPSC133X & 4.4 & 0.46 & 0.13 & 4.27 & 0.48 & 4.11 & 15.1 & 1.54 & 1.69 & 0.25 & 0.23 & 6.33 & 19.4 & 0.3 & 7.8 & 21.1 \\
\hline WPSC135C & 3.69 & 0.95 & 0.26 & 3.43 & 0.41 & 2.01 & 24.7 & 0.92 & 0.76 & 0.19 & 0.12 & 10.7 & 8.27 & 0.14 & 3.8 & 34.6 \\
\hline WPSC137C & 2.53 & 0.89 & 0.24 & 2.29 & 0.29 & 2.54 & 23.3 & 1.42 & 0.94 & 0.27 & 0.13 & 10.1 & 11.1 & 0.18 & 4.8 & 32.6 \\
\hline WPSC138C & 1.47 & 1.21 & 0.33 & 1.14 & 0.16 & 1.1 & 33.7 & 0.52 & 0.33 & 0.12 & 0.11 & 14.8 & 4.36 & 0.06 & 2.1 & 47.1 \\
\hline WPSC $144 C$ & 1.32 & 0.22 & 0.06 & 1.26 & 0.15 & 5.58 & 6.37 & 2.36 & 1.98 & 0.41 & 0.43 & 2.72 & 28.5 & 0.42 & 10.5 & 8.9 \\
\hline WPSC146C & 0.22 & 0.15 & 0.04 & 0.18 & 0.05 & 0.25 & 4.35 & 0.12 & 0.06 & 0.02 & 0.05 & 1.85 & 38 & 0.01 & 0.5 & 6.1 \\
\hline WPSC15OC & 0.78 & 0.09 & 0.02 & 0.76 & 0.08 & 5.23 & 2.35 & 2.05 & 1.91 & 0.38 & 0.57 & 0.99 & 31.4 & 0.41 & 9.9 & 3.3 \\
\hline WPSC150X & 0.85 & 0.07 & 0.02 & 0.83 & 0.08 & 5.35 & 1.92 & 2.14 & 1.98 & 0.39 & 0.58 & 0.8 & 30.1 & 0.42 & 10.1 & 2.7 \\
\hline WPSC155C & 2.74 & 0.1 & 0.03 & 2.71 & 0.47 & 6.18 & 2.44 & 2.77 & 2.17 & 0.48 & 0.61 & 1.04 & 30.4 & 0.46 & 11.7 & 3.4 \\
\hline WPSC159C & 1.63 & 0.95 & 0.26 & 1.37 & 0.26 & 2.02 & 24 & 1.8 & 0.65 & 0.24 & 0.16 & 10.4 & 12.4 & 0.14 & 3.8 & 33.6 \\
\hline WPSC $170 C$ & 0.67 & 0.13 & 0.04 & 0.63 & 0.09 & 4.37 & 2.97 & 1.89 & 1.51 & 0.35 & 0.27 & 1.24 & 29.6 & 0.33 & 8.3 & 4.2 \\
\hline \multicolumn{17}{|l|}{ Section B } \\
\hline WPSB $170 \mathrm{C}$ & 3.01 & 0.13 & 0.04 & 2.97 & 1.34 & 1.73 & 2.71 & 0.87 & 0.48 & 0.16 & 0.08 & 1.18 & 31.1 & 0.09 & 3.3 & 3.8 \\
\hline
\end{tabular}

Table 1, Page 21 


\begin{tabular}{|c|c|c|c|c|c|c|c|c|c|c|c|c|c|c|}
\hline $\begin{array}{l}\text { Section C } \\
\text { Field No. }\end{array}$ & $\begin{array}{c}\text { FeOx, \%, } \\
\text { ICP-16 }\end{array}$ & $\begin{array}{c}\text { KOx, \%, ICP } \\
16\end{array}$ & $\begin{array}{c}\mathrm{MgOx}, \%, \\
\text { ICP-16 }\end{array}$ & $\begin{array}{c}\mathrm{NaOx}, \%, \\
10 \times-16\end{array}$ & $\begin{array}{c}\mathrm{POx}, \%, 1 \mathrm{CP} \\
16\end{array}$ & $\begin{array}{c}\text { SiOx, \%, } \\
\text { ICP-16 }\end{array}$ & $\begin{array}{l}\text { TIOx, \%, } \\
\text { ICP-16 }\end{array}$ & $\begin{array}{c}\text { Sum } \\
\text { Oxides }\end{array}$ & $\begin{array}{c}\text { Apatite } 211 \\
\text { peak ht. }\end{array}$ & $\begin{array}{c}\text { Apatite } 211 \text { peak } \\
\text { height, } \% \text { highest value, } \\
\text { both sections }\end{array}$ & $\begin{array}{c}\text { Ba, ppm, } \\
10 P-16\end{array}$ & $\begin{array}{c}\text { Cr, ppm, } \\
\text { ICp-16 }\end{array}$ & $\begin{array}{c}\text { Mn, ppm, } \\
\text { ICp-16 }\end{array}$ & $\begin{array}{c}\mathrm{Nb}, \mathrm{ppm} \text {, } \\
\mathrm{ICP}-16\end{array}$ \\
\hline WPSCO11C & 3.55 & 2.59 & 0.76 & 0.38 & 2.2 & 69.1 & 0.77 & 93.8 & 26 & $6 \%$ & 356 & 522 & 399 & 19 \\
\hline WPSCO15C & 0.79 & 0.39 & 0.27 & 0.92 & 32.5 & 9.9 & 0.10 & 93.4 & 171 & $37 \%$ & 157 & 684 & $<100$ & 14 \\
\hline WPSCO15X & 0.79 & 0.37 & 0.27 & 0.88 & 33.5 & 9.5 & 0.10 & 93.9 & & & 123 & 682 & $<100$ & $<10$ \\
\hline WPSCO2OC & 1.32 & 0.94 & 0.35 & 0.43 & 24.3 & 20.1 & 0.20 & 85.7 & 178 & $38 \%$ & 141 & 966 & 139 & $<10$ \\
\hline WPSCO28C & 0.82 & 0.48 & 0.23 & 0.26 & 30.7 & 10.9 & 0.10 & 88.2 & 191 & $41 \%$ & 95 & 818 & $<100$ & $<10$ \\
\hline WPSCO31C & 1.39 & 1.10 & 0.36 & 0.26 & 27.0 & 20.1 & 0.22 & 91.8 & 156 & $34 \%$ & 183 & 1210 & 155 & 12 \\
\hline WPSCO32C & 0.73 & 0.40 & 0.18 & 0.26 & 31.9 & 8.6 & 0.08 & 88.5 & 214 & $46 \%$ & 118 & 683 & 146 & $<10$ \\
\hline WPSCO35C & 1.82 & 1.45 & 0.35 & 0.27 & 18.5 & 30.6 & 0.37 & 84.3 & 132 & $28 \%$ & 178 & 677 & 102 & 14 \\
\hline WPSCO39C & 1.02 & 0.69 & 0.23 & 0.28 & 33.2 & 14.1 & 0.15 & 98.3 & 170 & $37 \%$ & 141 & 777 & $<100$ & $<10$ \\
\hline WPSCO41C & 1.43 & 1.01 & 0.27 & 0.27 & 28.2 & 19.8 & 0.22 & 93.5 & 166 & $36 \%$ & 150 & 644 & 255 & $<10$ \\
\hline WPSCO43C & 2.55 & 2.02 & 0.36 & 0.22 & 12.9 & 43.6 & 0.53 & 87.7 & 82 & $18 \%$ & 242 & 562 & 196 & $<10$ \\
\hline WPSCO $45 C$ & 1.30 & 0.96 & 0.28 & 0.27 & 25.7 & 20.3 & 0.23 & 88.4 & 175 & $38 \%$ & 144 & 835 & $<100$ & $<10$ \\
\hline WPSCOSOC & 2.50 & 2.37 & 0.36 & 0.30 & 14.9 & 39.6 & 0.43 & 89.1 & 122 & $26 \%$ & 219 & 1080 & 288 & $<10$ \\
\hline WPSCOSOX & 2.36 & 2.24 & 0.35 & 0.30 & 14.5 & 37.2 & 0.40 & 84.3 & & & 189 & 1010 & 270 & $<10$ \\
\hline WPSCO55C & 1.62 & 0.88 & 0.20 & 0.35 & 26.8 & 14.7 & 0.15 & 84.4 & 207 & $45 \%$ & 66 & 1100 & 109 & 13 \\
\hline WPSCO56C & 4.79 & 3.04 & 1.41 & 0.16 & 1.6 & 49.8 & 0.63 & 75.9 & 25 & $5 \%$ & 300 & 10510 & 171 & $<10$ \\
\hline WPSCO60C & 3.46 & 2.27 & 0.35 & 0.32 & 3.8 & 52.6 & 0.60 & 79.8 & 30 & $6 \%$ & 233 & 1350 & 582 & 11 \\
\hline WPSC063C & 3.05 & 0.92 & 0.15 & 0.28 & 22.6 & 21.6 & 0.27 & 82.1 & 199 & $43 \%$ & 153 & 536 & $<100$ & 11 \\
\hline WPSC063X & 3.32 & 1.01 & 0.18 & 0.32 & 27.0 & 22.9 & 0.28 & 94.4 & & & 216 & 687 & $<100$ & $<10$ \\
\hline WPSCO7OC & 3.09 & 1.81 & 0.33 & 0.42 & 8.2 & 45.6 & 0.52 & 78.6 & 84 & $18 \%$ & 268 & 1570 & $<100$ & $<10$ \\
\hline WPSCO75C & 3.13 & 2.59 & 0.27 & 0.36 & 4.1 & 57.1 & 0.73 & 82.3 & 35 & $8 \%$ & 305 & 553 & $<100$ & 14 \\
\hline WPSCO75X & 3.59 & 2.77 & 0.28 & 0.34 & 4.4 & 61.0 & 0.77 & 87.8 & & & 300 & 619 & $<100$ & $<10$ \\
\hline WPSCOBOC & 3.02 & 1.77 & 0.41 & 0.28 & 11.4 & 45.3 & 0.52 & 85.6 & 106 & $23 \%$ & 251 & 1560 & $<100$ & $<10$ \\
\hline WPSCO9OC & 3.02 & 2.14 & 0.32 & 0.40 & 8.8 & 53.7 & 0.63 & 89.2 & 100 & $22 \%$ & 310 & 1360 & $<100$ & $<10$ \\
\hline WPSCO91C & 3.47 & 2.00 & 0.23 & 0.70 & 8.8 & 53.7 & 0.62 & 89.3 & 76 & $16 \%$ & 270 & 986 & $<100$ & $<10$ \\
\hline WPSCO96C & 2.95 & 2.19 & 0.30 & 0.63 & 6.8 & 58.4 & 0.67 & 89.4 & 71 & $15 \%$ & 351 & 1400 & $<100$ & 12 \\
\hline WPSC104C & 2.40 & 1.53 & 0.20 & 0.34 & 7.9 & 53.0 & 0.47 & 84.3 & 44 & $9 \%$ & 562 & 848 & 220 & $<10$ \\
\hline WPSC108C & 1.89 & 1.37 & 0.18 & 0.58 & 10.3 & 45.1 & 0.38 & 80.1 & 106 & $23 \%$ & 167 & 1120 & $<100$ & $<10$ \\
\hline WPSC112C & 2.50 & 1.83 & 0.13 & 1.17 & 7.4 & 55.0 & 0.58 & 86.4 & 51 & $11 \%$ & 221 & 626 & 130 & 15 \\
\hline WPSC1 $16 C$ & 1.47 & 0.72 & 0.08 & 0.66 & 22.1 & 23.1 & 0.23 & 82.3 & 204 & $44 \%$ & 115 & 460 & $<100$ & $<10$ \\
\hline WPSC122C & 3.00 & 2.14 & 0.27 & 1.00 & 4.7 & 51.5 & 0.60 & 78.7 & 45 & $10 \%$ & 222 & 1370 & $<100$ & $<10$ \\
\hline WPSC133C & 1.99 & 1.67 & 0.35 & 0.27 & 15.4 & 34.9 & 0.42 & 83.5 & 112 & $24 \%$ & 240 & 2410 & $<100$ & $<10$ \\
\hline WPSC133X & 2.20 & 2.04 & 0.41 & 0.31 & 14.5 & 41.5 & 0.50 & 90.4 & & & 286 & 2710 & $<100$ & $<10$ \\
\hline WPSC135C & 1.32 & 0.92 & 0.32 & 0.16 & 24.5 & 17.7 & 0.23 & 83.5 & 163 & $35 \%$ & 157 & 1870 & $<100$ & $<10$ \\
\hline WPSC137C & 2.03 & 1.13 & 0.45 & 0.18 & 23.1 & 23.7 & 0.30 & 88.4 & 142 & $31 \%$ & 194 & 2570 & $<100$ & $<10$ \\
\hline WPSC $138 \mathrm{C}$ & 0.74 & 0.40 & 0.20 & 0.15 & 33.9 & 9.3 & 0.10 & 94.1 & 211 & $45 \%$ & 111 & 1050 & $<100$ & 11 \\
\hline WPSC1 $144 C$ & 3.37 & 2.39 & 0.68 & 0.58 & 6.2 & 61.0 & 0.70 & 94.4 & 66 & $14 \%$ & 351 & 1010 & $<100$ & 13 \\
\hline WPSC146C & 0.17 & 0.07 & 0.03 & 0.07 & 4.2 & 81.3 & 0.02 & 92.4 & 66 & $14 \%$ & 219 & 124 & $<100$ & $<10$ \\
\hline WPSC15OC & 2.93 & 2.30 & 0.63 & 0.77 & 2.3 & 67.2 & 0.68 & 89.9 & 36 & $8 \%$ & 316 & 633 & $<100$ & 11 \\
\hline WPSC1 $50 \mathrm{X}$ & 3.06 & 2.39 & 0.65 & 0.78 & 1.8 & 64.4 & 0.70 & 86.6 & & & 305 & 616 & $<100$ & 14 \\
\hline WPSC15SC & 3.96 & 2.61 & 0.80 & 0.82 & 2.4 & 65.0 & 0.77 & 91.5 & 40 & $9 \%$ & 347 & 852 & 262 & 15 \\
\hline WPSC159C & 2.57 & 0.78 & 0.40 & 0.22 & 23.8 & 26.5 & 0.23 & 92.0 & 97 & $21 \%$ & 390 & 953 & 2860 & $<10$ \\
\hline WPSC170C & 2.70 & 1.82 & 0.58 & 0.36 & 2.8 & 63.3 & 0.55 & 84.6 & 41 & $9 \%$ & 273 & 603 & 284 & $<10$ \\
\hline \multicolumn{15}{|l|}{ Section B } \\
\hline WPSB $170 C$ & 1.24 & 0.58 & 0.27 & 0.11 & 2.7 & 66.5 & 0.15 & 78.6 & & & 144 & 536 & $<100$ & $<10$ \\
\hline
\end{tabular}

Table 1, Page 22 


\begin{tabular}{|c|c|c|c|c|c|c|c|c|c|c|c|c|c|c|c|c|c|}
\hline $\begin{array}{l}\text { Section C } \\
\text { Field No. }\end{array}$ & $\begin{array}{l}\text { Sr, ppm, } \\
\text { ICP-16 }\end{array}$ & $\begin{array}{c}\text { Y, ppm, lCP } \\
16 \\
\end{array}$ & $\begin{array}{c}\mathrm{Zr}, \mathrm{ppm}, \\
\mathrm{ICP}-16\end{array}$ & $\begin{array}{c}\text { Al, } \%, I C P \\
40\end{array}$ & $\begin{array}{l}\text { Ca, \%, } \\
\text { ICP-40 }\end{array}$ & $\begin{array}{l}\mathrm{Fe}, \%, \\
\mathrm{ICP}-40\end{array}$ & $\begin{array}{c}K, \%, I C P- \\
40\end{array}$ & $\begin{array}{l}\text { Mg, \%, } \\
\text { ICP-40 }\end{array}$ & $\begin{array}{l}\mathrm{Na}, \%, \\
\mathrm{ICP}-40\end{array}$ & $\begin{array}{c}P, \%, I C P- \\
40\end{array}$ & $\begin{array}{c}\text { Ag, ppm, } \\
\text { ICP-40 }\end{array}$ & $\begin{array}{c}\text { Ba, ppm, } \\
\text { ICp-40 }\end{array}$ & $\begin{array}{c}\text { Be, ppm, } \\
\text { ICP-40 }\end{array}$ & $\begin{array}{c}\text { Bi, ppm, } \\
\text { ICP-40 }\end{array}$ & $\begin{array}{c}\text { Cd, ppm, } \\
\text { ICP-40 }\end{array}$ & $\begin{array}{c}\text { Ce, ppm, } \\
1 C P-40\end{array}$ & $\begin{array}{c}\text { Co, ppm, } \\
\text { ICP-40 }\end{array}$ \\
\hline WPSCO11C & 98 & 47 & 480 & 5.615 & 2.153 & 2.22 & 2.02 & 0.42 & 0.27 & 0.805 & $<2$ & 304 & $<1$ & $<50$ & 126 & 63 & 6 \\
\hline WPSCO15C & 985 & 137 & 104 & 1.09 & 28.8 & 0.42 & 0.33 & 0.147 & 0.69 & 13.3 & $<2$ & 107 & $<1$ & $<50$ & 118 & 13 & $<2$ \\
\hline WPSCO15X & 967 & 137 & 90 & 1.13 & 28.6 & 0.27 & 0.34 & 0.147 & 0.695 & 13.2 & $<2$ & 108 & $<1$ & $<50$ & 119 & 13 & $<2$ \\
\hline WPSCO2OC & 583 & 195 & 117 & 2.28 & 23.1 & 0.73 & 0.88 & 0.226 & 0.36 & 10.4 & 8 & 160 & $<1$ & $<50$ & 101 & 31 & $<2$ \\
\hline WPSCO28C & 581 & 116 & 75 & 1.305 & 29.4 & 0.59 & 0.48 & 0.147 & 0.23 & 14 & $<2$ & 113 & $<1$ & $<50$ & 104 & 8 & $<2$ \\
\hline WPSCO31C & 508 & 140 & 117 & 2.19 & 24.7 & 0.88 & 1.01 & 0.226 & 0.215 & 11.5 & $<2$ & 175 & $<1$ & $<50$ & 99 & 24 & $<2$ \\
\hline WPSCO32C & 572 & 183 & 65 & 1.035 & 29.1 & 0.49 & 0.37 & 0.116 & 0.215 & 13.8 & $<2$ & 93 & $<1$ & $<50$ & 93 & 19 & $<2$ \\
\hline WPSCO3SC & 382 & 133 & 211 & 3.21 & 18.6 & 1.24 & 1.43 & 0.215 & 0.245 & 8.335 & $<2$ & 207 & $<1$ & $<50$ & 85 & 44 & 2 \\
\hline WPSC039C & 624 & 198 & 113 & 1.31 & 28.3 & 0.36 & 0.6 & 0.131 & 0.21 & 13 & $<2$ & 109 & $<1$ & $<50$ & 105 & 20 & $<2$ \\
\hline WPSC041C & 521 & 208 & 123 & 2.195 & 24.8 & 0.89 & 0.95 & 0.163 & 0.205 & 11.5 & 2 & 119 & $<1$ & $<50$ & 86 & 37 & 4 \\
\hline WPSCO43C & 288 & 118 & 284 & 4.13 & 12.7 & 1.82 & 1.83 & 0.226 & 0.185 & 5.69 & 2 & 243 & $<1$ & $<50$ & 61 & 65 & 6 \\
\hline WPSCO45C & 549 & 158 & 156 & 2.02 & 23.5 & 0.64 & 0.91 & 0.173 & 0.225 & 10.8 & $<2$ & 136 & $<1$ & $<50$ & 76 & 25 & $<2$ \\
\hline WPSCOSOC & 545 & 198 & 189 & 4.455 & 13.3 & 1.72 & 2 & 0.221 & 0.23 & 6.155 & 11 & 206 & $<1$ & $<50$ & 69 & 56 & 5 \\
\hline WPSCOSOX & 515 & 185 & 184 & 4.495 & 13.8 & 1.75 & 2.11 & 0.221 & 0.24 & 6.29 & 10 & 206 & $<1$ & $<50$ & 69 & 54 & 4 \\
\hline WPSCOSSC & 1110 & 323 & 89 & 1.935 & 25.6 & 1.13 & 0.85 & 0.137 & 0.295 & 11.9 & 5 & 87 & $<1$ & $<50$ & 92 & so & $<2$ \\
\hline WPSCO56C & 161 & 61 & 297 & 6.21 & 2.357 & 3.27 & 2.55 & 0.856 & 0.12 & 0.64 & $<2$ & 298 & $<1$ & $<50$ & so & 39 & $<2$ \\
\hline WPSCO60C & 863 & 84 & 244 & 7.285 & 3.46 & 2.55 & 2.11 & 0.236 & 0.26 & 1.69 & 7 & 267 & $<1$ & $<50$ & 26 & 60 & 23 \\
\hline WPSC063C & 1430 & 199 & 149 & 2.515 & 21.8 & 1.1 & 0.94 & 0.095 & 0.25 & 9.63 & $<2$ & 171 & 2 & $<50$ & 40 & 60 & $<2$ \\
\hline WPSC063X & 1680 & 234 & 154 & 2.44 & 22.8 & 1.36 & 0.9 & 0.1 & 0.25 & 10.3 & $<2$ & 167 & 2 & $<50$ & 41 & 58 & $<2$ \\
\hline WPSCO70C & 1090 & 182 & 249 & 5.2 & 7.51 & 2.37 & 1.75 & 0.21 & 0.37 & 3.73 & 5 & 282 & 1 & $<50$ & 30 & 69 & $<2$ \\
\hline WPSCO75C & 304 & 64 & 386 & 5.735 & 3.229 & 2.37 & 2.38 & 0.168 & 0.305 & 1.895 & $<2$ & 312 & $<1$ & $<50$ & 18 & 72 & $<2$ \\
\hline WPSCO75X & 297 & 64 & 414 & 5.715 & 3.213 & 2.55 & 2.42 & 0.179 & 0.27 & 1.94 & 2 & 323 & $<1$ & $<50$ & 18 & 64 & $<2$ \\
\hline WPSCO80C & 684 & 251 & 303 & 4.54 & 10.4 & 2.14 & 1.63 & 0.257 & 0.23 & 4.885 & 4 & 251 & 1 & $<50$ & 35 & 84 & $<2$ \\
\hline WPSCO90C & 567 & 203 & 327 & 4.81 & 7.933 & 2.08 & 1.68 & 0.189 & 0.32 & 3.765 & 6 & 291 & 1 & $<50$ & 26 & 66 & $<2$ \\
\hline WPSCO91C & 537 & 180 & 352 & 4.63 & 7.77 & 2.39 & 1.62 & 0.137 & 0.545 & 3.815 & 5 & 257 & $<1$ & $<50$ & 23 & 62 & $<2$ \\
\hline WPSC096C & 592 & 135 & 387 & 4.99 & 5.996 & 2.05 & 1.88 & 0.184 & 0.49 & 2.785 & 7 & 332 & 1 & $<50$ & 19 & 58 & $<2$ \\
\hline WPSC104C & 5340 & 129 & 276 & 6.455 & 4.715 & 1.68 & 1.37 & 0.116 & 0.255 & 3.325 & 3 & 570 & 2 & $<50$ & 23 & 54 & 22 \\
\hline WPSC108C & 583 & 209 & 204 & 3.595 & 11.1 & 1.47 & 1.37 & 0.126 & 0.505 & 4.91 & 6 & 218 & 1 & $<50$ & 26 & 52 & 2 \\
\hline WPSC $112 C$ & 420 & 130 & 361 & 4.54 & 7.056 & 1.83 & 1.69 & 0.089 & 0.96 & 3.115 & 3 & 224 & $<1$ & $<50$ & 14 & 59 & 3 \\
\hline WPSC $116 C$ & 1360 & 267 & 200 & 2.02 & 22.2 & 1.11 & 0.74 & 0.058 & 0.59 & 10.2 & 3 & 127 & 1 & $<50$ & 20 & 60 & $<2$ \\
\hline WPSC122C & 314 & 143 & 313 & 5.225 & 5.408 & 2.33 & 2.09 & 0.173 & 0.865 & 2.33 & 8 & 290 & 1 & $<50$ & 36 & 57 & $<2$ \\
\hline WPSC133C & 779 & 199 & 213 & 3.485 & 14.8 & 1.29 & 1.41 & 0.2 & 0.2 & 6.54 & 15 & 209 & $<1$ & $<50$ & 149 & 37 & $<2$ \\
\hline WPSC133X & 788 & 213 & 261 & 3.98 & 13.1 & 1.4 & 1.61 & 0.231 & 0.22 & 5.675 & 15 & 245 & $<1$ & $<50$ & 147 & 43 & $<2$ \\
\hline WPSC135C & 932 & 138 & 117 & 2.245 & 23.3 & 0.79 & 0.85 & 0.189 & 0.135 & 10.7 & $<2$ & 161 & $<1$ & $<50$ & 219 & 19 & $<2$ \\
\hline WPSC137C & 690 & 210 & 172 & 2.785 & 21.9 & 1.07 & 1.02 & 0.278 & 0.135 & 9.27 & $<2$ & 185 & $<1$ & $<50$ & 165 & 34 & $<2$ \\
\hline WPSC $138 \mathrm{C}$ & 964 & 276 & 80 & 1.23 & 29.2 & 0.49 & 0.37 & 0.116 & 0.11 & 14.1 & $<2$ & 84 & $<1$ & $<50$ & 113 & 18 & $<2$ \\
\hline WPSC $144 C$ & 296 & 111 & 336 & 5.505 & 5.707 & 2.19 & 1.8 & 0.373 & 0.415 & 2.455 & 3 & 306 & $<1$ & $<50$ & 54 & 64 & 4 \\
\hline WPSC1 $146 C$ & 198 & 107 & $<10$ & 0.22 & 4.158 & 0.14 & 0.06 & 0.016 & 0.05 & 1.845 & $<2$ & 220 & $<1$ & $<50$ & 10 & 13 & $<2$ \\
\hline WPSC15OC & 122 & 93 & 344 & 5.46 & 2.247 & 2 & 1.94 & 0.378 & 0.585 & 0.935 & $<2$ & 294 & 1 & $<50$ & 30 & 58 & 3 \\
\hline WPSC15OX & 101 & 81 & 353 & 5.795 & 1.958 & 2.17 & 2.07 & 0.404 & 0.625 & 0.775 & $<2$ & 302 & 1 & $<50$ & 31 & 60 & 3 \\
\hline WPSC1SSC & 122 & 94 & 364 & 5.845 & 2.347 & 2.49 & 2.02 & 0.441 & 0.565 & 1.01 & $<2$ & 317 & $<1$ & $<50$ & 45 & 59 & $<2$ \\
\hline WPSC159C & 647 & 481 & 147 & 2.155 & 20.9 & 1.7 & 0.7 & 0.236 & 0.165 & 9.625 & 4 & 355 & $<1$ & $<50$ & 111 & 61 & 25 \\
\hline WPSC $170 \mathrm{C}$ & 120 & 104 & 265 & 4.775 & 3.092 & 2 & 1.67 & 0.368 & 0.295 & 1.315 & $<2$ & 292 & 1 & $<50$ & 16 & 52 & 4 \\
\hline \multicolumn{18}{|l|}{ Section B } \\
\hline WPSB $170 \mathrm{C}$ & 116 & 103 & 68 & 1.875 & 2.882 & 0.97 & 0.57 & 0.189 & 0.075 & 1.29 & $<2$ & 179 & $<1$ & $<50$ & 11 & 17 & $<2$ \\
\hline
\end{tabular}

Table 1, Page 23 


\begin{tabular}{|c|c|c|c|c|c|c|c|c|c|c|c|c|c|c|c|c|c|}
\hline $\begin{array}{l}\text { Section C } \\
\text { Field No. }\end{array}$ & $\begin{array}{c}\text { Cu, ppm, } \\
\text { ICP-40 }\end{array}$ & $\begin{array}{c}\text { Eu, ppm, } \\
\text { ICP-40 }\end{array}$ & $\begin{array}{l}\text { Ga, ppm, } \\
\text { ICP-40 }\end{array}$ & $\begin{array}{c}\text { Ho, ppm, } \\
\text { ICP-40 }\end{array}$ & $\begin{array}{l}\text { La, ppm, } \\
\text { ICP-40 }\end{array}$ & $\begin{array}{l}\text { Li, ppm, } \\
\text { ICP-40 }\end{array}$ & $\begin{array}{c}\text { Mn. ppm, } \\
\text { ICP-40 }\end{array}$ & $\begin{array}{c}\text { Mo, ppm, } \\
\text { ICP-40 }\end{array}$ & $\begin{array}{c}\text { Nb, ppm, } \\
\text { ICP-40 }\end{array}$ & $\begin{array}{c}\text { Nd, ppm, } \\
\text { ICP-40 }\end{array}$ & $\begin{array}{l}\text { Ni, ppm, } \\
\text { ICP-40 }\end{array}$ & $\begin{array}{c}\text { Pb, ppm, } \\
1 C P-40\end{array}$ & $\begin{array}{l}\text { Sc, ppm, } \\
1 C P-40\end{array}$ & $\begin{array}{l}\text { Sn, ppm, } \\
\text { ICP-40 }\end{array}$ & $\begin{array}{l}\mathrm{Sr}, \mathrm{ppm} \text {, } \\
\mathrm{ICP}-40\end{array}$ & $\begin{array}{l}\text { Th, ppm, } \\
\text { ICP-40 }\end{array}$ & $\begin{array}{l}\text { U, ppm, } \\
\text { ICp-40 }\end{array}$ \\
\hline WPSC011C & 71 & $<2$ & $<4$ & $<4$ & 35 & 27 & 345 & 72 & $<4$ & 29 & 244 & 10 & 8 & $<50$ & 82 & 12 & $<100$ \\
\hline WPSC015C & 99 & $<2$ & $<4$ & $<4$ & 96 & 11 & 39 & 19 & $<4$ & 29 & 55 & 12 & $<2$ & $<50$ & 880 & $<6$ & 115 \\
\hline WPSCO15X & 105 & $<2$ & $<4$ & $<4$ & 99 & 12 & 38 & 20 & $<4$ & 34 & 55 & 15 & $<2$ & $<50$ & 873 & $<6$ & 137 \\
\hline WPSCO2OC & 88 & 2 & $<4$ & 5 & 166 & 21 & 118 & 37 & $<4$ & 63 & 114 & 11 & $<2$ & $<50$ & 572 & $<6$ & $<100$ \\
\hline WPSCO28C & 72 & $<2$ & $<4$ & $<4$ & 88 & 14 & 87 & 32 & $<4$ & 30 & 68 & 10 & 3 & $<50$ & 580 & $<6$ & 120 \\
\hline WPSCO31C & 90 & 2 & $<4$ & 4 & 106 & 22 & 150 & 40 & $<4$ & 42 & 93 & 15 & 2 & $<50$ & 492 & $<6$ & 130 \\
\hline WPSCO32C & 59 & 2 & $<4$ & $<4$ & 134 & 11 & 147 & 18 & $<4$ & 44 & 63 & 10 & 3 & $<50$ & 552 & $<6$ & 125 \\
\hline WPSCO35C & 67 & 2 & $<4$ & 4 & 109 & 19 & 95 & 16 & $<4$ & 51 & 124 & 10 & $<2$ & $<50$ & 387 & $<6$ & $<100$ \\
\hline WPSC039C & 67 & 3 & $<4$ & 4 & 136 & 13 & 28 & 6 & $<4$ & 56 & 65 & 13 & $<2$ & $<50$ & 560 & $<6$ & $<100$ \\
\hline WPSC041C & 65 & 3 & $<4$ & 4 & 158 & 13 & 234 & 19 & $<4$ & 65 & 118 & 10 & 2 & $<50$ & 497 & $<6$ & $<100$ \\
\hline WPSCO43C & 61 & 3 & $<4$ & $<4$ & 97 & 20 & 181 & 27 & $<4$ & 52 & 180 & 13 & 8 & $<50$ & 273 & 8 & $<100$ \\
\hline WPSCO45C & 65 & 2 & $<4$ & $<4$ & 115 & 17 & 48 & 12 & $<4$ & 52 & 74 & 12 & $<2$ & $<50$ & 519 & $<6$ & 127 \\
\hline WPSCOSOC & 89 & 4 & $<4$ & 5 & 159 & 21 & 293 & 23 & $<4$ & 77 & 190 & 9 & 9 & $<50$ & 503 & $<6$ & $<100$ \\
\hline WPSCOSOX & 92 & 4 & $<4$ & 4 & 162 & 21 & 266 & 24 & $<4$ & 79 & 190 & 11 & 9 & $<50$ & 527 & $<6$ & $<100$ \\
\hline WPSCO55C & 147 & 6 & $<4$ & 8 & 270 & 14 & 98 & 47 & $<4$ & 149 & 109 & 10 & 5 & $<50$ & 1140 & $<6$ & $<100$ \\
\hline WPSC056C & 979 & 4 & $<4$ & $<4$ & 52 & 79 & 146 & 192 & $<4$ & 57 & 235 & 70 & 17 & $<50$ & 154 & 20 & $<100$ \\
\hline WPSC060C & 100 & $<2$ & $<4$ & $<4$ & 97 & 26 & 598 & 16 & $<4$ & 51 & 223 & 8 & 12 & $<50$ & 868 & 9 & $<100$ \\
\hline WPSCO63C & 44 & 5 & $<4$ & 5 & 203 & 10 & 8 & 7 & $<4$ & 122 & 27 & 7 & $<2$ & $<50$ & 1520 & $<6$ & $<100$ \\
\hline WPSC063x & 47 & 5 & $<4$ & 6 & 206 & 11 & 11 & 6 & $<4$ & 124 & 26 & 6 & $<2$ & $<50$ & 1530 & $<6$ & $<100$ \\
\hline WPSC07OC & 112 & 4 & $<4$ & 4 & 167 & 26 & 58 & 13 & $<4$ & 89 & 97 & 9 & 10 & $<50$ & 1090 & 7 & $<100$ \\
\hline WPSC075C & 45 & 2 & $<4$ & $<4$ & 64 & 17 & $<4$ & 9 & $<4$ & 54 & 50 & 11 & 11 & $<50$ & 306 & 15 & $<100$ \\
\hline WPSCO75X & 49 & 3 & 6 & $<4$ & 64 & 17 & 4 & 10 & $<4$ & 48 & 49 & 10 & 12 & $<50$ & 285 & 13 & $<100$ \\
\hline WPSCOBOC & 144 & 5 & $<4$ & 6 & 185 & 25 & 30 & 9 & $<4$ & 116 & 62 & 10 & 10 & $<50$ & 665 & 8 & $<100$ \\
\hline WPSCO9OC & 99 & 4 & 6 & 5 & 139 & 21 & 19 & 11 & $<4$ & 91 & 52 & 10 & 10 & $<50$ & 530 & 7 & $<100$ \\
\hline WPSCO91C & 85 & 4 & $<4$ & 5 & 115 & 16 & 91 & 15 & $<4$ & 74 & 79 & 10 & 10 & $<50$ & 499 & 8 & $<100$ \\
\hline WPSC096C & 107 & 3 & 4 & $<4$ & 92 & 21 & 48 & 12 & $<4$ & 58 & 62 & 10 & 10 & $<50$ & 567 & 7 & $<100$ \\
\hline WPSC104C & 68 & 3 & $<4$ & $<4$ & 102 & 13 & 187 & 7 & $<4$ & 58 & 214 & $<4$ & 8 & $<50$ & $<2$ & 6 & $<100$ \\
\hline WPSC108C & 127 & 4 & 4 & 6 & 146 & 19 & 51 & 27 & $<4$ & 87 & 145 & 5 & 9 & $<50$ & 618 & 8 & $<100$ \\
\hline WPSC112C & 71 & 3 & $<4$ & 4 & 87 & 10 & 122 & 25 & $<4$ & 62 & 133 & 7 & 8 & $<50$ & 403 & $<6$ & $<100$ \\
\hline WPSC116C & 57 & 6 & $<4$ & 7 & 200 & 7 & 71 & 20 & $<4$ & 131 & 90 & 7 & 5 & $<50$ & 1390 & $<6$ & $<100$ \\
\hline WPSC122C & 128 & 3 & $<4$ & $<4$ & 106 & 20 & 10 & 29 & $<4$ & 62 & 185 & 8 & 10 & $<50$ & 333 & 8 & $<100$ \\
\hline WPSC133C & 207 & 3 & $<4$ & $<4$ & 120 & 20 & 5 & 5 & $<4$ & 60 & 154 & 10 & 5 & $<50$ & 730 & $<6$ & $<100$ \\
\hline WPSC133X & 219 & 3 & 5 & $<4$ & 117 & 23 & 4 & 6 & $<4$ & 65 & 169 & 14 & 8 & $<50$ & 674 & $<6$ & $<100$ \\
\hline WPSC135C & 189 & 2 & $<4$ & $<4$ & 85 & 14 & 10 & 21 & $<4$ & 42 & 152 & 14 & $<2$ & $<50$ & 903 & $<6$ & 139 \\
\hline WPSC137C & 230 & 3 & $<4$ & 5 & 126 & 22 & 17 & 46 & $<4$ & 62 & 194 & 17 & 2 & $<50$ & 673 & $<6$ & 148 \\
\hline WPSC138C & 85 & 3 & $<4$ & 5 & 158 & 8 & 74 & 18 & $<4$ & 76 & 71 & 11 & 3 & $<50$ & 887 & $<6$ & $<100$ \\
\hline WPSC144C & 66 & 3 & 8 & $<4$ & 81 & 30 & 59 & 8 & $<4$ & 62 & 111 & 12 & 9 & $<50$ & 257 & 9 & $<100$ \\
\hline WPSC146C & 15 & 2 & $<4$ & $<4$ & 85 & 4 & 77 & 2 & $<4$ & 63 & 12 & $<4$ & $<2$ & $<50$ & 182 & $<6$ & $<100$ \\
\hline WPSC15OC & 42 & 3 & 7 & $<4$ & 70 & 24 & 42 & 8 & $<4$ & 56 & 126 & 8 & 9 & $<50$ & 110 & 11 & $<100$ \\
\hline WPSC150X & 45 & 3 & $<4$ & $<4$ & 65 & 26 & 48 & 9 & $<4$ & 50 & 141 & 7 & 10 & $<50$ & 98 & 10 & $<100$ \\
\hline WPSC155C & 41 & 2 & 8 & $<4$ & 64 & 27 & 197 & 7 & $<4$ & 45 & 144 & 10 & 10 & $<50$ & 107 & 11 & $<100$ \\
\hline WPSC159C & 57 & 8 & $<4$ & 9 & 309 & 18 & 2680 & 14 & $<4$ & 181 & 493 & 14 & 7 & $<50$ & 588 & $<6$ & $<100$ \\
\hline WPSC170C & 41 & 3 & $<4$ & 4 & 78 & 24 & 284 & 10 & $<4$ & 60 & 140 & 9 & 9 & $<50$ & 116 & 7 & $<100$ \\
\hline \multicolumn{18}{|l|}{ Section B } \\
\hline WPSB $170 \mathrm{C}$ & 52 & 3 & $<4$ & 4 & 70 & 18 & 46 & 23 & $<4$ & 49 & 179 & $<4$ & 4 & $<50$ & 121 & $<6$ & $<100$ \\
\hline
\end{tabular}

Table 1, Page 24 
Section C (wpsC) Sample Geochemistry

\begin{tabular}{|c|c|c|c|c|c|}
\hline $\begin{array}{l}\text { Section C } \\
\text { Field No. }\end{array}$ & $\begin{array}{l}\text { V, ppm, } \\
10 P-40\end{array}$ & $\begin{array}{l}\text { Y, ppm, } \\
\text { ICP-40 }\end{array}$ & $\begin{array}{c}\text { Yb, ppm, } \\
1 C p-40\end{array}$ & $\begin{array}{c}\text { Zn, ppm, } \\
\text { ICP-40 }\end{array}$ & $\begin{array}{l}\text { eU, un-scaled, ppm, } \\
\text { channel interval avg. }\end{array}$ \\
\hline WPSCOI1C & 692 & 30 & 3 & 2440 & 58 \\
\hline WPSC015C & 1160 & 129 & 6 & 991 & 167 \\
\hline WPSC015X & 1180 & 131 & 6 & 1020 & \\
\hline WPSCO2OC & 555 & 203 & 9 & 1130 & 127 \\
\hline WPSCO28C & 1560 & 125 & 6 & 833 & 187 \\
\hline WPSCO31C & 1680 & 144 & 7 & 1060 & 156 \\
\hline WPSCO32C & 1120 & 187 & 9 & 824 & 176 \\
\hline WPSCO35C & 1320 & 147 & 8 & 1230 & 157 \\
\hline WPSC039C & 1450 & 187 & 9 & 818 & 158 \\
\hline WPSCO41C & 922 & 209 & 10 & 1000 & 166 \\
\hline WPSCO43C & 909 & 122 & 7 & 1660 & 177 \\
\hline WPSCO45C & 1660 & 161 & 8 & 894 & 151 \\
\hline WPSCO5OC & 400 & 199 & 10 & 1240 & 104 \\
\hline WPSCOSOX & 414 & 203 & 10 & 1240 & \\
\hline WPSCOS5C & 516 & 355 & 16 & 672 & 113 \\
\hline WPSC056C & 8280 & 55 & 9 & 1230 & 113 \\
\hline WPSC060C & 407 & 88 & 6 & 1200 & 48 \\
\hline WPSC063C & 119 & 224 & 10 & 520 & 39 \\
\hline WPSC063X & 127 & 232 & 10 & 515 & \\
\hline WPSCO7OC & 301 & 196 & 10 & 495 & 35 \\
\hline WPSCO75C & 223 & 66 & 5 & 229 & 28 \\
\hline WPSCO75X & 266 & 66 & 5 & 233 & \\
\hline WPSC080C & 231 & 255 & 13 & 386 & 41 \\
\hline WPSCO9OC & 181 & 201 & 10 & 276 & 40 \\
\hline WPSCO91C & 190 & 174 & 10 & 403 & 41 \\
\hline WPSC096C & 196 & 134 & 8 & 321 & 40 \\
\hline WPSC104C & 234 & 131 & 7 & 1380 & 45 \\
\hline WPSC108C & 195 & 235 & 13 & 713 & 43 \\
\hline WPSC112C & 184 & 132 & 8 & 692 & 39 \\
\hline WPSC116C & 126 & 291 & 14 & 480 & 46 \\
\hline WPSC122C & 258 & 160 & 9 & 850 & 54 \\
\hline WPSC133C & 861 & 202 & 10 & 641 & 90 \\
\hline WPSC1 $133 X$ & 955 & 194 & 10 & 717 & \\
\hline WPSC135C & 2160 & 142 & 8 & 952 & 141 \\
\hline WPSC137C & 2200 & 209 & 12 & 1370 & 169: \\
\hline WPSC $138 \mathrm{C}$ & 852 & 273 & 13 & 700 & 165 \\
\hline WPSC144C & 402 & 98 & 6 & 635 & 70 \\
\hline WPSC146C & 36 & 104 & 4 & 62 & 53 \\
\hline WPSC15OC & 228 & 89 & 5 & 669 & 52 \\
\hline WPSC150X & 231 & 79 & 5 & 748 & \\
\hline WPSC155C & 400 & 88 & 7 & 519 & 44 \\
\hline WPSC159C & 454 & 461 & 23 & 1140 & 73 \\
\hline WPSC170C & 203 & 106 & 7 & 610 & 25 \\
\hline \multicolumn{6}{|l|}{ Section B } \\
\hline WPSB $170 C$ & 176 & 111 & 6 & 826 & \\
\hline
\end{tabular}

Table 1, Page 25 


\begin{tabular}{|c|c|c|c|c|c|c|c|c|c|c|c|c|c|c|c|c|c|c|c|}
\hline Fiveld number & Lab No. & Unt within Meade Peak Member & Lithoiogy & $\begin{array}{l}\text { Intervat } \\
\text { base, } f t\end{array}$ & $\begin{array}{l}\text { Interval } \\
\text { top, ft }\end{array}$ & $\begin{array}{c}\text { Interval } \\
\text { thrckness, ft }\end{array}$ & $\begin{array}{c}\text { Interval } \\
\text { madpount, it }\end{array}$ & $\begin{array}{l}\text { As, ppm, } \\
\text { Hydr. AA }\end{array}$ & $\begin{array}{l}\text { Se, ppm } \\
\text { Hydr. AA }\end{array}$ & $\begin{array}{l}S D_{1}, \mathrm{ppm}_{-} \\
\text {Hydr. AA }\end{array}$ & $\begin{array}{l}\text { Te, ppm, } \\
\text { Mydr. AA }\end{array}$ & $\begin{array}{l}\text { Th, ppm, } \\
\text { Hydr. AA }\end{array}$ & $\begin{array}{c}\text { CO2, \%, } \\
\text { aciditication }\end{array}$ & $\begin{array}{c}\text { Carbonate } \mathrm{C} \text {. } \\
\text { \%, } \\
\text { acrification }\end{array}$ & $\begin{array}{c}C_{1} \% . \\
\text { combustion }\end{array}$ & $\begin{array}{c}\text { Organcic } C, \%, \\
\text { difference }\end{array}$ & $\begin{array}{c}\text { S, \%, } \\
\text { combustion }\end{array}$ & $\begin{array}{l}\text { Hg. ppm, } \\
\text { CVAA }\end{array}$ & $\begin{array}{c}A 1, \%, 16 \\
16\end{array}$ \\
\hline WPSDO0OC & C-136272 & Top of Grandeur & limestone & 0.00 & 0.00 & 0.00 & 0.00 & 4.3 & 1.0 & $<0.6$ & $<0.1$ & 0.3 & 43.3 & 11.8 & 11.9 & \begin{tabular}{|l|}
0.1 \\
\end{tabular} & $<0.05$ & $<0.02$ & \begin{tabular}{|l|l|}
0.22 \\
\end{tabular} \\
\hline WPSO0000x & $c-136278$ & & duphicate of previon & s semple & & & & 3.4 & 1.2 & $<0.6$ & $<0.1$ & 0.6 & 43.5 & 11.9 & 12.0 & 0.1 & $<0.05$ & $<0.02$ & 0.23 \\
\hline WPSO0.5C & C-136238 & Lower Ore Unit, Fist-sede Bed & phosphonte & 0.00 & 0.50 & 0.50 & 0.25 & 12.8 & 1.4 & 1.1 & $<0.1$ & 0.7 & 3.8 & 1.0 & 1.3 & 0.2 & 0.65 & 0.07 & 0.35 \\
\hline WPSDO006C & C-136262 & Lower Ore Unt, Footwall Mudstone & dolomite & 5.00 & 7.80 & 2.80 & 6.40 & 14.3 & 2.6 & 2.6 & $<0.1$ & 2.8 & 27.0 & 7.4 & 7.8 & 0.5 & 0.07 & 0.08 & 2.18 \\
\hline WPSDOOOC & C-136288 & Lower Ore Unt, footwall Mudstone & red mudstone & 7.80 & 10.50 & 2.70 & 9.15 & 47.2 & 20.0 & 14.0 & 0.2 & 11.8 & 1.0 & 0.3 & 2.1 & 1.8 & 0.63 & 0.56 & 3.42 \\
\hline WPSDO014C & $c-136274$ & Lower Ore Unit, A-Bed Ore & phosphorite & 10.50 & 14.70 & 4.20 & 12.60 & 15.2 & 10.6 & 5.0 & $<0.1$ & 3.4 & 2.1 & 0.6 & 2.0 & 1.4 & 1.01 & 0.40 & 0.45 \\
\hline WPSDO16C & $c-136251$ & Lower Ore Unt, A Cap & phosphonte & 14.70 & 17.00 & 2.30 & 15.85 & 12.0 & 6.1 & 2.3 & $<0.1$ & 1.3 & 19.7 & 5.4 & 6.4 & 1.0 & 0.32 & 0.19 & 0.81 \\
\hline WPSDO018C & $c-136232$ & Lower Ore Unit, A Cap & dociomite & 17.00 & 19.80 & 2.80 & 18.40 & 14.5 & 11.3 & 4.1 & $<0.1$ & 1.8 & 19.3 & 5.3 & 6.6 & 1.4 & 0.29 & 0.25 & 1.58 \\
\hline WPSDOZ20C & c-136269 & Lower Ore Unt, Lower B Ore & dolomite & 19.80 & 21.00 & 1.20 & 20.40 & 19.8 & 8.9 & 4.1 & $<0.1$ & 1.7 & 16.1 & 4.4 & 5.8 & 1.4 & 0.30 & 0.19 & 1.59 \\
\hline WPSDO23C & $c-136240$ & Lower Ore Unt, Lower B Ore & phosphonte & 21.00 & 25.00 & 4,00 & 23.00 & 13.3 & 105.0 & 3.6 & 0.1 & 1.4 & 6.5 & 1.8 & 3.2 & 1.4 & 0.43 & 0.21 & 1.07 \\
\hline WPSDO25C & $C-136263$ & Lower Ore Unt, B Hudstone & Iimestone & 25.00 & 26.00 & 1.00 & 25.50 & 17.7 & 5.1 & 2.7 & $<0.1$ & 1.2 & 28.0 & 7.6 & 8.0 & 0.4 & 0.08 & 0.10 & 2.21 \\
\hline WPS0027C & $c-136256$ & Lowver Ore Unit, Upper B Ore & phosphonite & 26.00 & 29.00 & 3.00 & 27.50 & 24.4 & 7.7 & 4.2 & $<.1$ & 1.6 & 1.3 & 0.4 & 2.0 & 1.6 & 0.41 & 0.35 & 1.51 \\
\hline WPSD0027x & C-136228 & 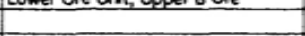 & duplicate of previon & 3 sample & 20.00 & 2.00 & $2 \pi .50$ & 24.1 & 6.0 & 5.1 & 0.1 & 1.4 & 1.2 & 0.3 & 1.9 & 1.5 & 0.40 & 0.33 & 1.72 \\
\hline WPSDO030C & C. -136264 & Lower Ore Unit, Upper B Ore & phosphonte & 29.00 & 31.00 & 2.00 & 30.00 & 17.8 & 5.1 & 3.7 & $<.1$ & 0.9 & 4.5 & 1.2 & 3.4 & 2.1 & 0.47 & 0.26 & 1.34 \\
\hline WPSDO31C & $c-136273$ & Lower Ore Unit, False Cap & dolomite & 31.00 & 32.00 & 1.00 & 31.50 & 14.0 & 2.4 & 2.1 & $<0.1$ & 0.2 & 36.0 & 9.8 & 9.9 & 0.1 & $<0.05$ & 0.08 & 1.56 \\
\hline WPSDO33C & C-136249 & Lower Ore Unt, False Cap & & 32.00 & 34.80 & 2.80 & 33.40 & 11.1 & 3.2 & 1.8 & $<0.1$ & $<0.1$ & 36.0 & 9.8 & 10.4 & 0.6 & 0.11 & 0.11 & 1.16 \\
\hline WPSD0036C & C. -136250 & Lower Ore Unit, False Cap & & 34.80 & 36.50 & 1.70 & 35.65 & 40.1 & 325.0 & 11.2 & 0.2 & 1.8 & 1.8 & 0.5 & 5.4 & 4.9 & 0.85 & 0.59 & 5.06 \\
\hline WPSDO039C & $C-136267$ & Lower Ore Unit, False Cap & dolomite & 36.50 & 41.00 & 4.50 & 38.75 & 15.1 & 13.1 & 1.3 & $<0.1$ & $<0.1$ & 31.9 & 8.7 & 10.1 & 1.4 & 0.21 & 0.17 & 1.12 \\
\hline WPSDO041C & $C-136266$ & Lower Ore Unit, C Bed & phosphonte & 41.00 & 42.00 & 1.00 & 41.50 & 40.3 & 14.9 & 6.3 & $\infty .1$ & 0.2 & 2.4 & 0.7 & 4.6 & 4.0 & 0.94 & 0.41 & 0.87 \\
\hline WPSDO43C & C-136233 & Lower Ore Unit, C Bed & phosphonte & 42.00 & 43.75 & 1.75 & 42.88 & 66.4 & 361.0 & 45.1 & 0.2 & 6.0 & 2.7 & 0.7 & 25.0 & 24.3 & 4.86 & 2.73 & -3.05 \\
\hline WPSDO046C & $c .136261$ & Moddle Waste & & 43.75 & 48.00 & 4.25 & 45.88 & 34.8 & 78.9 & 7.2 & 0.1 & 0.6 & 7.7 & 2.1 & 11.8 & 9.7 & 3.01 & 0.68 & 3.85 \\
\hline WPSDO049C & C-136239 & Moddle Waste & & 48.00 & 50.00 & 2.00 & 49.00 & 36.8 & 9.3 & 7.7 & $<0.1$ & 0.5 & 6.6 & 1.8 & 10.8 & 9.0 & 3.01 & 0.57 & 4.34 \\
\hline WPSDOS2C & $c-136237$ & Middle Waste & \begin{tabular}{|l|l|l|l|l|l|l|} 
silstone \\
\end{tabular} & 50.00 & 54.00 & 4.00 & 52.00 & 23.5 & 68.4 & 4.1 & $<0.1$ & 0.7 & 15.3 & 4.2 & 11.4 & 7.2 & 2.31 & 0.40 & 2.94 \\
\hline WPSDOSS2x & $c-136255$ & & duphicate of previou & s sample & & & & 27.1 & 64.8 & 3.6 & $<0.1$ & 0.4 & 15.3 & 4.2 & 11.0 & 6.8 & 2.24 & 0.36 & 2.89 \\
\hline WPSDOOSSC & $c-136242$ & Maddle Waste & 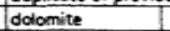 & 54.00 & 55.25 & 1.25 & 54.63 & 92 & 12.8 & 1.0 & $<0.1$ & 0.2 & 38.0 & 10.4 & 11.5 & 1.1 & 0.57 & 0.07 & 1.11 \\
\hline wPSDossx & $c .136224$ & & dupleste of previox & s semple & & & & 9.5 & 12.9 & 1.3 & $<0.1$ & 0.2 & 38.4 & 10.5 & 11.2 & 0.7 & 0.55 & 0.11 & 1.10 \\
\hline WPSDOSEC & $c-136275$ & Middle Waste & & 55.25 & 61.00 & 5.75 & 58.13 & 29.1 & 66.9 & 5.0 & $<0.1$ & 0.4 & 5.2 & 1.4 & 8.6 & 7.2 & 2.89 & 0.52 & 3.85 \\
\hline WPSD0062C & $c-136281$ & Middle Waste & dolamite & 61.00 & 63.00 & 2.00 & 62.00 & 6.1 & 10.2 & 0.6 & $<0.1$ & $<0.1$ & 41.9 & 11.4 & 11.6 & 0.2 & 0.42 & 0.08 & 0.79 \\
\hline WPS0067C & $c-136223$ & Middle Waste & & 63.00 & 69.50 & 6.50 & 66.25 & 27.1 & 59.0 & 5.6 & $<0.1$ & 0.6 & 5.3 & 1.4 & 8.9 & 7.4 & 3.15 & 0.34 & 4.76 \\
\hline WPSD0067x & $c .136279$ & & ouphencte of previou & is semple & & & & 30.0 & 74.5 & 5.7 & 0.1 & 0.4 & 5.7 & 1.6 & 8.9 & 7.3 & 3.13 & 0.37 & 4.49 \\
\hline WPSDOTIC & $c-136234$ & Middle Waste & & 69.50 & 72.00 & 2.50 & 70.75 & 25.5 & 52.5 & 4.5 & $<0.1$ & 0.4 & 2.2 & 0.6 & 6.5 & 5.9 & 3.18 & 0.26 & 5.28 \\
\hline WPSDO7SC & $C-136226$ & Middle Waste & & 72.00 & 79.00 & 7.00 & 75.50 & 30.7 & 91.2 & 5.3 & $<0.1$ & 0.8 & 2.2 & 0.6 & 7.9 & 7.3 & 3.23 & 0.34 & 5.40 \\
\hline WPSDOBOC & C-136286 & Middle Waste & & 79.00 & 80.00 & 1.00 & 79.50 & 26.0 & 50.0 & 4.8 & $<0.1$ & 0.4 & 1.9 & 0.5 & 6.6 & 6.1 & 3.58 & 0.25 & 5.17 \\
\hline WPSDOOSAC & C-136283 & Middle Waste & dolomite & 80.00 & 87.00 & 7.00 & 83.50 & 11.4 & 13.9 & 1.7 & $<0.1$ & 0.2 & 34.0 & 9.3 & 11.4 & 2.1 & 0.88 & 0.10 & 1.58 \\
\hline WPSDOB7C & $c-136276$ & Middle Waste & & 87.00 & 87.50 & 0.50 & 87.25 & 16.6 & 30.4 & 2.4 & $<0.1$ & $<0.1$ & 30.1 & 8.2 & 12.1 & 3.9 & 1.59 & 0.19 & 2.28 \\
\hline WPSDO093C & $c-136257$ & Middle Waste & & 87.50 & 96.30 & 8.80 & 91.90 & 35.7 & 209.0 & 8.4 & 0.1 & 0.8 & 0.4 & 0.1 & 13.7 & 13.6 & 3.86 & 0.81 & 4.01 \\
\hline WPSDO09BC & $c-136246$ & Moddle Weste & phosphonte & 96.30 & 100.00 & 3.70 & 98.15 & 32.9 & 193.0 & 8.7 & $<0.1$ & 1.5 & 0.6 & 0.2 & 19.1 & 18.9 & 4.02 & 0.82 & 2.61 \\
\hline WPSD0098X & C-136268 & & duphecate of previoi & is sample & & & & 33.5 & 218.0 & 9.4 & 0.2 & 1.2 & 0.5 & 0.1 & 19.3 & 19.2 & 4.56 & 0.82 & 3.06 \\
\hline WPSO101C & $c-136236$ & Middle Waste & & 100.00 & 102.00 & 2.00 & 101.00 & 31.4 & 156.0 & 5.6 & 0.1 & 1.4 & 0.2 & 0.1 & 4.4 & 4.3 & 3.19 & 0.47 & 5.27 \\
\hline WPSO101x & C-136243 & & dupicate of previon & is sample & & & & 31.3 & 115.0 & 5.0 & $<0.1$ & 1.2 & 0.2 & 0.1 & 4.2 & 4.1 & 3.09 & 0.44 & 5.20 \\
\hline WPSD102C & C-136285 & Middle Waste & carbon seam & 102.00 & 102.50 & 0.50 & 10225 & 16.5 & 80.6 & 3.8 & 0.1 & 0.8 & 0.9 & 0.2 & 10.8 & 10.6 & 2.43 & 0.47 & 1.71 \\
\hline WPSD $103 c$ & $\frac{1-136203}{c-136231}$ & Middle Waste & caroon seam & $\frac{102.50}{102.50}$ & $\frac{10<.30}{104.00}$ & $\frac{1.50}{1.50}$ & $\frac{10<25}{103.25}$ & 30.8 & 110.0 & $\frac{3.0}{5.5}$ & 0.1 & 0.9 & 0.1 & 0.0 & $\frac{0.6}{2.5}$ & $\frac{10.0}{2.5}$ & 3.09 & 0.35 & 5.39 \\
\hline WPSO $104 C$ & c. 136270 & Middle Waste & carbon seam & 104.00 & 104.50 & 0.50 & 104.25 & 31.8 & 139.0 & 4.4 & 0.1 & 0.9 & 0.6 & 0.2 & 9.4 & 9.3 & 3.80 & 0.54 & 3.63 \\
\hline WoSD104x & $c-136230$ & & dupicate of previo & is sample & & & & 28.7 & 116.0 & 4.2 & 0.2 & 0.5 & 0.6 & 0. & 9.4 & 9 & 3. & 0.52 & 3.59 \\
\hline WPSO10BC & C. 136259 & Middie Waste & & 104.50 & 108.75 & 4.25 & 106.63 & 32.5 & 76.7 & 2.4 & $<0.1$ & 1.3 & 0.3 & 0.1 & 4.3 & 4.3 & 3.7 & 0.4 & 4.66 \\
\hline WPSD109C & c-136271 & Middle Waste & & 108.75 & 109.25 & 0.50 & 109.00 & 29.8 & 33.4 & 2.1 & $<0.1$ & 0.8 & 0.4 & 0.1 & 2.5 & 2.3 & 3.33 & 0.51 & 3.98 \\
\hline WPSO $114 C$ & C. -136287 & Muddle Waste & & 109.25 & 117.50 & 8.25 & 113.38 & 31.8 & 39.9 & 2.3 & 60.1 & & 0.9 & 0.3 & 3.5 & 3.2 & 3 & 0.43 & 5.75 \\
\hline WPSO120C & $C-136282$ & Middie Wraste & dolomite & 117.50 & 125.50 & 8.00 & 121.50 & 11.6 & 12.6 & 1.0 & $<0.1$ & 0.2 & 26.8 & 7.3 & 8.3 & 0.9 & 1.30 & 0.14 & 2.40 \\
\hline WPSO126C & C-136235 & Middle Waste & carbon seam & 125.50 & 126.00 & 0.50 & 125.75 & 41.5 & 37.7 & 2.0 & $<0.1$ & 2.0 & 0.8 & 0.2 & 5.4 & 5.1 & 4.01 & 0.61 & 2.67 \\
\hline WPSD1 $130 C$ & C-136254 & Middle Wrste & & 126.00 & 131.50 & 5.50 & 128.75 & 24.7 & 26.1 & 1.6 & $<0.1$ & 0.7 & 2.4 & 0.7 & 2.5 & 1.9 & 2.83 & 0.31 & 4.70 \\
\hline WPSD132C & C. 136244 & Middie Waste & & 131.50 & 132.50 & 1.00 & 132.00 & 35.8 & 20.6 & 2.0 & $<0.1$ & 1.3 & 0.8 & 0.2 & 2.1 & 1.8 & 2.38 & 0.33 & 1.56 \\
\hline WPSD 132x & C. 136260 & & duplicate of previox & is semple & & & & 39.9 & 21.3 & 1.7 & $<0.1$ & 1.3 & 0.7 & 0.2 & 2.1 & 1.9 & 2.47 & 0.37 & 1.53 \\
\hline WPSD $137 \mathrm{C}$ & C.136277 & Niddle Waste & & 132.50 & 139.00 & 6.50 & 135.75 & 22.4 & 85.1 & 4.3 & 0.1 & 1.1 & 5.4 & 1.5 & 10.4 & 8.9 & 2.93 & 0.45 & 3.88 \\
\hline WPSO142C & C.136222 & Woddie Waste & & 139.00 & 144.00 & 5.00 & 141.50 & 23.3 & 45.9 & 3.2 & $<0.1$ & 1.1 & 5.4 & 1.5 & 8.0 & 6.6 & 2.53 & 0.36 & 4.10 \\
\hline WPSO147C & $\frac{c-13622}{C-136245}$ & Widile Waste & & $\frac{139.00}{144.00}$ & $\frac{14400}{150.00}$ & $\frac{3.00}{6.00}$ & $\frac{141.50}{147.00}$ & 21.3 & $\frac{45.9}{84.0}$ & $\frac{3.2}{3.8}$ & $<0.1$ & $\frac{1.1}{1.1}$ & $\frac{3.4}{3.2}$ & $\frac{1.5}{0.9}$ & 9.7 & $\frac{6.6}{8.8}$ & 2.53 & 0.40 & $\frac{3.10}{3.47}$ \\
\hline WPSO1S1C & $c-136252$ & Moddle Waste & dolominte & 150.00 & 151.00 & 1.00 & 150.50 & 4.3 & 10.0 & $<0.6$ & $<0.1$ & 0.2 & 41.2 & 11.2 & 11.8 & 0.6 & 0.34 & 0.07 & 0.72 \\
\hline & & -BREAX IN SECTION- & & & & & & & & & & & & & & & & & \\
\hline WPSO199C & C.136284 & Upper Ore Section, D Bed Ore & phosphonte & 199.00 & 200.00 & 1.00 & 199.50 & 13.7 & 4.1 & 1.4 & 0.1 & 0.9 & $0.8 \mathrm{r}>\mathrm{C}$ & 0.2 & 1. & 1.0 & 0.17 & 0.21 & 2.46 \\
\hline WPSO203C & C.136225 & Upper Waste & & 200.00 & 205 & 5.50 & & 21.5 & 51.1 & 3.4 & $<0$ & 3.2 & 5. & 1.6 & 5. & 4 & & 0. & 4.01 \\
\hline $0205 C$ & C.136253 & Upper Waste & wet mudstone & 204 & 205.50 & 1.00 & 205 & 29 & 56.9 & 2.9 & $<0.1$ & 4.3 & 0.3 & 0.1 & 5.0 & 4 & & & \\
\hline $0206 \mathrm{C}$ & C.136258 & Upper Waste & phosphonte & 205.50 & 206.25 & 0.75 & 205.88 & 14.5 & 23 & 0.9 & $<0$ & 0.5 & 0.9 & 0.2 & 1.4 & 1.2 & 0.4 & 0.17 & 1.32 \\
\hline WpS0206x & $c-136265$ & & duplacte of previou & ss sample & & & & 15.3 & 22.1 & $<0.6$ & $<0.1$ & 0.6 & 0.9 & 0.2 & 1.3 & 1.1 & 046 & 0.17 & 1.29 \\
\hline WPSO207C & C.136241 & Upper Waste & & 206.25 & 208.50 & 2.25 & 207.38 & 20.9 & 29.2 & 1.4 & $<0.1$ & 1.1 & 1.3 & 0.4 & 2.7 & 2.4 & 2.55 & 0.19 & 5.30 \\
\hline WPSO209C & C- 136280 & Upper Waste & phosphate nodule: & 208.50 & 209.00 & 0.50 & 208.75 & 10.8 & 29.1 & 1.1 & $<0.1$ & 1.0 & 0.5 & 0.1 & 2.7 & 2.6 & 0.94 & 0.27 & 3.38 \\
\hline WPSO210C & $c-136248$ & Uoper Waste & & 209.00 & 213.00 & 4.00 & 211.00 & 18.6 & 47.7 & 1.5 & $<0.1$ & 1.1 & 3.6 & 1.0 & 3.9 & 2.9 & 2.22 & 0.20 & 5.61 \\
\hline
\end{tabular}




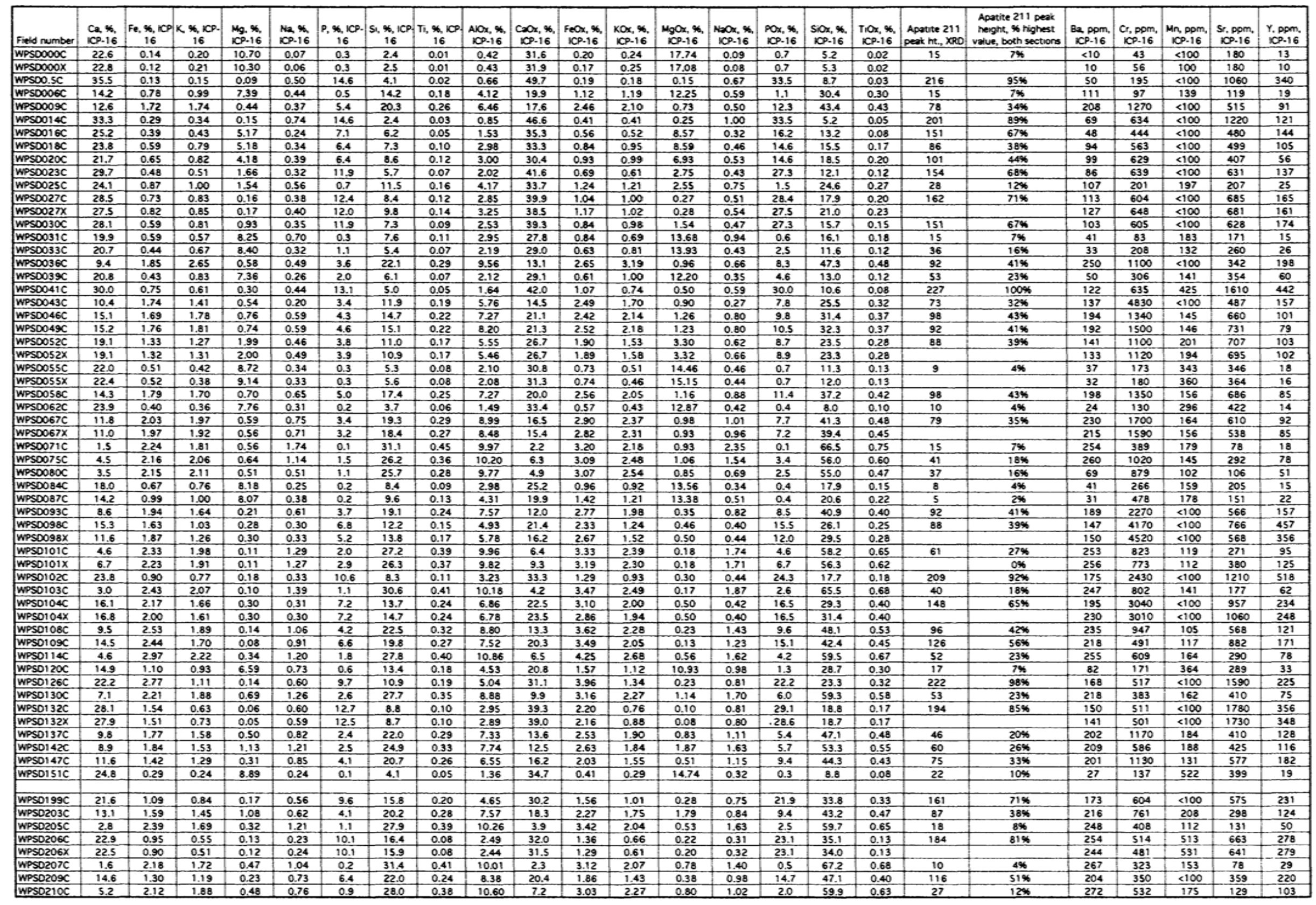

Table 2, Page 27 


\begin{tabular}{|c|c|c|c|c|c|c|c|c|c|c|c|c|c|c|c|c|c|c|c|c|c|c|c|c|}
\hline anumber & 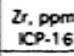 & & & & 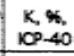 & 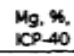 & & & $\begin{array}{l}l_{1,109} \\
40\end{array}$ & 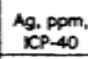 & 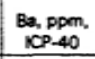 & 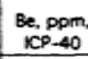 & 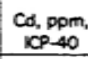 & $\begin{array}{c}c, p p m \\
c \in p-40 \\
C\end{array}$ & $\begin{array}{c}c a, p p m \\
c p \rightarrow 40\end{array}$ & $\begin{array}{c}a_{1, p o m} \\
c 00-40\end{array}$ & $\begin{array}{c}C_{u, p p m,} \\
\qquad \subset P-40\end{array}$ & 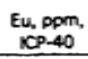 & $\begin{array}{c}\text { Ce ppom } \\
\text { cop } 40\end{array}$ & 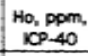 & 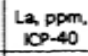 & $\begin{array}{l}\text { uspom, } \\
\text { icp.-40 }\end{array}$ & $\begin{array}{c}M_{n, p p m} \\
c_{C P A 40}\end{array}$ & 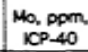 \\
\hline & 13 & 0.2 & $\frac{21.5}{21.5}$ & 0.1 & 0.1 & 11.0 & 0.0 & 0.3 & 0.0 & $<2$ & 8 & $<1$ & 150 & $<5$ & 42 & 35 & 14 & $<2$ & $<4$ & $<4$ & 8 & 2 & 94 & 10 \\
\hline$\frac{\text { WSPD0000x }}{\text { WPSDO0.5 }}$ & $\frac{17}{40}$ & $\frac{0.2}{0.3}$ & $\frac{16.9}{31.9}$ & $\frac{0.1}{0.1}$ & 0.1 & 8.4 & $\frac{0.0}{0.5}$ & $\frac{0.2}{1.47}$ & 0.0 & $<2$ & $\frac{7}{33}$ & $<1$ & 149 & $<5$ & $<2$ & 29 & 11 & $<2$ & $<4$ & $\frac{<4}{5}$ & $\frac{7}{260}$ & $\frac{<2}{5}$ & $\frac{76}{12}$ & \\
\hline WPSDocosc & 213 & $\begin{array}{l}2.3 \\
\end{array}$ & 14.0 & 0.8 & $\frac{0.6}{1.0}$ & $\frac{0.1}{7.6}$ & 0.5 & 0.5 & 0.1 & & 116 & $<1$ & $\frac{1250}{70}$ & $\frac{43}{23}$ & 3 & $\frac{101}{93}$ & 23 & $<2$ & 64 & $<4$ & $\frac{60}{17}$ & 9 & 133 & $\frac{10}{10}$ \\
\hline WPSDO0OSC & 237 & 3.5 & 12.6 & 1.7 & 1.8 & 0.4 & 0.4 & 5.4 & 0.2 & & 223 & $<1$ & 129 & 42 & & 671 & 167 & $<2$ & 14 & $<4$ & 71 & 30 & & \\
\hline 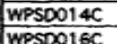 & 71 & 0.4 & 24.9 & 0.2 & 0.2 & $\frac{0.1}{5.2}$ & 0.6 & $\frac{12.3}{7.3}$ & 0.0 & & 60 & $<1$ & 87 & 12 & 4 & 382 & 88 & $<2$ & 4 & $<4$ & 85 & $\frac{7}{6}$ & $\frac{29}{71}$ & 21 \\
\hline 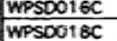 & $\begin{array}{l}63 \\
88\end{array}$ & $\frac{0.8}{1.6}$ & $\frac{22.1}{22.0}$ & 0.4 & $\begin{array}{l}0.5 \\
0.9\end{array}$ & 5.2 & $\frac{0.2}{0.4}$ & $\frac{7.1}{65}$ & 0.0 & & 43 & $<1$ & 99 & $\frac{22}{20}$ & & $\frac{204}{353}$ & $\frac{51}{63}$ & $<2$ & $<4$ & $<4$ & $\frac{121}{78}$ & & 71 & $\frac{15}{25}$ \\
\hline 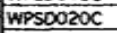 & & 1.4 & 16.9 & $\frac{0.6}{0.5}$ & 0.7 & $\frac{5.1}{3.5}$ & 0.4 & $\frac{6.5}{5.3}$ & 0.11 & & $\frac{91}{87}$ & $<1$ & $\begin{array}{l}92 \\
96\end{array}$ & $\frac{20}{14}$ & $\frac{4}{2}$ & $\frac{325}{346}$ & 53 & $\frac{<2}{<2}$ & $\frac{4}{<4}$ & $\frac{14}{<4}$ & 37 & $\frac{12}{9}$ & $\frac{82}{63}$ & $\begin{array}{l}35 \\
40\end{array}$ \\
\hline & 88 & & 26.6 & 0.4 & 0.5 & 1.6 & 0.3 & 12.1 & 0.0 & 3 & 76 & 1 & 81 & 17 & & 354 & 68 & 62 & 5 & $<4$ & 98 & 10 & 28 & 32 \\
\hline WPSDS025c & 137 & 2.3 & 23.1 & 0.9 & 1.0 & 1.5 & 0.6 & 0.6 & 0.1 & 2 & 110 & $<1$ & 258 & 19 & & 42 & 29 & $<2$ & $<4$ & $<4$ & 21 & 8 & 194 & 5 \\
\hline 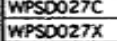 & $\frac{129}{154}$ & 1.6 & $\frac{25.0}{25.8}$ & $\frac{0.7}{0.5}$ & 0.8 & $\frac{0.1}{0.2}$ & 0.4 & $\frac{12.4}{120}$ & $\frac{0.1}{0.1}$ & $\frac{5}{5}$ & $\frac{112}{1122}$ & $<1$ & 45 & $\frac{33}{28}$ & 2 & 414 & $\frac{72}{75}$ & & 8 & $<4$ & 118 & 13 & 39 & 19 \\
\hline WPSDO0 30C & 99 & 1.4 & 27.9 & 0.5 & 0.8 & & & $\frac{2.0}{120}$ & $\frac{0.0}{0.0}$ & & $\frac{102}{103}$ & $<1$ & $\frac{40}{34}$ & $\frac{20}{29}$ & & 414 & 60 & & 5 & $\leqslant 4$ & 135 & $\frac{13}{12}$ & & \\
\hline insoso3ic & 86 & 1.7 & 19.4 & 0.6 & 0.6 & 8.5 & 0.7 & 0.3 & 0.1 & 2 & 44 & $<1$ & 22 & 15 & 4 & 18 & 19 & $<2$ & 5 & $<4$ & 14 & 2 & 189 & \\
\hline Wosposect & 51 & 1.3 & 20.6 & & 0.8 & 9.0 & 0.3 & 1.1 & 0.0 & & 32 & $<1$ & 22 & & & 244 & 30 & & $<4$ & $<4$ & 23 & $<2$ & 133 & 4 \\
\hline 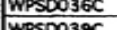 & 190 & 5.2 & 8.9 & 1.8 & 2.4 & 0.5 & 0.5 & 3.5 & 0.2 & 12 & 245 & $\leq 1$ & 35 & 61 & & 1040 & 91 & 4 & & $<4$ & 157 & & 55 & 16 \\
\hline wossoos $39 \mathrm{C}$ & $\frac{47}{85}$ & $\frac{1.0}{09}$ & $\frac{15.8}{27.4}$ & 0.4 & $\frac{0.7}{0.5}$ & 6.1 & $\frac{0.2}{0.4}$ & $\frac{1.7}{13.3}$ & 0.0 & $<2$ & 40 & $<1$ & 14 & 9 & $-\frac{2}{7}$ & $\frac{122}{356}$ & $\frac{29}{92}$ & $\frac{2}{7}$ & $<4$ & $<4$ & 45 & 2 & 1111 & 3 \\
\hline WPSD0043C & $\frac{53}{161}$ & & $\frac{2.04}{10.4}$ & & $\frac{1.5}{1.5}$ & $\frac{0.3}{0.5}$ & $\frac{0.4}{0.2}$ & $\frac{13.2}{39}$ & 0.0 & & $\frac{128}{148}$ & $<1$ & $\begin{array}{l}21 \\
988\end{array}$ & $\begin{array}{l}54 \\
37\end{array}$ & & $\frac{1560}{1530}$ & 93 & & $\frac{35}{15}$ & $\frac{7}{<4}$ & $\frac{329}{122}$ & & $\frac{410}{60}$ & 132 \\
\hline WPSDOAGC & 121 & $\begin{array}{l}3.1 \\
4.0 \\
\end{array}$ & 14.5 & $\frac{1.7}{1.7}$ & $\frac{1.3}{1.8}$ & 0.7 & 0.6 & 4.4 & 0.1 & $\frac{\infty}{5}$ & 203 & 2 & $\frac{300}{5}$ & 41 & & 695 & 110 & 2 & $\frac{13}{13}$ & $\leqslant 4$ & 89 & $\frac{20}{21}$ & $\frac{132}{132}$ & \\
\hline WPSD0049c & 117 & & & 1.7 & 1.9 & 0.6 & 0.6 & 4.3 & & & 180 & & 6 & 34 & & 1010 & 93 & & 14 & $<4$ & 67 & & 116 & $\frac{36}{36}$ \\
\hline WPSDOS2C & 110 & 3.1 & 17.3 & 1.3 & 1.4 & 1.7 & 0.5 & 3.8 & 0.1 & 4 & 134 & & 8 & 31 & & 406 & 73 & $\leq 2$ & 8 & $<4$ & 8 & & & 42 \\
\hline 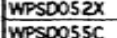 & 104 & 3.1 & $\frac{18.3}{220}$ & 1.3 & 1.4 & 2.0 & 0.5 & 4.0 & 0.1 & 3 & 140 & $\leq 1$ & 9 & 36 & & 328 & 76 & 2 & 6 & $<4$ & 90 & 16 & 192 & 39 \\
\hline WPSD0055x & 71 & $\frac{1 .}{10}$ & $\frac{2.20}{167}$ & 0.5 & 0.3 & $\frac{9.0}{72}$ & 0.4 & $\frac{0.3}{0.2}$ & $\frac{0.0}{0.0}$ & $\frac{24}{k^{2}}$ & $\frac{29}{23}$ & $<1$ & $<2$ & $\frac{8}{5}$ & & 69 & $\frac{20}{15}$ & & $\frac{14}{<4}$ & $<4$ & 17 & $<2$ & $\frac{342}{265}$ & 10 \\
\hline WPSDOSBC & 17 & 4.0 & 13.1 & 1.8 & 1.7 & $\frac{1.6}{0.7}$ & 0.6 & 4.7 & 0.1 & 3 & 188 & 2 & 3 & 41 & & 420 & 83 & 2 & 12 & $\leqslant 4$ & 73 & 21 & 138 & $\frac{8}{27}$ \\
\hline WPSDO062C & & 0.7 & 17.8 & 0.3 & 0.3 & 6.4 & 0.3 & 0.1 & 0.0 & $<2$ & 19 & & $<8$ & 6 & & 22 & 14 & & $\leqslant 4$ & $<4$ & $\ldots$ & $<2$ & 232 & \\
\hline WPSD0067c & 185 & 4.9 & 11.3 & 2.0 & 2.1 & 0.5 & 0.8 & 3.4 & 0.1 & 4 & 216 & 2 & 4 & 42 & & 996 & 90 & & 19 & $<4$ & 77 & 30 & 138 & 26 \\
\hline Wrs0067 $7 x$ & 176 & 4.7 & 10.4 & 2.0 & 2.0 & 0.5 & 0.7 & 3.1 & 0.2 & 3 & 219 & 2 & 3 & 42 & & 1350 & 85 & & 17 & $<4$ & 74 & 27 & 134 & 24 \\
\hline Wrosoo7sc & $\frac{410}{251}$ & $\frac{3.2}{5.5}$ & $\frac{1.5}{45}$ & $\frac{24}{22}$ & $\frac{1.3}{22}$ & 0.5 & 7.6 & $\frac{0.1}{1.5}$ & $\frac{0.2}{0.2}$ & -5 & $\frac{256}{250}$ & 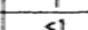 & $\frac{28}{7}$ & $\frac{49}{53}$ & 7 & $\frac{310}{233}$ & $\frac{24}{65}$ & & 17 & $x_{4}^{4}$ & & 19 & $\frac{155}{123}$ & -11 \\
\hline WPSDO0 BOC & 107 & 5.3 & 3.5 & 2.2 & 2.1 & 0.5 & 0.5 & $\frac{1.1}{1.1}$ & 0.2 & $<2$ & 72 & $<1$ & $<2$ & 40 & 12 & 454 & 36 & & 9 & $\leqslant 4$ & 7 & 3 & 71 & $\frac{37}{15}$ \\
\hline WPSDO084C & 44 & 1.3 & 12.9 & 0.5 & 0.6 & 6.6 & 0.2 & 0.1 & 0.0 & $<2$ & 30 & $<1$ & $<2$ & 7 & 3 & 36 & 16 & & $<4$ & 54 & & & 117 & 3 \\
\hline $087 \mathrm{c}$ & 49 & 2.0 & 11.0 & 0.8 & 0.8 & 6.6 & 0.3 & 0.2 & 0.1 & $<2$ & 24 & $<1$ & $<2$ & 12 & 4 & 69 & 20 & $<2$ & 6 & $<4$ & 25 & $<$ & 136 & 7 \\
\hline WPSDog3c & 145 & 4.2 & 8.6 & 2.0 & 1.7 & 0.2 & 0.6 & 3.9 & 0.1 & 16 & 201 & $<1$ & 16 & 50 & & 556 & 144 & & $\frac{13}{20}$ & $<4$ & 151 & 34 & 61 & $\frac{71}{153}$ \\
\hline $\mid$ & $\frac{107}{110}$ & $\frac{27}{32}$ & 14.7 & 1.6 & $\frac{1.1}{1.3}$ & 0.3 & 0.3 & $\frac{6.8}{5.3}$ & 0.1 & 16 & $\frac{146}{153}$ & 2 & $\frac{59}{58}$ & $\frac{51}{54}$ & & $\frac{1490}{1510}$ & $\frac{28 B}{300}$ & & $\frac{20}{20}$ & 6 & & & 34 & $\frac{153}{156}$ \\
\hline \begin{tabular}{|l|l|l|} 
WPSDO10 \\
\end{tabular} & 311 & 5.4 & 4.5 & 2.4 & 2.1 & 0.1 & 1.3 & 2.0 & 0.2 & 9 & 261 & 2 & 7 & 62 & & 281 & 62 & & 15 & $<4$ & 92 & 14 & 103 & 34 \\
\hline & 294 & 5.5 & 6.3 & 2.3 & 2.0 & 0.1 & 1.3 & 2.7 & 0.2 & 8 & 249 & 2 & 7 & 71 & 9 & 620 & 57 & 3 & 16 & $<4$ & 119 & 14 & 83 & 33 \\
\hline 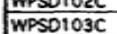 & $\frac{128}{338}$ & $\frac{1.8}{5.7}$ & $\frac{22.0}{30}$ & $\frac{0.9}{2.4}$ & $\frac{0.7}{22}$ & 0.2 & 0.3 & $\frac{10.5}{1.3}$ & & & $\frac{176}{245}$ & 4 & 53 & $\frac{64}{62}$ & 3 & $\frac{2460}{880}$ & $\frac{176}{56}$ & & $\frac{23}{15}$ & 9 & & $\frac{25}{12}$ & $\frac{27}{99}$ & 40 \\
\hline & 195 & 3.8 & 15.3 & $\frac{2.4}{22}$ & 1.7 & 0.0 & 1.4 & 72 & 0.1 & -8 & 200 & 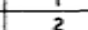 & 12 & 57 & 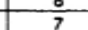 & $\frac{850}{1300}$ & $\frac{36}{162}$ & 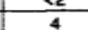 & $\frac{13}{28}$ & 5 & 214 & 44 & 69 & $\frac{26}{110}$ \\
\hline WPSO104x & 165 & 3.6 & 15.3 & 1.9 & 1.7 & 0.3 & 0.3 & 7.4 & 0.1 & 6 & 183 & 2 & 10 & 64 & 6 & 1060 & $\frac{102}{162}$ & 4 & 26 & 4 & 219 & 43 & 65 & 92 \\
\hline WPSO $108 C$ & 242 & 4.9 & 8.8 & 2.5 & 2.0 & 0.1 & 1.1 & 4.1 & 0.2 & 4 & 239 & 2 & 3 & 50 & 7 & 774 & 74 & & 17 & $<4$ & 100 & 23 & 87 & 30 \\
\hline WPSDD114C & 129 & $\frac{4.1}{5.4}$ & $\frac{14.5}{4.6}$ & $\frac{2.4}{30}$ & $\frac{1.7}{32}$ & 0.1 & 0.9 & 6.5 & 0.1 & $<2$ & $\frac{217}{266}$ & 2 & 42 & 56 & & & 5 & & 11 & 44 & 65 & 35 & 97 & $\frac{10}{19}$ \\
\hline WPSDI2OC & $\frac{541}{176}$ & $\frac{2.6}{26}$ & 1.6 & 1.1 & $\frac{2 .}{1.0}$ & $\begin{array}{l}.0 .3 \\
6.7\end{array}$ & 0.8 & 0.5 & 0.2 & $<2$ & $\frac{66}{86}$ & $<1$ & $<2$ & 27 & & $\frac{51}{51}$ & $\frac{35}{25}$ & $<2$ & 9 & $<4$ & 35 & 3 & 353 & \\
\hline WOSD & 86 & 27 & & 1.5 & & & 06 & 90 & & 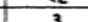 & ra & 2 & 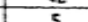 & 77 & & 35 & 66 & 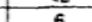 & 17 & 4 & 5 & $\because 1$ & 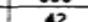 & 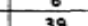 \\
\hline & 363 & 4.9 & 6.6 & 2.2 & 1.9 & 0.7 & 1.3 & 2.5 & 0.1 & $<2$ & 22 & 1 & $\leqslant 2$ & 52 & 8 & 305 & 30 & $<2$ & is & $\leqslant 4$ & 75 & 8 & $\frac{725}{125}$ & $\frac{39}{17}$ \\
\hline & 97 & 1.6 & 26.0 & 1.6 & 0.7 & 0.1 & 0.6 & 12.9 & 0.0 & $<2$ & 141 & 2 & 3 & 107 & & 329 & 38 & & 8 & 5 & 302 & 7 & 53 & 31 \\
\hline 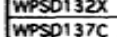 & $\frac{137}{272}$ & 7.6 & $\frac{26.3}{89}$ & $\frac{1.2}{1.8}$ & 0.7 & 0.1 & -0.6 & $\frac{12.5}{32}$ & 0.0 & $\frac{2}{6}$ & $-\frac{14}{21}$ & 2 & 49 & $\frac{107}{52}$ & & 399 & & & 8 & 7 & 295 & $\frac{7}{22}$ & 46 & 31 \\
\hline & $\frac{210}{310}$ & 4.1 & 8.1 & 1.8 & $\frac{1.6}{1.6}$ & 1.0 & 0.8 & 2.5 & $\frac{0.1}{02}$ & 6 & $\frac{12}{197}$ & $<$ & $\angle 9$ & $\frac{52}{59}$ & & & & & $\frac{14}{4}$ & 84 & 79 & $\frac{27}{10}$ & $\frac{101}{161}$ & $\frac{46}{32}$ \\
\hline & 265 & 3.6 & 11.4 & $\frac{1.4}{1.4}$ & 1.4 & 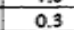 & 0.9 & 4.2 & 0.1 & 0 & & 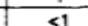 & 19 & 54 & & & 97 & & 12 & $\leqslant 4$ & 118 & 18 & 115 & 32 \\
\hline WPSDISIC & 36 & 0.6 & 18.1 & 0.2 & 0.2 & 7.0 & 0.2 & 0.1 & 0.0 & $<2$ & 17 & $<1$ & $<2$ & $<5$ & $<2$ & $\frac{350}{20}$ & 16 & $<2$ & $<4$ & $<4$ & 10 & $\frac{10}{<2}$ & 400 & 5 \\
\hline WFSO199C & 90 & 2.6 & 20.1 & 0.9 & 0.8 & 0.2 & 0.6 & 9.3 & 0.1 & 4 & 180 & $<1$ & 21 & 53 & & 355 & 47 & & 6 & $<4$ & 150 & 8 & 38 & \\
\hline & 233 & 4.2 & $\frac{12.0}{27}$ & $\frac{1.6}{2.6}$ & 1.6 & 10 & 0.6 & 4.2 & $0 ?$ & 6 & 210 & 11 & 80 & 43 & 6 & 702 & 63 & & 13 & 54 & & 27 & 191 & \\
\hline $50205 \mathrm{C}$ & 320 & 5.7 & $\frac{27}{212}$ & $\frac{2.4}{09}$ & 1.7 & 0.3 & 1.2. & 1.0 & 0.3 & $\begin{array}{c}3 \\
2 \\
2\end{array}$ & 237 & $<1$ & 37 & -47 & 11 & 413 & 38 & & 16 & $<4$ & 38 & 19 & 93 & \\
\hline $206 x$ & 96 & 1.4 & 22.4 & 0.4 & 0.5 & 0.1 & $\frac{0.4}{0.2}$ & 10.2 & 0.0 & $<2$ & 272 & $<1$ & 8 & 46 & 3 & 36 & 36 & & & 4 & $\frac{221}{230}$ & 7 & 4867 & \\
\hline Wpso207c & 379 & 5.4 & 1.6 & 2.2 & 1.8 & 0.5 & 1.5 & 0.2 & 0.2 & $<2$ & 290 & 2 & 5 & 25 & 8 & 123 & 30 & $<2$ & 12 & $<4$ & 20 & 18 & 141 & 17 \\
\hline WPSD2009C & $\begin{array}{r}100 \\
206\end{array}$ & \begin{tabular}{|l|}
3.4 \\
5.9
\end{tabular} & $\frac{12.6}{52}$ & 1.3 & 1.1 & 0.2 & 0.7 & \begin{tabular}{|l|}
6.1 \\
\end{tabular} & \begin{tabular}{|l}
0.1 \\
0.3 \\
\end{tabular} & \begin{tabular}{|l}
$<2$ \\
\end{tabular} & $\begin{array}{l}198 \\
277\end{array}$ & 1 & \begin{tabular}{|l} 
\\
\end{tabular} & \begin{tabular}{|l|}
89 \\
59
\end{tabular} & \begin{tabular}{|l|}
4 \\
\end{tabular} & 375 & 38 & 3 & 9 & $<4$ & $\begin{array}{l}161 \\
68\end{array}$ & 12 & 36 & 9 \\
\hline
\end{tabular}




\begin{tabular}{|c|c|c|c|c|c|c|c|c|c|c|c|c|}
\hline Freld number & $\begin{array}{l}\text { Nb, ppm, } \\
\text { COP } 40\end{array}$ & 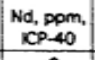 & $\begin{array}{c}\mathrm{N}, \mathrm{ppm}, \\
\mathrm{N} C \mathrm{CP}-40 \\
\end{array}$ & $\begin{array}{l}\text { Pb, ppm, } \\
\text { ICP. } 40 \\
\end{array}$ & $\begin{array}{c}\text { Sc, ppm, } \\
\text { icp } 40\end{array}$ & $\begin{array}{l}\text { Th, ppm. } \\
\text { icp }-40\end{array}$ & $\begin{array}{l}\text { u, ppm, } \\
1 C^{2}-40\end{array}$ & $\begin{array}{c}v_{1} \text { ppom, } \\
\text { iCP } 40\end{array}$ & 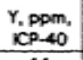 & 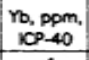 & $\begin{array}{c}2 n_{1}, \mathrm{ppm}, \\
\mathrm{CPP}-40\end{array}$ & $\begin{array}{l}\text { eli, unscialed, pom, } \\
\text { chamel interval avg. }\end{array}$ \\
\hline WPSDO000 C & $<4$ & $<9$ & 45 & $<4$ & $<<2$ & $<6$ & $<100$ & 62 & 11 & $<1$ & 450 & 125 \\
\hline WPSD000x & $\leq 4$ & $<9$ & 44 & $<4$ & $<2$ & $<6$ & $<100$ & 49 & 9 & $<1$ & 436 & \\
\hline WPSDo.sC & $<4$ & 163 & 233 & 12 & $<2$ & 6 & $<100$ & 130 & 351 & 13 & 2640 & 87 \\
\hline WPSD0006C & $<4$ & $<9$ & 172 & 4 & 3 & $<6$ & $<100$ & 402 & 17 & 2 & 1300 & 38 \\
\hline WPSD0009C & $<4$ & 47 & 341 & 18 & 7 & 11 & $<100$ & 3190 & 96 & 7 & 2290 & 109 \\
\hline WPSDO014C & $<4$ & 32 & 106 & 13 & $<2$ & $<6$ & 198 & 1360 & 117 & 5 & 794 & 192 \\
\hline WPSDO16C & $<4$ & 46 & 141 & 4 & 3 & $<6$ & $<100$ & 195 & 149 & 6 & 1050 & 63 \\
\hline WPSDO18C & $<4$ & 30 & 193 & 8 & 3 & $<6$ & $<100$ & 533 & 109 & 5 & 1570 & 85 \\
\hline WPSO0Z20C & $<4$ & 16 & 181 & 8 & 2 & 7 & $<100$ & 780 & 53 & 3 & 1610 & 150 \\
\hline WPSDD023C & $<4$ & 37 & 129 & 11 & $<2$ & $<6$ & $<100$ & 1010 & 142 & 6 & 1240 & 152 \\
\hline WPSDO25C & $\leq 4$ & 11 & 167 & 9 & 4 & $<6$ & $<100$ & 480 & 26 & 2 & 1140 & 114 \\
\hline WiPSD0027C & $<4$ & 53 & 150 & 13 & $<2$ & 8 & 118 & 1680 & 172 & 8 & 918 & 125 \\
\hline WPSD0027x & $<4$ & 49 & 165 & 12 & $\leq 2$ & $<6$ & 109 & 1810 & 167 & 8 & 1010 & \\
\hline WPSD0030C & $<4$ & 56 & 126 & 9 & $\leqslant 2$ & $<6$ & $\leq 100$ & 788 & 187 & 7 & 712 & 92 \\
\hline WPSDDO31C & $<4$ & $<9$ & 105 & $<4$ & 2 & $<6$ & $<100$ & 102 & 16 & 1 & 594 & 42 \\
\hline WPSO033C & $<4$ & $<9$ & 55 & $\leq 4$ & $<2$ & $<6$ & $<100$ & 143 & 27 & 2 & 288 & 29 \\
\hline WPSO036C & 4 & 84 & 267 & 15 & 11 & 8 & $<100$ & 478 & 205 & 9 & 1110 & 48 \\
\hline WPSDO39C & $<4$ & 19 & 62 & $\leq 4$ & 2 & $<6$ & $<100$ & 146 & 58 & 3 & 250 & 48 \\
\hline WPSDOA1C & $<4$ & 174 & 275 & 13 & 5 & 7 & $<100$ & 439 & 469 & 20 & 819 & 79 \\
\hline WPSDO43C & $<4$ & 93. & 1400 & 44 & 11 & 14 & $<100$ & 6050 & 166 & 13 & 8220 & 68 \\
\hline WPSDOA6C & $<4$ & 50 & 383 & 9 & 10 & 8 & $<100$ & 145 & 106 & 6 & 1380 & 40 \\
\hline WPSDOASC & $<4$ & 47 & 298 & 12 & 8 & 8 & $<100$ & 140 & 78 & 4 & 1250 & 37 \\
\hline WPSDDoszC & $<4$ & 47 & 247 & 5 & 7 & $<6$ & $<100$ & 153 & 108 & 5 & 960 & 35 \\
\hline WPSD0s2X & $<4$ & 47 & 249 & 4 & 8 & $<6$ & $<100$ & 163 & 108 & 6 & 938 & \\
\hline WPSDOS5C & $<4$ & 9 & 37 & $\leq 4$ & $<2$ & $<6$ & $<100$ & 40 & 18 & $<1$ & 166 & 21 \\
\hline WPSD0055x & $<4$ & $<9$ & 27 & $<4$ & $<2$ & $<6$ & $<100$ & 32 & 14 & $\leq 1$ & 115 & \\
\hline WPSDO5BC & $<4$ & 49 & 306 & 7 & 9 & $<6$ & $<100$ & 114 & 88 & 5 & 1260 & 29 \\
\hline WPSDO062C & $<4$ & $<9$ & 19 & $<4$ & $<2$ & $<6$ & $<100$ & 27 & 12 & $<1$ & 110 & 26 \\
\hline WPSD067C & $<4$ & 54 & 316 & 12 & 11 & 8 & $<100$ & 145 & 94 & 5 & 1230 & 28 \\
\hline WPSD067x & $\angle 4$ & 51 & 312 & 14 & 11 & 7 & $<100$ & 141 & 90 & 5 & 1180 & \\
\hline WPSDO71C & 9 & 16 & 110 & 19 & 7 & 8 & $<100$ & 131 & 10 & 2 & 330 & 22 \\
\hline WPSD0075C & 5 & 46 & 304 & 18 & 11 & 11 & $<100$ & 249 & 79 & 5 & 1230 & 27 \\
\hline WPSDOBOC & 5 & 42 & 1490 & 15 & 14 & $<6$ & $<100$ & 339 & 53 & 4 & 14530 & 18 \\
\hline WPSDOBAC & $<4$ & $<9$ & 38 & $\leq 4$ & 3 & $<6$ & $<100$ & 40 & 13 & $<1$ & 184 & 17 \\
\hline WPSD087C & $<4$ & 11 & 223 & 4 & 5 & $<6$ & $<100$ & 97 & 20 & 1 & 2370 & 28 \\
\hline WPSD0 $933 \mathrm{C}$ & $<4$ & 83 & 617 & 17 & 12 & 8 & $<100$ & 206 & 170 & 8 & 3200 & 52 \\
\hline WPSDO098C & $<4$ & 173 & 991 & 17 & 11 & 11 & $<100$ & 312 & 471 & 18 & 4070 & 100 \\
\hline WPSD0098X & $<4$ & 148 & 1050 & 16 & 11 & 8 & $<100$ & 324 & 383 & 14 & 4110 & \\
\hline WPSO101C & $<4$ & 53 & 226 & 18 & 10 & 9 & $<100$ & 136 & 101 & 6 & 1160 & 48 \\
\hline WPSO101X & $<4$ & 69 & 205 & 17 & 9 & 10 & $<100$ & 115 & 129 & 6 & 987 & \\
\hline WPSD $102 C$ & $<4$ & 216 & 492 & 11 & 9 & 10 & $<100$ & 149 & 541 & 20 & 2470 & 38 \\
\hline WPSO103C & 8 & 42 & 172 & 19 & 9 & $\frac{10}{7}$ & $<100$ & 102 & 65 & 4 & 922 & 32 \\
\hline WPSO104C & $<4$ & 108 & 562 & 12 & 10 & 7 & $<100$ & 202 & 250 & 10 & 2170 & 45 \\
\hline WPSD104X & $<4$ & 112 & 538 & 9 & 11 & 9 & $<100$ & 192 & 257 & 11 & 2140 & \\
\hline WPSD 108C & 4 & 64 & 209 & 12 & 9 & 11 & $<100$ & 130 & 126 & 6 & 702 & 52 \\
\hline WPSDI09C & $<4$ & 115 & 116 & 13 & 9 & 6 & $<100$ & 93 & 180 & 8 & 541 & 53 \\
\hline WPSO1 14C & 6 & 50 & 168 & 17 & 11 & 8 & $<100$ & 108 & 83 & 5 & 563 & 31 \\
\hline WPSDI $20 \mathrm{C}$ & $<4$ & 14 & 43 & $<4$ & 4 & $<6$ & $<100$ & 45 & 34 & 2 & 136 & 21 \\
\hline WPSD 126C & $<4$ & 132 & 166 & 9 & $<2$ & $<6$ & $<100$ & 92 & 238 & 11 & 648 & 50 \\
\hline WPSDI $30 \mathrm{C}$ & $<4$ & 41 & 90 & 13 & 9 & 9 & $<100$ & 79 & 77 & 4 & 290 & 29 \\
\hline WPSD $132 C$ & $<4$ & 192 & 93 & 13 & 7 & 12 & $<100$ & 64 & 374 & 15 & 425 & 42 \\
\hline WPSD132X & $<4$ & 187 & 86 & 10 & $<2$ & 8 & $<100$ & 60 & 367 & 14 & 395 & \\
\hline WPSO1 $37 C$ & $<4$ & 54 & 318 & 11 & 8 & 8 & $<100$ & 233 & 130 & 7 & 1100 & 38 \\
\hline WPSO142C & 5 & 50 & 159 & 10 & $\frac{8}{7}$ & 10 & $<100$ & 146 & 118 & 7 & 428 & $\frac{28}{23}$ \\
\hline WPSO147c & $<4$ & 70 & 291 & 10 & 7 & 8 & $<100$ & 189 & 191 & 9 & 1040 & 32 \\
\hline WPSDISIC & $<4$ & $<9$ & 19 & $<4$ & $<2$ & $<6$ & $<100$ & 29 & 15 & $<1$ & 66 & 33 \\
\hline WPSD199C & $<4$ & 81 & 90 & 10 & $<2$ & $<6$ & $<100$ & 279 & 246 & 11 & 600 & 134 \\
\hline WPSO203C & $<4$ & 49 & 186 & 14 & 8 & 11 & $<100$ & 269 & 128 & 7 & 1270 & 120 \\
\hline WPSOZOSC & 8 & 32 & 231 & 23 & 10 & 10 & $<100$ & 339 & 50 & 4 & 1640 & 96 \\
\hline WPSO2006C & $<4$ & 151 & 90 & 16 & 3 & 8 & $<100$ & 126 & 293 & 10 & 423 & \\
\hline WPSD206X & $<4$ & 160 & 88 & 10 & $<2$ & $<6$ & $<100$ & 118 & 302 & 10 & 418 & 112 \\
\hline WPSO207C & 7 & 20 & 113 & 17 & 9 & 7 & $<100$ & 122 & 29 & 3 & 333 & 71 \\
\hline WPSO209C & $<4$ & 102 & 126 & & 6 & 11 & $<100$ & 93 & 230 & 10 & 553 & \\
\hline WPSD210C & 9 & 58 & 206 & 15 & 10 & 10 & $<100$ & 118 & 108 & 6 & 466 & \\
\hline
\end{tabular}




\begin{tabular}{|c|c|c|c|c|c|c|c|c|c|c|c|c|c|c|c|c|c|}
\hline $\begin{array}{l}\text { ACCURACY-Standard } \\
\text { Reference SARL }\end{array}$ & Lab No. & $\begin{array}{l}\text { As, ppom, } \\
\text { Hyd. }\end{array}$ & $\begin{array}{l}\text { Se, ppm, } \\
\text { Hyd. }\end{array}$ & $\begin{array}{c}\text { So, ppm, } \\
\text { Hyd. }\end{array}$ & $\begin{array}{c}\text { Te, ppm, } \\
\text { FAA }\end{array}$ & $\begin{array}{c}\mathrm{T}, \mathrm{ppm}, \\
\text { FAA }\end{array}$ & $\begin{array}{c}\text { C, } \boldsymbol{\Phi}_{\text {, }} \\
\text { Combustion }\end{array}$ & $\begin{array}{c}\mathrm{CO2}, \% \\
\text { Acidification }\end{array}$ & $\begin{array}{c}\text { Carbonate C, } \% \text {, } \\
\text { Acidification }\end{array}$ & $\begin{array}{c}\text { Organic C, } \%, \\
\text { difference }\end{array}$ & $\begin{array}{c}\mathbf{S ,} \boldsymbol{*}_{\boldsymbol{r}} \\
\text { Combustion }\end{array}$ & $\begin{array}{l}\mathrm{Hg}, \mathrm{ppm}, \\
\text { CVAA }\end{array}$ & \begin{tabular}{|c|} 
A, \%, ICP- \\
16 \\
\end{tabular} & $\begin{array}{c}\text { Ca, \%, ICP. } \\
16\end{array}$ & $\begin{array}{c}\mathrm{Fe}, \%, \mathrm{iCP}- \\
16\end{array}$ & $\begin{array}{c}K, \$_{1}, \mathrm{ICP}- \\
16\end{array}$ & $\begin{array}{c}\text { Mg. } \%, \text { ICP- } \\
16 \\
\end{array}$ \\
\hline anayzed as & c-136289 & 16.1 & 0.9 & 4.8 & 0.7 & 1.1 & 0.41 & 0.11 & \begin{tabular}{|c|}
1.1 \\
\end{tabular} & 0.99 & 0.08 & 0.18 & \begin{tabular}{|l|}
5.70 \\
\end{tabular} & 1.10 & 2.54 & 2.86 & 0.50 \\
\hline analyzed as & C-136291 & 16.1 & 0.8 & 5.2 & 0.7 & 1.2 & 0.4 & 0.11 & 1.1 & 0.99 & 0.07 & 0.17 & 5.82 & 1.09 & 2.61 & 2.96 & 0.52 \\
\hline analyzed as & $c-136293$ & 17.8 & 0.8 & 4.6 & 0.7 & 1.2 & 0.4 & 0.11 & 1.1 & 0.99 & 0.08 & 0.19 & 5.83 & 1.05 & 2.56 & 2.95 & 0.52 \\
\hline Average & & 16.7 & 0.8 & 4.9 & 0.7 & 1.2 & 0.4 & 0.11 & 1.1 & 0.99 & 0.08 & 0.18 & 5.78 & 1.08 & 2.57 & 2.92 & 0.51 \\
\hline Accepted Value & & 16.5 & 0.9 & 5.1 & 0.6 & 1.4 & 0.40 & 0.11 & 0.97 & 0.86 & 0.07 & 0.16 & 5.79 & 1.06 & 2.67 & 2.98 & 0.55 \\
\hline Ret. Std. Diff. & & $1 \%$ & $-7 \%$ & $-5 \%$ & $17 \%$ & $-17 \%$ & $1 \%$ & $0 x$ & $13 \%$ & $15 \%$ & $10 \%$ & $16 \%$ & Ox & $2 \%$ & $-4 \%$ & $-2 \%$ & $-7 \%$ \\
\hline \multicolumn{18}{|l|}{$\begin{array}{l}\text { Standard Reference } \\
\text { SARM }\end{array}$} \\
\hline \begin{tabular}{|l|} 
analyzed as \\
\end{tabular} & $C-136290$ & 37.2 & 0.3 & 6.2 & 1.0 & 2.4 & 0.09 & 0.02 & 0.3 & 0.28 & 0.12 & 0.12 & 5.98 & 0.58 & 3.09 & 2.88 & 0.46 \\
\hline \begin{tabular}{|l} 
analyzed as \\
\end{tabular} & C-136292 & 37.9 & 0.3 & 6.2 & 1.1 & 2.4 & 0.09 & 0.02 & 0.28 & 0.26 & 0.12 & 0.11 & 5.87 & 0.56 & 2.93 & 2.84 & 0.45 \\
\hline \begin{tabular}{|l} 
analyzed as \\
\end{tabular} & $C-136294$ & 40.8 & 0.3 & 6.1 & 1.0 & 2.6 & 0.08 & 0.02 & 0.30 & 0.28 & 0.11 & 0.12 & 6.1 & 0.58 & 3.12 & 2.95 & 0.47 \\
\hline Average & & 38.6 & 0.3 & 6.2 & 1.0 & 2.5 & 0.09 & 0.02 & 0.29 & 0.27 & 0.12 & 0.12 & 5.98 & 0.57 & 3.05 & 2.89 & 0.46 \\
\hline Accepted Value & & 37 & 0.33 & 5.6 & 0.68 & 2.8 & 0.07 & 0.02 & 0.30 & 0.28 & 0.13 & 0.117 & 6.09 & 0.58 & 3.22 & 2.92 & 0.50 \\
\hline Ret. Std. Diff. & & $4 \%$ & $-9 \%$ & $10 \%$ & $52 \%$ & $-12 \%$ & $24 \%$ & $0 \%$ & $-2 \%$ & $-2 \%$ & $-10 \%$ & $0 \%$ & $-2 \%$ & -18 & $-5 \%$ & $-1 \%$ & $-8 \%$ \\
\hline \multicolumn{18}{|l|}{$\begin{array}{l}\text { PBV-1 Bone Valley } \\
\text { Phosphorite, split } 16\end{array}$} \\
\hline analyzed as & C-122917 & 12.6 & 2.5 & 1.8 & 0.2 & 1.7 & 3.84 & 1.05 & 1.45 & 0.40 & 0.55 & 0.09 & 0.54 & 34.8 & 0.90 & 0.12 & 0.24 \\
\hline anatyzed as & C-123874 & 10.5 & 2.6 & 1.6 & 0.1 & 2.1 & 3.85 & 1.05 & 1.46 & 0.41 & 0.58 & 0.09 & 0.52 & 33.6 & 0.85 & 0.13 & 0.23 \\
\hline Average & & 11.6 & 2.55 & 1.7 & 0.15 & 1.9 & 3.845 & 1.05 & 1.455 & 0.405 & 0.57 & 0.09 & 0.53 & 34.2 & 0.88 & 0.13 & 0.24 \\
\hline Rel. Std. Dev. & & $13 \%$ & $3 \%$ & $8 \%$ & $47 \%$ & $15 \%$ & $0.2 \%$ & $0.0 \%$ & $0.5 \%$ & $2 \%$ & $4 x$ & $0 \%$ & $3 \%$ & $2 \%$ & $4 \%$ & $6 \%$ & $3 \%$ \\
\hline \multicolumn{18}{|l|}{$\begin{array}{l}\text { Project Standards, no } \\
\text { replication }\end{array}$} \\
\hline POW-1 & C-136227 & 10.9 & 54.3 & 1.5 & $<0.1$ & 1.9 & 0.15 & 0.04 & 3.03 & 2.99 & 1.29 & 0.22 & 2.22 & 3.02 & 1 & 0.56 & 0.2 \\
\hline POW-2 & C-136247 & 31.9 & 134 & 7 & $<0.1$ & 2.4 & 4.46 & 1.22 & 7.52 & 6.3 & 0.86 & 0.49 & 3.48 & 14.8 & 1.45 & 1.31 & 1.09 \\
\hline$P O A-1$ & $c-136229$ & 19.1 & 43.1 & 1.8 & $<0.1$ & 1.5 & 2.49 & 0.68 & 3.7 & 3.02 & 2.55 & 0.22 & 5.83 & 4.13 & 2.39 & 1.97 & 1.07 \\
\hline \multicolumn{18}{|l|}{$\begin{array}{l}\text { PRECISION- duplicates } \\
\text { analyses } \\
\end{array}$} \\
\hline \multicolumn{18}{|l|}{ Section C } \\
\hline Number & & 6 & 6 & 6 & 2 & 6 & 6 & 6 & 6 & 6 & 6 & 6 & 6 & 6 & 6 & 6 & 6 \\
\hline Avg. Std. Diff. & & $9 \%$ & $6 \%$ & $11 \%$ & $20 \%$ & $17 \%$ & $11 \%$ & $4 \%$ & $3 \%$ & $5 \%$ & $34 \%$ & $3 \%$ & $8 \%$ & $9 \%$ & $7 \%$ & $8 \%$ & $8 \%$ \\
\hline Avg. Std. Dev. & & $6 \%$ & $4 \%$ & $8 \%$ & $14 \%$ & $12 \%$ & $8 \%$ & $3 \%$ & $2 \%$ & $3 \%$ & $24 \%$ & $2 \%$ & $5 \%$ & $7 \%$ & $5 \%$ & $6 \%$ & $6 \%$ \\
\hline \multicolumn{18}{|l|}{ Section D } \\
\hline Number & & 10 & 10 & 8 & 1 & 10 & 10 & 10 & 10 & 10 & 9 & 9 & 10 & 10 & 10 & 10 & 10 \\
\hline Avg. Std. Diff. & & $8 \%$ & $14 \%$ & $13 \%$ & $67 \%$ & $23 \%$ & $7 \%$ & $5 \%$ & $3 \%$ & $12 \%$ & $5 \%$ & $10 \%$ & $5 \%$ & $9 \%$ & $7 \%$ & $7 \%$ & $5 \%$ \\
\hline Avg. Std. Dev. & & $6 \%$ & $10 \%$ & $9 \%$ & $47 \%$ & $16 \%$ & $5 \%$ & $4 \%$ & $2 \%$ & $8 \%$ & $4 \%$ & $7 \%$ & $3 \%$ & $6 \%$ & $5 \%$ & $5 \%$ & $4 \%$ \\
\hline
\end{tabular}




\begin{tabular}{|c|c|c|c|c|c|c|c|c|c|c|c|c|c|c|c|c|c|c|c|}
\hline $\begin{array}{l}\text { ACCURACY-Standard } \\
\text { Reference SARL }\end{array}$ & \begin{tabular}{|c|}
$\mathrm{Na}, \%$, ICP- \\
16
\end{tabular} & $\begin{array}{l}P, \%, 1 C P \\
16\end{array}$ & $\begin{array}{c}\text { Si, } \%, \text { ICP- } \\
16\end{array}$ & $\begin{array}{c}T, 9,1, P \text { - } \\
16\end{array}$ & $\begin{array}{l}\text { Ba, ppm, } \\
\text { ICP-16 }\end{array}$ & $\begin{array}{l}\mathrm{Cr}, \mathrm{ppm}, \\
\mathrm{ICP}-16\end{array}$ & $\begin{array}{l}\text { Mn, ppm, } \\
\text { ICP-16 }\end{array}$ & $\begin{array}{l}\text { Nb, ppm, } \\
\text { ICP-16 }\end{array}$ & $\begin{array}{l}\text { Sr, ppm, } \\
\text { ICP-16 }\end{array}$ & $\begin{array}{c}\text { Y, Ppm, KCP } \\
16\end{array}$ & $\begin{array}{c}2 r, \mathrm{pPm}, \mathrm{ICP} \\
16\end{array}$ & $\begin{array}{c}\text { AN, } \\
40 \text { ICP- } \\
40\end{array}$ & $\begin{array}{c}\text { Ca, ppm, } \\
\text { ICP } 40\end{array}$ & $\begin{array}{c}\mathrm{Fe}, \boldsymbol{N}_{4}, \mathrm{ICP}-\mathrm{s} \\
40\end{array}$ & $\begin{array}{c}K, 9,1 C P- \\
40\end{array}$ & $\begin{array}{c}\text { Mg. \%, ICP. } \\
40\end{array}$ & $\begin{array}{c}\mathrm{Na}, \%, \mathrm{ICP} \\
40\end{array}$ & $\begin{array}{c}P, \%, I C P- \\
40\end{array}$ & $\begin{array}{c}\mathrm{Ti}, \%, \mathrm{ICP}-\mathrm{c} \\
40\end{array}$ \\
\hline anajyzod as & \begin{tabular}{l|l}
1.47 \\
\end{tabular} & 0.08 & 31.3 & 0.29 & 827 & 101 & 2010 & 38 & 142 & 53 & 358 & 6.06 & 1.10 & \begin{tabular}{|l|}
2.58 \\
\end{tabular} & 2.93 & \begin{tabular}{|l|}
0.52 \\
\end{tabular} & \begin{tabular}{|l|}
1.55 \\
\end{tabular} & \begin{tabular}{|l|l|}
0.08 \\
\end{tabular} & \begin{tabular}{|l|} 
\\
\end{tabular} \\
\hline anabyzod as & 1.50 & 0.07 & 32.9 & 0.31 & 871 & 112 & 2120 & 31 & 151 & 53 & 358 & 5.93 & 1.09 & 2.60 & 2.91 & 0.51 & 1.47 & 0.07 & 0.29 \\
\hline analyzed as & 1.51 & 0.07 & 32.6 & 0.30 & 868 & 110 & 2110 & 39 & 150 & 53 & 372 & 5.97 & 1.05 & 2.53 & 2.89 & 0.50 & 1.52 & 0.07 & 0.29 \\
\hline Average & 1.49 & 0.07 & 32.3 & 0.30 & 855 & 108 & 2080 & 36 & 148 & 53 & 363 & 5.98 & 1.08 & 2.57 & 2.91 & 0.51 & 1.51 & 0.07 & 0.29 \\
\hline Accepted Value & 1.53 & 0.09 & 33.6 & 0.25 & 879 & 110 & 2094 & 35 & 158 & 44 & 408 & 5.79 & 1.06 & 2.67 & 2.98 & 0.55 & 1.53 & 0.09 & 0.25 \\
\hline Rel. Std. Diff. & $-2 \%$ & $-19 \%$ & $-4 \%$ & $20 \%$ & $-3 \%$ & $-2 \%$ & $-1 \times$ & $3 \%$ & $-7 \%$ & $20 \%$ & $-11 \%$ & $3 \%$ & $2 \%$ & $-4 \%$ & $-2 \%$ & $-7 \%$ & $-1 \%$ & $-18 \%$ & $16 \%$ \\
\hline \multicolumn{20}{|l|}{$\begin{array}{l}\text { Standard Reference } \\
\text { SARM }\end{array}$} \\
\hline anabzed as & 1.15 & 0.07 & 32.5 & 0.35 & 762 & 98 & 5310 & 42 & 150 & 34 & 348 & 6.06 & 0.58 & 3.04 & 2.88 & 0.44 & 1.09 & 0.07 & 0.34 \\
\hline analyzed as & 1.10 & 0.06 & 31.2 & 0.34 & 735 & 101 & 5040 & 25 & 145 & 35 & 316 & 6.04 & 0.56 & 2.96 & 2.88 & 0.45 & 1.10 & 0.07 & 0.34 \\
\hline analyzed as & 1.16 & 0.07 & 32.8 & 0.37 & 776 & 116 & 5360 & 36 & 153 & 37 & 361 & 6.18 & 0.58 & 3.02 & 2.94 & 0.45 & 1.11 & 0.07 & 0.34 \\
\hline Average & 1.14 & 0.07 & 32.2 & 0.35 & 758 & 105 & 5237 & 34 & 149 & 35 & 342 & 6.09 & 0.58 & 3.01 & 2.90 & 0.45 & 1.10 & 0.07 & 0.34 \\
\hline Accepted Value & 1.19 & 0.08 & 33.5 & 0.35 & 764 & 101 & 5200 & 31 & 156 & 33 & 370 & 6.09 & 0.58 & 3.22 & 2.92 & 0.50 & 1.19 & 0.08 & 0.35 \\
\hline Rel. Std. Diff. & $4 x$ & $-17 \%$ & $-4 \%$ & $1 \%$ & $-1 \%$ & $4 \mathbf{x}$ & $1 \%$ & $11 \%$ & $-4 x$ & $7 \%$ & $-8 \%$ & $0 \%$ & $-1 \%$ & $-7 \%$ & $-1 x$ & $-11 \%$ & $-7 \%$ & $-17 \%$ & $-3 x$ \\
\hline \multicolumn{20}{|l|}{$\begin{array}{l}\text { Pav-1 Bone Valley } \\
\text { Phosphorite, split } 16\end{array}$} \\
\hline anejyzed as & 0.41 & 14.7 & 1.84 & 0.1 & 87 & 91 & 355 & $<10$ & 1160 & 163 & 226 & 0.56 & 31.3 & 0.59 & 0.15 & 0.25 & 0.54 & 14 & 0.048 \\
\hline anelyzed as & 0.46 & 13.9 & 1.81 & 0.1 & 86 & 51 & 313 & 17 & 1270 & 175 & 109 & 0.58 & 31.0 & 0.87 & 0.17 & 0.24 & 0.54 & 14 & 0.050 \\
\hline \begin{tabular}{|l|} 
Average \\
\end{tabular} & 0.44 & 14.3 & 1.83 & 0.1 & 86.5 & 71 & 334 & 17 & 1215 & 169 & 168 & 0.57 & 31.2 & 0.73 & 0.16 & 0.24 & 0.54 & 14 & 0.049 \\
\hline Rel. Std Dev. & $8 \%$ & $4 x$ & $1 \%$ & $0 \%$ & $1 \%$ & $40 \%$ & $9 \%$ & & $6 \%$ & $5 \%$ & $49 \%$ & $2 \%$ & $1 \%$ & $27 \%$ & $9 \%$ & $1 \%$ & $1 \%$ & $0 \%$ & $3 \%$ \\
\hline \multicolumn{20}{|l|}{$\begin{array}{l}\text { Project Standards, no } \\
\text { replication }\end{array}$} \\
\hline pow-1 & 0.07 & 1.28 & 38 & 0.11 & 172 & 607 & $<100$ & $<10$ & 138 & 123 & 82 & 1.99 & 3.0 & 0.99 & 0.57 & 0.182 & 0.074 & 1.292 & 0.085 \\
\hline pow-2 & 0.56 & 5.62 & 15.8 & 0.23 & 246 & 1270 & $<100$ & $<10$ & 579 & 166 & 182 & 3.54 & 14.5 & 1.46 & 1.41 & 1.054 & 0.58 & 5.764 & 0.116 \\
\hline POH-1 & 0.56 & 1.13 & 26.8 & 0.39 & 255 & 501 & 229 & $<10$ & 130 & 91 & 279 & 6.08 & 4.1 & 2.45 & 2.13 & 1.086 & 0.584 & 1.201 & 0.268 \\
\hline \multicolumn{20}{|l|}{$\begin{array}{l}\text { PRECCSION- duplicates } \\
\text { analyses }\end{array}$} \\
\hline \multicolumn{20}{|l|}{ Section C } \\
\hline Number & 6 & 6 & 6 & 6 & 6 & 6 & 1 & 1 & 6 & 6 & 6 & 6 & 6 & 6 & 6 & 6 & 6 & 6 & 6 \\
\hline Avg. Std. Diff. & $7 \%$ & $10 \%$ & $7 \%$ & $7 \%$ & $16 \times$ & $10 \%$ & $6 \%$ & $24 \%$ & $8 \%$ & $7 \times$ & $8 \%$ & $5 \%$ & $6 \%$ & $15 \%$ & $6 \%$ & $5 \%$ & $6 \%$ & $7 \%$ & $25 \%$ \\
\hline Avo. Std. Dev. & $5 \%$ & $7 \%$ & $5 \%$ & $5 \%$ & $11 \%$ & $7 \%$ & $5 \%$ & $17 \%$ & $5 \%$ & $5 \%$ & $6 \%$ & $3 x$ & $4 \%$ & $11 \%$ & $4 \%$ & $4 \%$ & $4 \%$ & $5 \%$ & $18 \%$ \\
\hline \multicolumn{20}{|l|}{ Section D } \\
\hline \begin{tabular}{|l} 
Sumberion \\
Number
\end{tabular} & 10 & 10 & 10 & 10 & 9 & 10 & 5 & 0 & 10 & 10 & 10 & 10 & 10 & 10 & 10 & 10 & 10 & 10 & 10 \\
\hline Avg. Std. Diff. & $6 \%$ & $9 \%$ & $6 \%$ & 4x & $8 \%$ & $7 \%$ & $5 \%$ & & $10 \%$ & $11 \%$ & $13 \%$ & 9\% & $14 \%$ & $20 \%$ & $10 \%$ & $10 \%$ & $8 \%$ & $13 \%$ & $22 \%$ \\
\hline Avg. Std. Dev. & $4 \%$ & $6 \%$ & $4 \%$ & $3 \%$ & $5 \%$ & $5 \%$ & $3 \%$ & & $7 \%$ & $8 \%$ & $9 \%$ & $6 \%$ & $10 \%$ & $14 \%$ & $7 \%$ & $7 \%$ & $6 \%$ & $9 \%$ & $16 \%$ \\
\hline
\end{tabular}




\begin{tabular}{|c|c|c|c|c|c|c|c|c|c|c|c|c|c|c|c|c|c|c|}
\hline $\begin{array}{l}\text { ACCURACY-Standard } \\
\text { Reference SARL }\end{array}$ & $\begin{array}{l}\text { Ag, ppm, } \\
\text { ICP }-40\end{array}$ & $\begin{array}{l}\text { As, ppm, } \\
\text { ICP }-40\end{array}$ & $\begin{array}{l}\text { Aw, ppen, } \\
K C P-40\end{array}$ & $\begin{array}{l}\text { Ba, ppom, } \\
\text { ICP }-40\end{array}$ & $\begin{array}{l}\text { Be, ppm, } \\
\mathrm{ICP}-40\end{array}$ & $\begin{array}{l}B i, p p m, \\
1 C P-40\end{array}$ & $\begin{array}{l}C d, p p m, \\
\text { ICP }-40\end{array}$ & $\begin{array}{l}\text { Ce, ppm, } \\
\angle C P-40\end{array}$ & $\begin{array}{l}\text { Co, ppm, } \\
\text { ICP }-40\end{array}$ & $\begin{array}{c}C_{r, p p m,} \\
\text { ICP-40 }\end{array}$ & $\begin{array}{l}\text { Cu, ppm, } \\
\text { ICP }-40\end{array}$ & $\begin{array}{l}\text { Eu, ppm, } \\
\text { KCP }-40\end{array}$ & $\begin{array}{l}\text { Ga, Ppm, } \\
\text { ICP }-40\end{array}$ & $\begin{array}{l}\text { Ho, ppm, } \\
\text { ICP }-40\end{array}$ & $\begin{array}{l}\text { La, ppm, } \\
\text { ICP }-40\end{array}$ & $\begin{array}{c}\text { LI, ppm, ICP } \\
40\end{array}$ & $\begin{array}{l}M_{n, p p m,} \\
M C P-40\end{array}$ & $\begin{array}{l}\text { Mo, ppm, } \\
\text { ICP }-40\end{array}$ \\
\hline analyzed as & 3 & 22 & $<B$ & 937 & 4 & $<50$ & 3 & 155 & 8 & 65 & 358 & $<2$ & 14 & $<4$ & 74 & 25 & 2080 & 14 \\
\hline anatyzed as & 3 & 19 & $<8$ & 919 & 5 & $<50$ & 2 & 161 & 9 & 108 & 340 & $<2$ & 14 & $<4$ & 78 & 25 & 2140 & 13 \\
\hline anatyzed as & 3 & 25 & $<8$ & 916 & 4 & $<50$ & 3 & 156 & 7 & 38 & 353 & $<2$ & 19 & $<4$ & 76 & 25 & 1970 & 14 \\
\hline Average & 3 & 22 & & 924 & 4 & & 3 & 157 & 8 & 70 & 350 & & 16 & & 76 & 25 & 2063 & 14 \\
\hline Accepted Value & 2.6 & 16.5 & 0.325 & 879 & 3.2 & 1.1 & 2.5 & 150 & 7.5 & 110 & 370 & 1.5 & 17 & 1.9 & 75 & 28 & 2094 & 13 \\
\hline Rel. Std. Diff. & $17 \%$ & $33 \%$ & & $5 \%$ & $34 \%$ & & $7 \%$ & $5 \%$ & $7 \%$ & $-36 \%$ & $-5 \%$ & & $-8 \%$ & & $1 \%$ & $-11 \%$ & $-1 \%$ & $5 \%$ \\
\hline \multicolumn{19}{|l|}{$\begin{array}{l}\text { Standard Reference } \\
\text { SARM }\end{array}$} \\
\hline anelyzed as & 4 & 38 & $<8$ & 793 & 2 & $<50$ & 5 & 111 & 11 & 70 & 299 & $<2$ & 10 & $<4$ & 53 & 25 & 5230 & 12 \\
\hline analyzed as & 5 & 36 & $<8$ & 801 & $\frac{2}{2}$ & $<50$ & 6 & 122 & 11 & 75 & 303 & $<2$ & 13 & $<4$ & 59 & 26 & 5140 & 12 \\
\hline analyzed as & 5 & 42 & $<8$ & 816 & 2 & $<50$ & 5 & 111 & 12 & 64 & 298 & $<2$ & 9 & $<4$ & 55 & 26 & 5230 & 13 \\
\hline Average & 5 & 39 & & 803 & 2 & & 5 & 115 & 11 & 70 & 300 & & 11 & & 56 & 26 & 5200 & 12 \\
\hline Accepted Value & 3.1 & 37 & 0.345 & 764 & 2.4 & 1.33 & 4.76 & 120 & 11 & 101 & 320 & 0.67 & 20 & 1.72 & 61 & 30 & 5200 & 12 \\
\hline Rel. Std. Diff. & $51 \%$ & $5 \%$ & & $5 \%$ & $-17 \%$ & & $12 \%$ & $4 \%$ & $3 \%$ & $-31 \%$ & $-6 x$ & & $-47 \%$ & & $-9 \%$ & $-14 \%$ & $0 \%$ & $3 \%$ \\
\hline \multicolumn{19}{|l|}{$\begin{array}{l}\text { P8v-1 Bone Valley } \\
\text { Phosphorite, split } 16\end{array}$} \\
\hline analyzed as & $<2$ & $<10$ & $<8$ & 84 & 2 & $<10$ & 3 & 150 & 6 & 70 & 11 & 5 & $<4$ & 5 & 112 & 3 & 281 & 9 \\
\hline analyzed as & $<2$ & $<10$ & $<8$ & 85 & 2 & $<50$ & 3 & 162 & 6 & 90 & 7 & 5 & $<4$ & 6 & 119 & 3 & 319 & 9 \\
\hline Average & & & & 84.5 & 2 & & 3 & 156 & 6 & 80 & 9 & 5 & & 5.5 & 115.5 & 3 & 300 & 9 \\
\hline Rel. Std. Dev. & & & & $1 \%$ & $0 \%$ & & $0 \%$ & $5 \%$ & $0 \%$ & $18 \%$ & $31 \%$ & $0 \%$ & & $13 \%$ & $4 \%$ & $0 \%$ & $9 \%$ & $0 \%$ \\
\hline \multicolumn{19}{|l|}{ Project Standards, no } \\
\hline POWN-1 & $<2$ & 15 & $<8$ & 176 & $<1$ & $<50$ & 11 & 19 & 5 & 95 & 54 & 2 & 5 & $<4$ & 71 & 17 & 33 & 22 \\
\hline POW-2 & 8 & 23 & $<8$ & 246 & $<1$ & $<50$ & 51 & 44 & 3 & 1160 & 101 & 3 & 11 & $<4$ & 123 & 18 & 66 & 31 \\
\hline $\mathrm{POO}-1$ & $<2$ & 23 & $<8$ & 268 & 1 & $<50$ & 9 & 55 & 12 & 163 & 43 & 3 & 19 & $<4$ & 66 & 25 & 208 & 18 \\
\hline \multicolumn{19}{|l|}{$\begin{array}{l}\text { PRECISION- duplicates } \\
\text { anatyses }\end{array}$} \\
\hline \multicolumn{19}{|l|}{ Section C } \\
\hline \begin{tabular}{|l} 
Number \\
\end{tabular} & 2 & 0 & 0 & 6 & 2 & 0 & 6 & 6 & 2 & 6 & 6 & 5 & 0 & 2 & 6 & 6 & 5 & 6 \\
\hline Avg. Std. Diff. & $5 \%$ & & & $4 \%$ & $0 \%$ & & $1 \%$ & $6 \%$ & $11 \%$ & $30 \%$ & $6 \%$ & $8 \%$ & & $20 \%$ & $3 \%$ & $7 \%$ & $16 \%$ & $11 \%$ \\
\hline Avg. Std. Dev. & $3 \%$ & & & $3 \%$ & $0 \%$ & & $1 \%$ & $4 \%$ & $8 \%$ & $21 \%$ & $4 \%$ & $6 \%$ & & $14 \%$ & $2 \%$ & $5 \%$ & $11 \%$ & $8 \%$ \\
\hline \multicolumn{19}{|l|}{ Section D } \\
\hline \begin{tabular}{|l|} 
Number \\
\end{tabular} & 6 & 8 & 0 & 10 & 5 & 0 & 9 & 9 & 9 & 10 & 10 & 7 & 7 & 4 & 10 & 8 & 10 & 10 \\
\hline Avg. Std. Diff. & $18 \%$ & $11 \%$ & & $8 \%$ & $13 \%$ & & $11 \%$ & $13 \%$ & $13 \%$ & $31 \%$ & $8 \%$ & $17 \%$ & $9 \%$ & $23 \%$ & $10 \%$ & $7 \%$ & $14 \%$ & $12 \%$ \\
\hline Avg. Std. Dev. & $13 \%$ & $8 \%$ & & $5 \%$ & $9 \%$ & & $8 \%$ & $9 \%$ & $9 \%$ & $22 \%$ & $6 \%$ & $12 \%$ & $7 \%$ & $16 \%$ & $7 \%$ & $5 \%$ & $10 \%$ & $9 \%$ \\
\hline
\end{tabular}




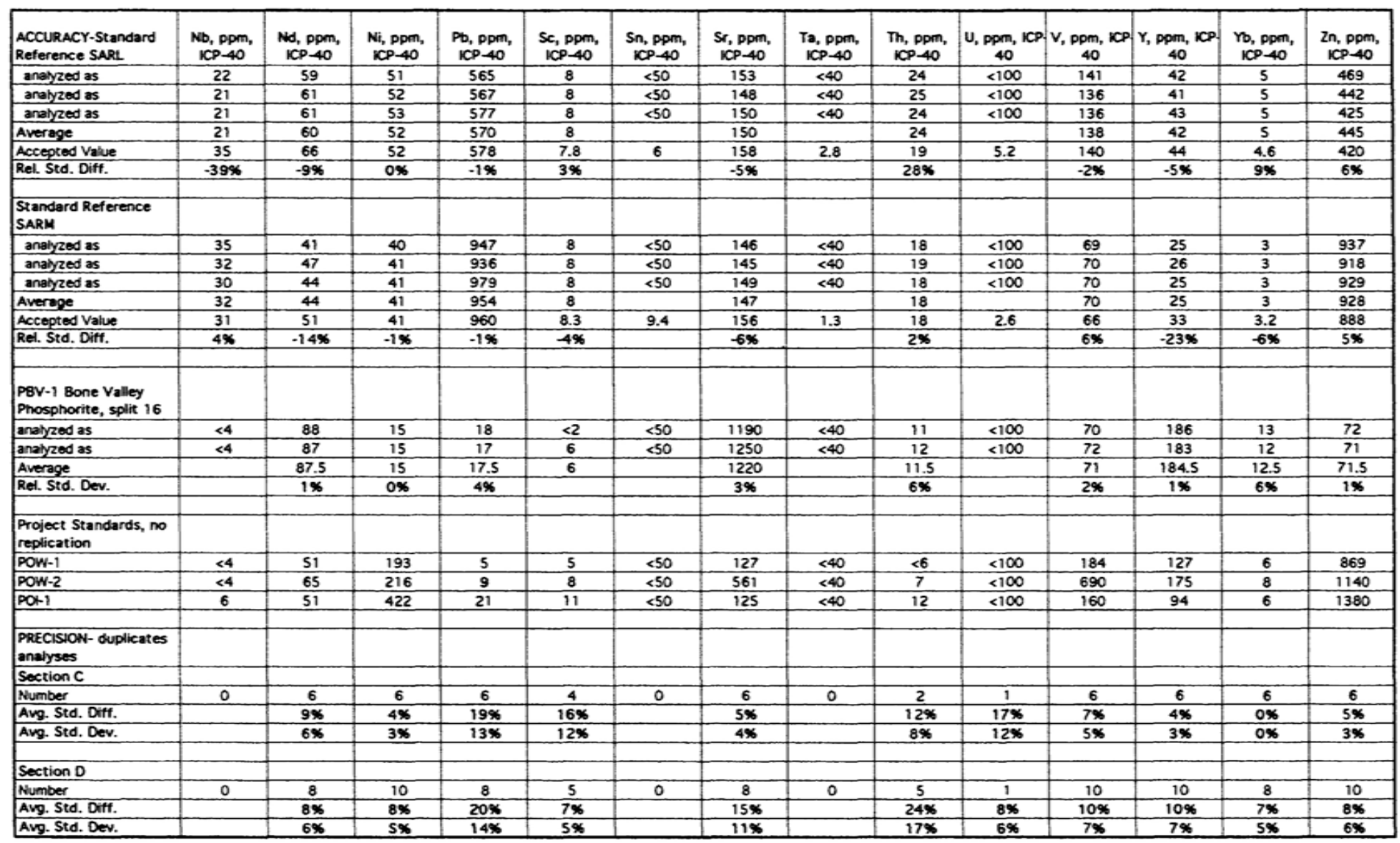

